2009-08-12

\title{
Analysis of Induced Vibrations in Fully-Developed Turbulent Pipe Flow Using a Coupled LES and FEA Approach
}

Thomas P. Shurtz

Brigham Young University - Provo

Follow this and additional works at: https://scholarsarchive.byu.edu/etd

Part of the Mechanical Engineering Commons

\section{BYU ScholarsArchive Citation}

Shurtz, Thomas P., "Analysis of Induced Vibrations in Fully-Developed Turbulent Pipe Flow Using a Coupled LES and FEA Approach" (2009). Theses and Dissertations. 1907.

https://scholarsarchive.byu.edu/etd/1907

This Thesis is brought to you for free and open access by BYU ScholarsArchive. It has been accepted for inclusion in Theses and Dissertations by an authorized administrator of BYU ScholarsArchive. For more information, please contact scholarsarchive@byu.edu, ellen_amatangelo@byu.edu. 


\title{
ANALYSIS OF INDUCED VIBRATIONS IN FULLY-DEVELOPED \\ TURBULENT PIPE FLOW USING A COUPLED \\ LES AND FEA APPROACH
}

by

Thomas P. Shurtz

\author{
A thesis submitted to the faculty of \\ Brigham Young University \\ in partial fulfillment of the requirements for the degree of
}

Master of Science

Department of Mechanical Engineering

Brigham Young University

December 2009 

Copyright (C 2009 Thomas P. Shurtz

All Rights Reserved 

BRIGHAM YOUNG UNIVERSITY

GRADUATE COMMITTEE APPROVAL

of a thesis submitted by

Thomas P. Shurtz

This thesis has been read by each member of the following graduate committee and by majority vote has been found to be satisfactory.

Date

Date

Date
R. Daniel Maynes, Chair

Jonathan D. Blotter

Steven E. Gorrell 



\section{BRIGHAM YOUNG UNIVERSITY}

As chair of the candidate's graduate committee, I have read the thesis of Thomas P. Shurtz in its final form and have found that (1) its format, citations, and bibliographical style are consistent and acceptable and fulfill university and department style requirements; (2) its illustrative materials including figures, tables, and charts are in place; and (3) the final manuscript is satisfactory to the graduate committee and is ready for submission to the university library.

Date

Accepted for the Department

\author{
R. Daniel Maynes \\ Chair, Graduate Committee
}

Larry L. Howell

Graduate Coordinator

Accepted for the College

Alan R. Parkinson
Dean, Ira A. Fulton College of Engineering
and Technology





\begin{abstract}
ANALYSIS OF INDUCED VIBRATIONS IN FULLY-DEVELOPED

TURBULENT PIPE FLOW USING A COUPLED

LES AND FEA APPROACH
\end{abstract}

Thomas P. Shurtz

Department of Mechanical Engineering

Master of Science

Turbulent flow induced pipe vibration is a phenomenon that has been observed but not fully characterized. This thesis presents research involving numerical simulations that have been used to characterize pipe vibration resulting from fully developed turbulent flow. The vibration levels as indicated by: pipe surface displacement, velocity, and acceleration are characterized in terms of the parameters that exert influence. The influences of geometric and material properties of the pipe are investigated for pipe thickness in the range 1 to $8 \mathrm{~mm}$ at a diameter of $0.1015 \mathrm{~m}$. The effects of pipe elastic modulus are explored from 3 to $200 \mathrm{GPa}$. The range of pipe densities investigated is 3,000 to $12,000 \mathrm{~kg} / \mathrm{m}^{3}$. All pipe parameters are varied for both a short pipe (length to diameter ratio $=3$ ) and a long pipe (length to diameter ratio $=24$ ). Further, the effects of 

varying flow velocity, fluid density and fluid viscosity are also explored for Reynolds numbers ranging from $9.1 \times 10^{4}$ to $1.14 \times 10^{6}$. A large eddy simulation fluid model has been coupled with a finite element structural model to simulate the fluid structure interaction using both one-way and two-way coupled techniques. The results indicate a strong, nearly quadratic dependence of pipe wall acceleration on average fluid velocity. This relationship has also been verified in experimental investigations of pipe vibration. The results also indicate the pipe wall acceleration is inversely dependant on wall thickness and has a power-law type dependence on several other variables. The short pipe and long pipe models exhibit fundamentally different behavior. The short pipe is not sensitive to dynamic effects and responds primarily through shell modes of vibration. The long pipe is influenced by dynamic effects and responds through bending modes. Dependencies on the investigated variables have been non-dimensionalized and assembled to develop a functional relationship that characterizes turbulence induced pipe vibration in terms of the relevant parameters. The functional relationships are presented for both the long and short pipe models. The functional relationships can be used in applications including non-intrusive flow measurement techniques. These findings also have applications in developing design tools in pipe systems where vibration is a problem. 



\section{ACKNOWLEDGMENTS}

I would like to express thanks to Dr. Daniel Maynes for his continued guidance throughout my research. His insight and encouragement have been invaluable in writing this thesis and completing a project that I have thoroughly enjoyed. He has not only been a wonderful advisor, but a great teacher as well. It was my experience with him in the classroom that convinced me to pursue a project where I would be working alongside him.

I would additionally like to thank Dr. Jonathan Blotter for his significant input and suggestions and the hours he has spent through our research meetings aiding my understanding of the project.

This project has been generously funded by both Control Components, Inc. and Genscape, Inc. Their interest in this project has not only made this part of my education possible, but has allowed the discovery of the results presented in this thesis, and will continue to improve our understanding of flow induced vibration.

Finally, I need to thank my family for their patience and trust during my involvement with this project. I would especially like to thank my wife for her support and willingness to put up with having a graduate student for a husband. Her love and encouragement have been my primary motivation for completing this thesis. 



\section{TABLE OF CONTENTS}

LIST OF TABLES .....................................................................................................ii

LIST OF FIGURES ............................................................................................................. $\mathrm{xV}$

1 Introduction........................................................................................................................... 1

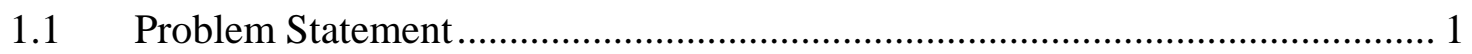

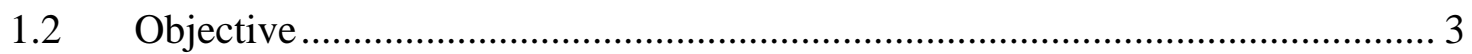

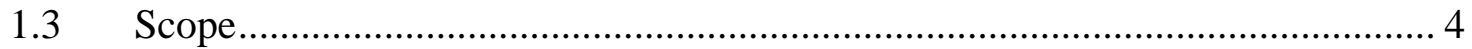

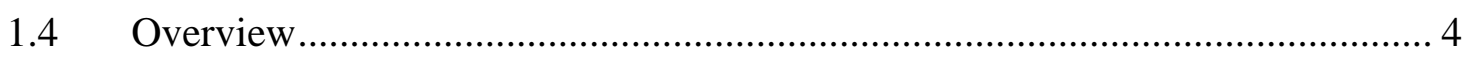

2 Background ...................................................................................................................... 7

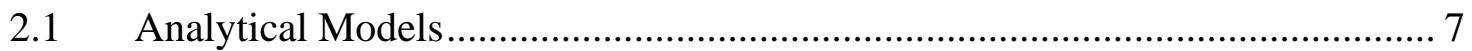

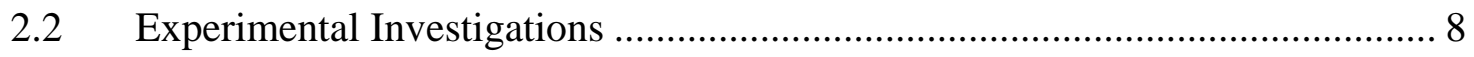

$2.3 \quad$ Numerical Approaches ……………………................................................ 10

$2.4 \quad$ Research Contribution .................................................................................... 12

$3 \quad$ LES Fluid Model ......................................................................................................... 15

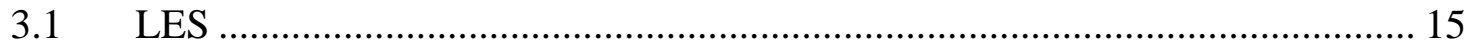

3.1.1 Governing Equations of Fluid Flow ……………………......................... 16

3.1.2 Smagorinsky Model ................................................................................... 18

$3.2 \quad$ Flow Physics ......................................................................................... 19

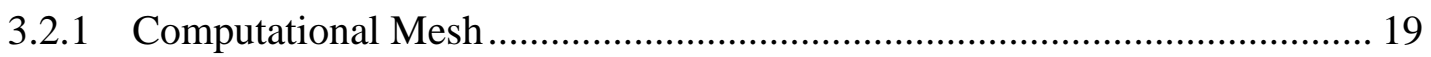

3.2.2 Boundary and Initial Conditions ................................................................ 23

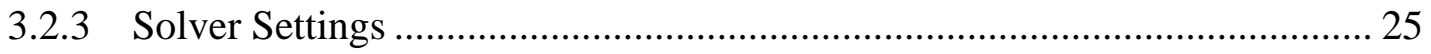

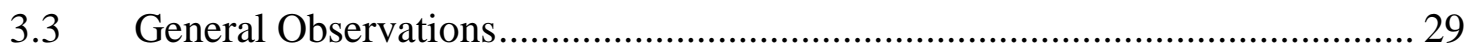

3.4 Model Verification.................................................................................... 29

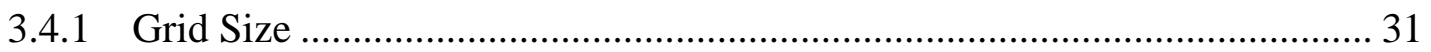

3.4.2 Time Step ………………………........................................................ 34 


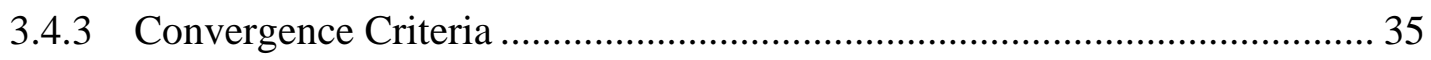

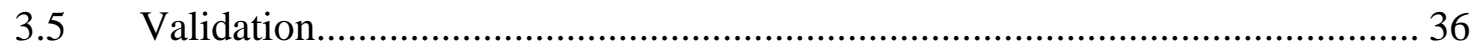

$4 \quad$ FEA Structural Model..................................................................................................... 41

4.1 Model Physics.................................................................................................... 42

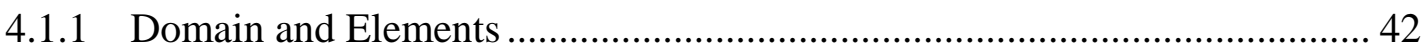

4.1.2 Boundary Conditions .............................................................................. 44

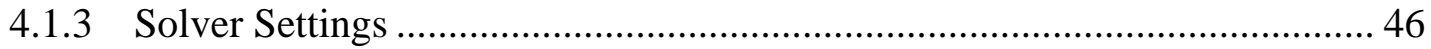

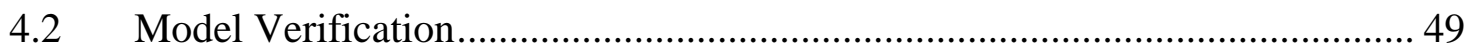

4.2.1 Element Type …………………………………...................................... 49

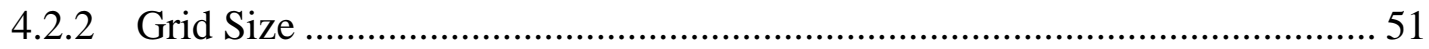

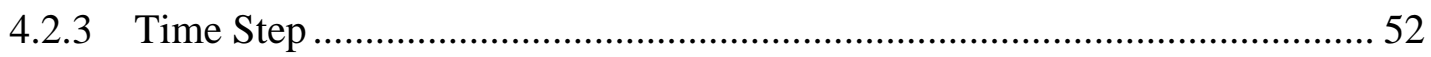

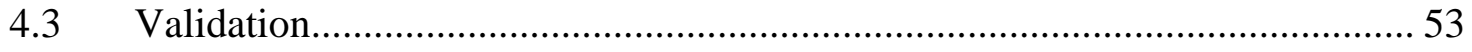

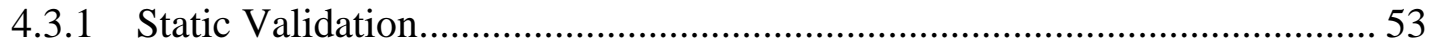

4.3.2 Dynamic Validation ............................................................................. 55

$5 \quad$ FSI Coupling Procedure............................................................................................... 59

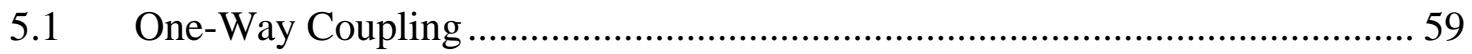

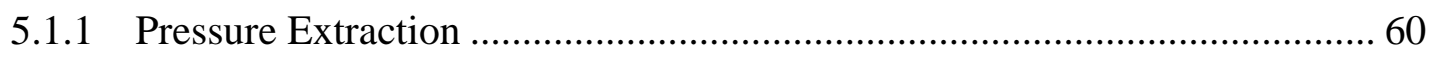

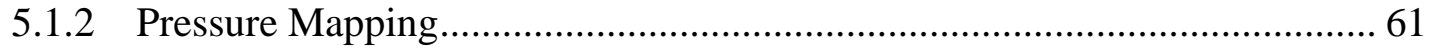

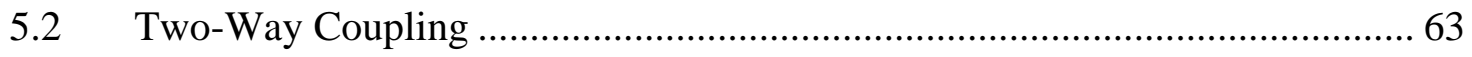

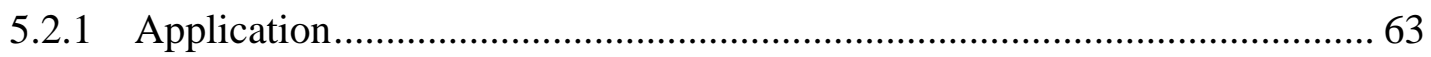

5.2.2 Comparison With One-Way Approach............................................................ 64

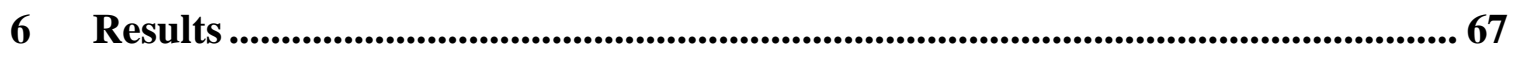

6.1 General Behavior .......................................................................................... 67

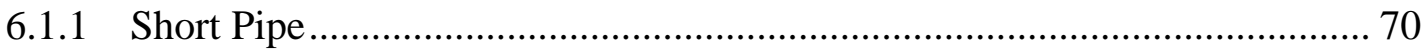

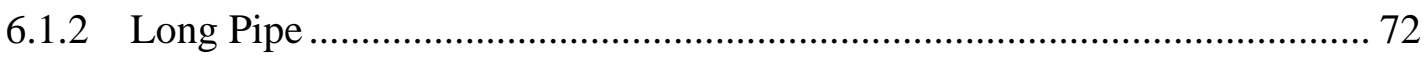

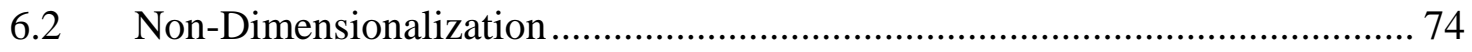

6.3 Specific Variable Effects ............................................................................. 76

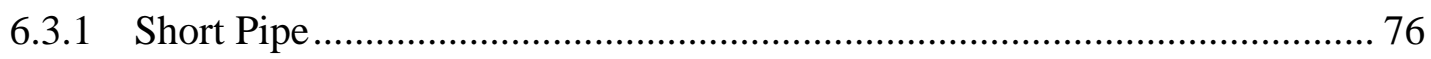

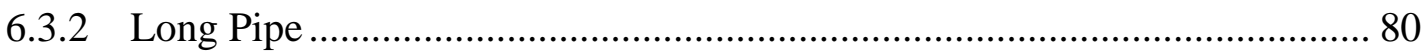

6.4 Complete Functional Relationships ................................................................. 86

6.5 Comparison to Experiments.......................................................................... 91 


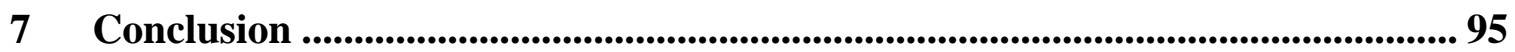

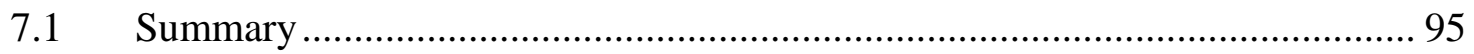

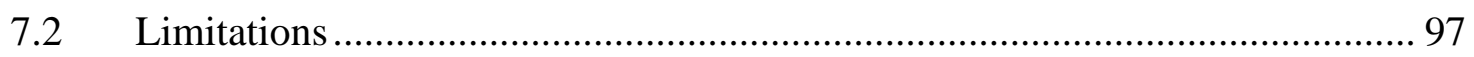

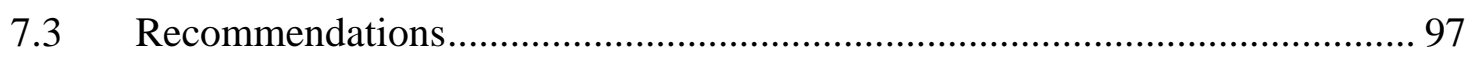

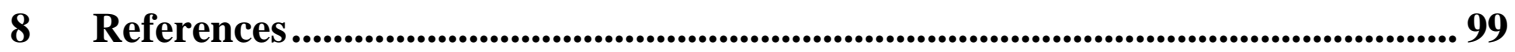

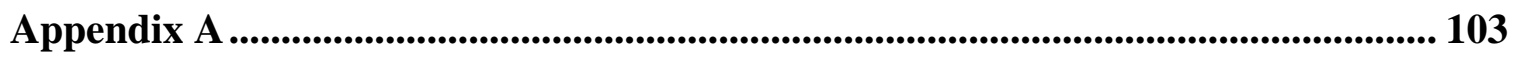

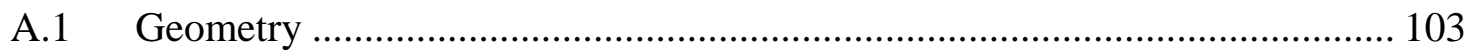

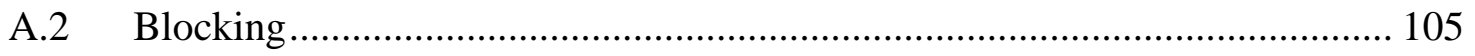

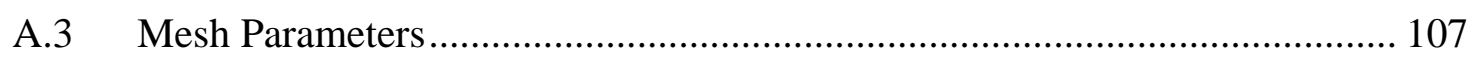

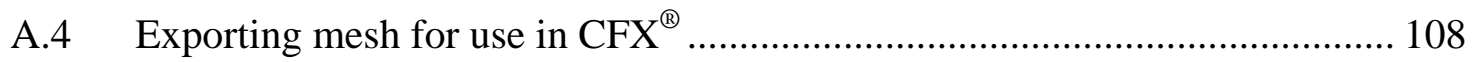

Appendix B ................................................................................................................... 109

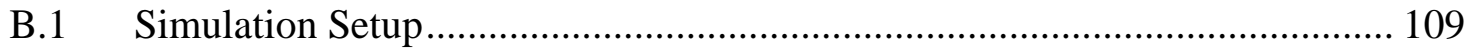

B.2 Boundary Conditions ........................................................................... 110

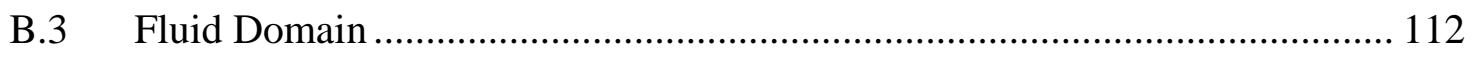

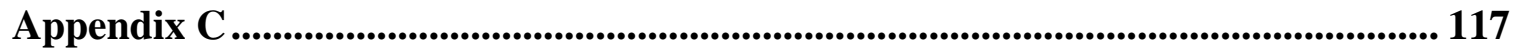

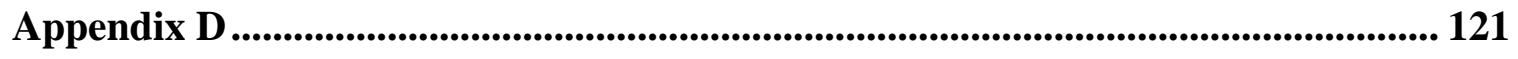

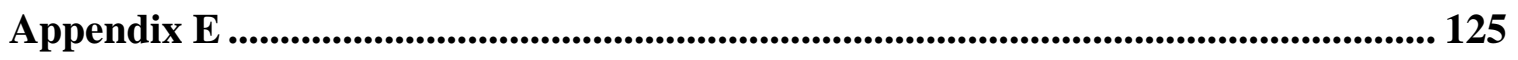

Appendix F ..................................................................................................................... 129 


\section{LIST OF TABLES}

Table 1-1 Values of variables explored........................................................................ 5

Table 3-1 Dimensionless grid spacing at wall.....................................................23

Table 3-2 Solver settings used for all simulations ..................................................26

Table 3-3 Settings used to vary Reynolds number .................................................29

Table 3-4 Wall shear stress for different meshes ........................................................33

Table 3-5 Pressure fluctuation amplitude for different meshes ....................................34

Table 3-6 Friction factor comparison with experimental data .....................................39

Table 4-1 Structural mesh refinement effects ......................................................51

Table 4-2 Structural model static validation summary ................................................55

Table 5-1 Coupling technique comparison summary ................................................66

Table 6-1 Dimensionless dependant variables ..........................................................74

Table 6-2 Dimensionless independent variables .......................................................75

Table 6-3 Summary of first order effects of all variables ...........................................86

Table 6-4 Thompson experiment pipe geometry ...................................................93

Table 7-1 Functional relationships: Result summary ...............................................96 


\section{LIST OF FIGURES}

Figure 3-1 End view of fluid mesh ...............................................................21

Figure 3-2 Contours of fluid velocity at first and last time step of $4 \mathrm{~m} / \mathrm{s}$ solution............30

Figure 3-3 Meshes used for verification ...............................................................31

Figure 3-4 Grid resolution comparison: Time-average velocity profile from $0.8 \mathrm{~m} / \mathrm{s}$

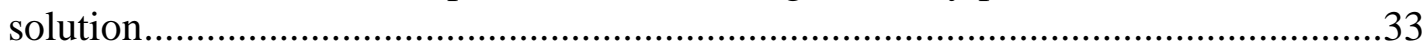

Figure 3-5 Time step size comparison: Time-average velocity profile from $0.8 \mathrm{~m} / \mathrm{s}$

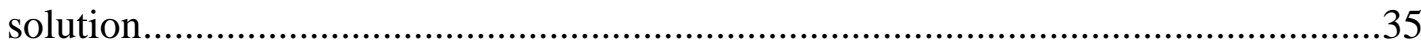

Figure 3-6 High Reynolds number velocity profile................................................38

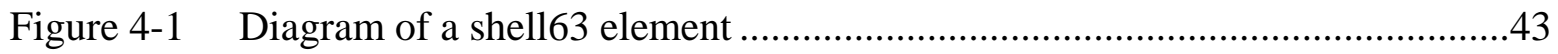

Figure 4-2 Short pipe with boundary conditions applied ...........................................45

Figure 4-3 Long pipe with boundary conditions applied..........................................46

Figure 4-4 Relative difference in the standard deviation of the wall displacement between shell vs. solid element modeling as a function of wall thickness ................50

Figure 4-5 Effect of time step size on pipe wall acceleration.......................................53

Figure 4-6 Comparison of ANSYS and experimental natural frequencies (lighter bands indicate uncertainty range in ANSYS predictions) ...............................................57

Figure 5-1 Pressure field before and after averaging (original field on left)..................62

Figure 5-2 Coupling technique comparison: Time series displacement........................65

Figure 6-1 $\quad P^{\prime}$ and $\tau_{\mathrm{w}}$ as functions of average fluid velocity in a pipe having $D=0.1015$ $\mathrm{m}$ with water as the working fluid. ....................................................................68

Figure 6-2 Wall pressure fluctuations as a function of frequency for the $10 \mathrm{~m} / \mathrm{s}$ flow solution.

Figure 6-3 Short pipe pressure and wall displacement as a function of frequency for $U$ $=10 \mathrm{~m} / \mathrm{s}, D=0.1015 \mathrm{~m}, t=3 \mathrm{~mm}, E=3.7 \mathrm{GPa}$ 
Figure 6-4 Short pipe surface deflection pattern for $U=10 \mathrm{~m} / \mathrm{s}, D=0.1015 \mathrm{~m}, t=$ $3 \mathrm{~mm}, E=3.7 \mathrm{GPa}$

Figure 6-5 Long pipe pressure and wall displacement as a function of frequency content for $U=10 \mathrm{~m} / \mathrm{s}, D=0.1015 \mathrm{~m}, t=2 \mathrm{~mm}, E=21 \mathrm{GPa}, \rho_{\mathrm{eq}}=3000 \mathrm{~kg} / \mathrm{m}^{3}, \beta$ $=0.001$. Also shown are pipe natural frequencies predicted using a modal analysis.

Figure 6-6 Long pipe wall displacement pattern for $U=10 \mathrm{~m} / \mathrm{s}, D=0.1015 \mathrm{~m}, t=2$ $\mathrm{mm}, E=21 \mathrm{GPa}, \rho_{\mathrm{eq}}=3000 \mathrm{~kg} / \mathrm{m}^{3}, \beta=0.001$

Figure 6-7 $\delta^{*}, V^{*}$, and $A^{*}$ as functions of Reynolds number for the short pipe model with $t^{*}=0.0296$ and $E^{*}=3.71 \times 10^{6}$......

Figure 6-8 $\delta^{*}, V^{*}$, and $A^{*}$ as functions of $t^{*}$ for the short pipe model with $R e_{\mathrm{D}}=1.14 \times 10^{5}$ and $E^{*}=3.71 \times 10^{6}$

Figure 6-9 $\delta^{*}, V^{*}$, and $A^{*}$ as functions of $E^{*}$ for the short pipe model with $R e_{\mathrm{D}}=$ $4.55 \times 10^{5}$ and $t^{*}=0.0296$.

Figure 6-10 $\delta^{*}, V^{*}$, and $A^{*}$ as functions of $R e_{\mathrm{D}}$ for the long pipe model with $t^{*}=0.0197$, $L^{*}=23.6, \rho^{*}=3.01, \omega^{*}=0.473$, and $\zeta=0.047$

Figure 6-11 $\delta^{*}, V^{*}$, and $A^{*}$ as functions of $t^{*}$ for the long pipe model with $R e_{\mathrm{D}}=$ $1.14 \times 10^{6}, L^{*}=23.6, \rho^{*}=10.0, \omega^{*}=0.473$, and $\zeta=0.047$

Figure 6-12 $\delta^{*}, V^{*}$, and $A^{*}$ as functions of $L^{*}$ for the long pipe model with $R e_{\mathrm{D}}=$ $1.14 \times 10^{6}, t^{*}=0.0197, \rho^{*}=3.01, \omega^{*}=0.473$, and $\zeta=0.047$

Figure 6-13 $\delta^{*}, V^{*}$, and $A^{*}$ as functions of $\rho^{*}$ for the long pipe model with $R e_{\mathrm{D}}=$ $1.14 \times 10^{6}, t^{*}=0.0296, L^{*}=23.6, \omega^{*}=0.473$, and $\zeta=0.047$.

Figure 6-14 $\delta^{*}, V^{*}$, and $A^{*}$ as functions of $\omega^{*}$ for the long pipe model with $R e_{\mathrm{D}}=$ $1.14 \times 10^{6}, t^{*}=0.0296, L^{*}=23.6, \rho^{*}=12.0$, and $\zeta=0.047$.

Figure 6-15 $\delta^{*}, V^{*}$, and $A^{*}$ as functions of $\zeta$ for the long pipe model with $R e_{\mathrm{D}}=1.14 \times 10^{6}$, $t^{*}=0.0197, L^{*}=23.6, \rho^{*}=3.01$, and $\omega^{*}=0.473$. 85

Figure 6-16 $\delta^{*}$ as a function of $R e_{\mathrm{D}}{ }^{-0.26} t^{*-2.05} E^{*-1.00}$ for all short pipe model results.............88

Figure 6-17 $V^{*}$ as a function of $R e_{\mathrm{D}}{ }^{-0.30} t^{*-1.96} E^{*-1.00}$ for all short pipe model results ............88

Figure 6-18 $A^{*}$ as a function of $R e_{\mathrm{D}}{ }^{-0.38} t^{*-0.90} E^{*-1.00}$ for all short pipe model results

Figure 6-19 $\delta^{*}$ as a function of $R e_{\mathrm{D}}{ }^{-0.20} t^{*-1.16} L^{*-0.72} \rho^{*-1.00} \omega^{*-1.81} \zeta^{0.22}$ for all long pipe model results 
Figure 6-20 $V^{*}$ as a function of $\operatorname{Re}_{\mathrm{D}}{ }^{-0.12} t^{*-1.06} L^{*-0.34} \rho^{*-1.00} \omega^{*-0.67} \zeta^{-0.51}$ for all long pipe model results

Figure 6-21 $A^{*}$ as a function of $R e_{\mathrm{D}}{ }^{-0.18} t^{* 0.04} L^{*-0.16} \rho^{*-1.00} \omega^{*-0.10} \zeta^{-0.35}$ for all long pipe

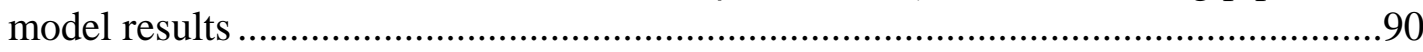

Figure 6-22 Comparison to Evans experimental data ...............................................92

Figure 6-23 Comparison to Thompson experimental data with linear fit.........................94

Figure A-1 Basic geometry showing subdivided curves ........................................... 105

Figure A-2 Block with edges associated to geometry .............................................. 106

Figure A-3 Resized block split into sections using O-grid......................................... 107

Figure A-4 Completed mesh as seen from the inlet end of the pipe ............................. 108 
xviii 


\section{Introduction}

\subsection{Problem Statement}

Flow induced pipe vibration is a phenomenon that is readily observed in almost any pipe system that involves fluid motion. There are several possible sources of unsteady loading on pipe surfaces. Some of these include vortex shedding, turbulence, cavitation, pulsating flow, and two-phase sloshing. The result of this unsteady loading is dynamic deformation of the pipe wall. This thesis focuses specifically on the topic of pipe vibration caused by turbulent fluid flow through the pipe. While this type of flow induced vibration is easily observed and has been the subject of some investigation and research, the phenomenon has not been well characterized. In industrial applications vibration can result in fatigue failure of pipe systems requiring costly maintenance and repairs [1]. With a more complete understanding of how pipe vibration is related to other variables, design tools for vibration resistant systems could be developed. Non-intrusive flow measurement techniques relying on vibration measurements could also be improved with a set of functional relationships tying vibration level and flow rate to other important variables [2].

Several of the variables that exert influence have been explored by other researchers and are described briefly in chapter 2 [3-13]. Analytical, experimental, and numerical 
research efforts in this field have been able to identify some basic relationships describing the dependence of pipe vibration level on flow parameters and pipe geometry such as average flow velocity and pipe diameter $[6,7,11]$. Each of the research approaches has inherent limitations and advantages, but none have resulted in a clear or complete picture or set of relations that describe pipe vibration in terms of the many influential parameters.

Analytical approaches allow precise functional relationships to be determined quickly, and do not require any expensive equipment. Most analytical models of fluid carrying pipes such as those introduced by Païdoussis [6] are limited to exploring average flow quantities only. Because of the complexity of the governing equations describing fluid flow, analytical solutions are impossible for all but the simplest cases. While the cylindrical geometry of pipes is simple enough for analytical solutions when the flow is restricted to the laminar regime, the unsteady and chaotic nature of turbulent flow renders the equations intractable. Analytical approaches tend to focus on mathematical representations of the pipe structure, and only account for average pressure and wall shear stress in the fluid.

Experimental efforts to create and measure turbulent flow induced vibrating pipe systems provide results with direct application to industry, but can be time consuming and expensive. They are also limited by the available pipe materials and fluids, making controlled variation of parameters difficult. It is also often impossible to isolate one particular variable without affecting others. Experimental systems are susceptible to vibration from other sources, such as pump or valve noise, or entire facility vibration 
from traffic or machinery. Previous results from experiments such as those performed by Evans [7] are valuable for comparison, but are certainly limited in coverage.

Numerical efforts at modeling a coupled system that includes time accurate turbulent fluid flow and dynamic pipe response have had some success, but have generally been limited by available computational power [11]. Limitations on processor

speed, system memory, and storage capacity have made numerical techniques useful only for simple geometries and low Reynolds numbers. While recent advances in computer technology have expanded the range of usefulness for these techniques, they have not yet been applied to developing a complete set of functional relationships that characterize pipe vibration caused by fully developed turbulent flow.

\subsection{Objective}

The objective of this work is to provide a more complete characterization of fully developed turbulent flow induced pipe vibration than currently exists. The functional relationships that characterize the phenomenon are determined using a numerical model coupling turbulent fluid flow with a dynamic pipe structure. The model uses a combination of large eddy simulation (LES) to solve for the time varying pressure field in the fluid domain, and finite element analysis (FEA) to solve for the transient structural response of the pipe. The effects of the most influential variables are explored by independently adjusting each. The final goal of the research is to assemble a set of functional relationships that can be used as a design tool, and with further application in improving non-intrusive flow measurement techniques. An additional objective is to develop a methodology which can be used for additional exploration of complex variable 
interactions so the functional relationships can continually be expanded and improved. Finally the resultant functional relationships will be applied to experimental data in an attempt to improve understanding of the physical interactions present in vibrating pipes.

\subsection{Scope}

The stated objectives will be reached by establishing a LES flow simulation in ANSYS $^{\circledR}$ CFX $^{\circledR}$ and obtaining flow solutions at seven different Reynolds numbers. The transient pressure on the wall will be used to excite the FEA structural model to explore the structural variables. Two classes of pipes characterized by different aspect ratios will be modeled. A short pipe having length to diameter ratio $(L / D)$ of 3 will be compared to a long pipe having $L / D=24$. Both the long pipe and the short pipe models will be used to explore the effects of pipe elastic modulus, pipe density, wall thickness, inside pipe diameter, and user specified damping ratio in the modeling scheme. The range of variables explored is shown in Table 1-1. A set of non-dimensional variables will be created that includes all of the explored variables listed in addition to the dependant variables $\delta^{\prime}, V^{\prime}$, and $A^{\prime}$, which indicate the standard deviation of the pipe wall deflection,

pipe wall speed, and pipe wall acceleration, respectively. A set of functional relationships for the non-dimensional variables will then be determined.

\subsection{Overview}

Chapter 2 of this thesis contains a review of the literature relevant to the topic of turbulent flow induced pipe vibration. Previous work and contributions from other 
researchers in this area are discussed to determine the current state of this field of research. Specific contributions and delimitations of this work are discussed in the

Table 1-1 Values of variables explored

\begin{tabular}{|c|c|c|c|}
\hline Variable & \multicolumn{2}{|c|}{ Values Explored } & Units \\
\hline $\begin{array}{c}\text { Pipe Elastic } \\
\text { Modulus }\end{array}$ & $2,3.7,10,20,40,70,200$ & $3.7,6,12,25,50,100,200$ & $\mathrm{GPa}$ \\
\hline Pipe Density & $1,1.3,2,3,4,5,6,7,8$ & $1,1.3,2,3,4,5,6,7,8$ & $1000 \mathrm{~kg} / \mathrm{m}^{3}$ \\
\hline $\begin{array}{c}\text { Pipe Wall } \\
\text { Thickness }\end{array}$ & $1,2,3,4,5,6,7,8$ & $1,2,3,4,5,6$ & $\mathrm{~mm}$ \\
\hline $\begin{array}{c}\text { Pipe Material } \\
\text { Damping }\end{array}$ & $0.01,0.05,0.1,0.5,1,5$ & $0.01,0.05,0.1,0.5,1,5$ & $\mathrm{~s}$ \\
\hline $\begin{array}{c}\text { Reynolds } \\
\text { Number }\end{array}$ & $0.9,1,2,3.4,4.6,6,8,11$ & $0.9,1,2,3.4,4.6,6,8,11$ & 100,000 \\
\hline
\end{tabular}

context of the existing literature. Chapter 3 presents the methodology used to model the fluid domain using LES, such that others may reproduce the author's work. The fluid model is verified and validated, such that its use for the intended purpose can be justified. Chapter 4 discusses the structural FEA model. Again, the method of generating the model is discussed along with verification and validation studies. Chapter 5 is devoted to an explanation of the procedures used to couple the fluid and structural models. Chapter 6 presents the results obtained from solving the coupled model using the full range of variables. The results are also compared to those obtained from experimental work. Chapter 7 details the conclusions drawn from the research and concludes the body of the thesis. Finally, the references used in this thesis are listed, and applicable appendices are included. 


\section{Background}

Turbulent flow induced pipe vibration as a special application of fluid structure interaction (FSI) has been the subject of some attention, particularly as increasing computing power makes numerical approaches possible. This phenomenon has been explored using analytical and experimental techniques as well. This chapter reviews the available literature summarizing previous research efforts aimed at improving understanding of flow induced pipe vibration.

\subsection{Analytical Models}

One approach to determining the effects of important variables on pipe vibration is to solve a mathematical model of the pipe structure analytically. This generally involves making simplifying assumptions to allow the equations representing the system of interest to be solved. Most work in this area has focused on either response to a flowing inviscid fluid, or response variation due to pre-stressing caused by internal pressure and wall shear. The first method assumes a moving, inviscid fluid that affects the propagation of waves along the pipe material. Païdoussis [6] gives the linear equations of motion for

a pipe represented as either a beam or a cylindrical shell. The beam model assumes plug flow for the fluid while the shell model uses inviscid potential flow theory. While both models have the interesting result that the pipe will be unstable and subject to vibration 
above a critical flow velocity, neither considers turbulent fluctuations as the source of vibration. Xu and Yang [14] explain that previous models are not accurate near the critical velocity which causes pipe instability. They then expand on this type of approach to allow it to be more effective when the fluid velocity approaches the critical value. They use an Euler-Bernoulli model pipe containing fluid and develop a non-linear equation of motion. They apply the Newtonian method and use an order of magnitude analysis to simplify the equations. The primary goal of their studies was to determine the relative magnitude of internal resonances and at which fluid velocities they are excited. Again, potential flow theory is used and turbulent flow is not considered.

Additional efforts by Bochkarev and Matveenko [10] examine the added effects of varying boundary conditions at the pipe ends. Vassilev and Djondjorov [5] added a foundation along the pipe to represent periodic non-rigid supports. They allow the foundation stiffness to vary to simulate different spacing or types of supports. Both models used by Bochkarev and Matveenko and Vassilev and Djondjorov became too complex to use a strictly analytic solution procedure and employ numerical techniques. Any attempts to include distributed loading such as one would expect for turbulent wall pressures have required numerical techniques as well. Further discussion of approaches including distributed loading will therefore be saved for the numerical techniques section of this chapter.

\subsection{Experimental Investigations}

Measuring pipe vibration experimentally does not require any simplifying assumptions. While no modeling is done, the experimenter is limited to the use of 
available fluids and pipe materials. Also, the difficulty of isolating the vibration to that caused by fully developed turbulence presents itself.

Early work by Weyers [8] was done to determine how acoustic noise radiated from a pipe was affected by average flow speed in the pipe. The pipe wall in this case was very thin and the only measurement taken on the pipe wall was with a pressure transducer. This was done to attempt to estimate the pressure fluctuations within the pipe. The estimated pressure fluctuations were found to scale approximately with mean centerline velocity squared $\left(U^{2}\right)$. The root mean square (RMS) of the externally radiated acoustic pressure was found to scale with $U^{2.5}$. Although these results were hypothesized to relate to pipe wall vibration, no direct vibration measurements were made. Saito, et al. made specific vibration measurements on the pipe wall [15]. They quantified pipe wall acceleration $\left(A_{R M S}\right)$ in terms of mean fluid velocity. The source of vibration was identified as being turbulence related, although it was not isolated as fully developed turbulence because of the presence of an orifice plate near the measurement location.

Similar work by Evans [7] was done to demine the feasibility of using pipe vibration levels as an indicator of flow rate through the pipe. His work was based on the premise that pipe wall fluctuations indicated by the standard deviation of acceleration $\left(A^{\prime}\right)$ would be proportional to the standard deviation of the pressure at the wall $\left(P^{\prime}\right)$. It was further assumed that the pressure fluctuations would in turn be a function of the flow rate, $Q$. Evans experiments indicated a nearly quadratic relationship between flow rate and $A^{\prime}$ where the proportionality is indicated as $A^{\prime} \propto Q^{2}$. Other investigations included the use of other pipe sizes and materials. Evans' work used two pipe diameters (0.076 m and $0.102 \mathrm{~m}$ nominal) and three pipe materials (PVC, aluminum, and stainless steel). 
Although he was able to show that both diameter and pipe material influence the pipe vibration level, it is difficult to determine any well defined functional relationship with so little data. In this case the working fluid was always water, and the flow rate was varied by controlling the speed of a pump. Evans indicates that the data was filtered to remove frequency content in the range likely to be caused by pump noise, but no explanation is given of how this was determined. Because of the inclusion of a pump, and the bends and other geometry changes in the experimental setup, the source of vibration was not isolated to that induced by fully developed turbulent flow.

Durant and Robert made direct measurements of pipe wall vibration using accelerometers [16] and a laser vibrometer [4]. In addition, he measured the wall pressure fluctuations of air flow through a pipe. The acoustic fluctuations due to pressure wave reflection were filtered out and the cross spectra were used to create a wall pressure model in terms of convection velocity and correlation length. In these studies the object was to measure spectra and acoustic radiation rather than vibration amplitude. Only a single pipe and working fluid were used.

\subsection{Numerical Approaches}

Many numerical approaches to characterizing flow induced pipe vibration have focused on solving the structural pipe model while including approximations for the flow field. The research of Seo, et al. involves the use of time variable velocity, but applies potential flow theory like the analytical approaches [17]. In their studies, the bulk velocity was allowed to vary harmonically, but did not have the local and chaotic fluctuations characteristic of turbulent flow. Numerical techniques were then applied to 
solve the equation for motion of the pipe. They were able to show that with a pulsating fluid, pipe instability and vibration can occur at velocities lower than the critical velocity indicated in the analytical studies done by those like Païdoussis [6].

Applying distributed loading to the surface of the structural pipe model allows local turbulent fluctuations to be accounted for. In these models the pipe surface is given a temporally and spatially varying pressure load. Durant, et al. used numerical methods to solve a model including a cross-spectral representative pressure field integrated over the pipe wall [4]. The cross spectra were generated using experimental pressure measurements and a Corcos model. The purpose of their work was to compare frequency content of radiated acoustic pressure with experiment. The numerical model showed agreement within a few decibels of experimental measurements across the frequencies of interest (300-3000 Hz). Four discrete fluid velocities were used, but no attempt to quantify the effect of velocity on vibration level was made. Birgersson et al. used a similar approach of modeling the pressure load spectrally, but apply a different type of numerical technique using spectral super elements for increased computational efficiency when solving for the pipe wall response [9]. Finnveden, et al. use the same approach as Durant, but use a modified Chase model for the turbulent pressure spectra. The Chase model gives better results at higher frequencies [18].

All of the numerical efforts mentioned thus far have relied on statistical models of the turbulent pressure as a forcing function for various numerical representations of the pipe structure. This approach makes two-way coupling of the pipe motion and pressure field impossible, but does allow for computational efficiency by avoiding numerical 
modeling of the fluid domain. Also, the focus of the researchers has been directed at frequency content and acoustic noise, not the pipe motion itself.

Recent development in the field of computational fluid dynamics has made it possible to resolve the most significant levels of turbulence through LES. A full LES model of the fluid domain was used in work by Pittard to investigate the effects of fluid velocity, pipe diameter and pipe material on the standard deviation of pipe wall acceleration [11]. His model was quite limited by computational resources available at the time. The flow domain length was restricted to just over one diameter and the maximum Reynolds number achieved was 420,000. The FEA model of the pipe could only be solved for a maximum of 50 time-steps due to a file size limitation imposed by the file system. This resulted in low temporal resolution. Even with these limitations the model was able to generate results that fit very well with experimental measurements. Pittard examined six fluid velocities in water giving Reynolds numbers in the range 80,000 - 420,000. He also looked at the influence of diameter for three pipe sizes from $0.038 \mathrm{~m}-0.102 \mathrm{~m}$. The effect of pipe modulus was considered for static deflection comparisons. Because the displacements were small enough to be in the linear material behavior range, the deflection was exactly inversely proportional to the modulus. Dynamic pipe behavior was not considered in the studies performed by Pittard.

\subsection{Research Contribution}

The work presented in this thesis uses an approach very similar to that taken by Pittard. Spatial and temporal variations in the fluid domain will be determined through the use of an LES fluid model. This allows the problem of interest to be studied instead 
of a simplified analytical model. The inherent limitations present in all experimental work are avoided through the use of a numerical model that allows arbitrary variation in parameters where the effects of fully developed turbulent flow can be examined directly without contamination by external sources. The model used in the research leading to this thesis examines a wider range of Reynolds numbers and adds additional important parameters not present in previous work outlined in the literature. A fully coupled twoway model is also used for comparison to determine if it improves or changes results. This work does use approximations for the small scales of turbulence due to the nature of the LES model. Computational resources are still a limiting factor for LES models, and only a simple cylindrical flow field is considered. Wall roughness effects are not included in the model, but are present in many industrial applications. Because of the ideal conditions under which the model is operated, many of the results may be difficult to validate with experiments, and application of the results to real world scenarios must be done with care. However, the results of this numerical model have been compared to experiments, and the comparison is presented in this thesis for validation of the model. 


\section{LES Fluid Model}

This Chapter will describe the method used to create and solve the LES model of the fluid domain. An explanation of LES computational fluid dynamics (CFD) modeling is included in addition to the particular application used in this research. The procedures are described in such a way that the reader can duplicate the construction of the model used to obtain the results presented in this thesis. The ANSYS CFX program was chosen because it not only allows the required LES turbulence model, but it allows direct twoway coupling with the ANSYS ${ }^{\circledR}$ Multiphysics $^{\text {TM }}$ structural FEA model [19]. ADINA ${ }^{\circledR}$ was another software package that was considered for use. It also allows full coupling of the fluid and solid domain using the finite element method (FEM) for both, but it does not allow the periodic boundary condition required in the fluid domain for proper LES modeling of pipe flow. The coupling approach is discussed in detail in a later chapter, but the CFD portion of the modeling is explained in this chapter. Fluid model verification and validation studies are also presented in this chapter.

\subsection{LES}

The use of LES as a turbulence model is based on the concept that turbulent flows contain a wide range of length and time scales. The phenomenon of turbulence can be described as being unsteady, three-dimensional, non-linear, and chaotic but not random. 
Spatially coherent structures that change and develop in time are often referred to as eddies. The largest eddies, having dynamic and geometric properties related to the mean fluid flow, contain more energy than the smallest eddies. The LES approach makes use of this fact by applying spatial filters to the governing equations to remove, and therefore model, the smallest eddies while the large eddies are numerically simulated. This approach requires less computation than direct numerical simulation (DNS) which does not filter the equations and resolves all scales of turbulence. LES does take more computation than the commonly used Reynolds averaged Navier-Stokes (RANS) turbulence models, but RANS models do not resolve the turbulent pressure fluctuations responsible for the pipe vibration of interest. Because the LES model provides the ability to resolve pressure fluctuations while allowing solutions on more modest computer hardware, it is the model of choice for this research.

\subsubsection{Governing Equations of Fluid Flow}

The governing equations for a constant property, incompressible fluid with no body forces consist of the continuity equation, represented using index notation as:

$$
\frac{\partial u_{i}}{\partial x_{i}}=0
$$

and the Navier-Stokes equations, also shown using index notation as:

$$
\rho\left(\frac{\partial u_{i}}{\partial t}+\frac{\partial u_{i} u_{j}}{\partial x_{j}}\right)=\mu \frac{\partial^{2} u_{i}}{\partial x_{j}^{2}}-\frac{\partial P}{\partial x_{i}}
$$

where $u$ is the velocity, $\rho$ is the fluid density, $\mu$ is the dynamic viscosity and $P$ is the static pressure. Large eddy simulation (LES) filters these governing equations and 
decomposes the flow variables into large scale (resolved) and a small scale (unresolved) parts. Any flow variable $y$ can be written as:

$$
y=\bar{y}+y^{\prime}
$$

where $\bar{y}$ is the large scale part and $y^{\prime}$ is the unresolved small scale part. The large scale part is defined through volume averaging as:

$$
\bar{y}\left(x_{i}, t\right) \equiv \oiiint G\left(x_{i}-x_{i}^{\prime}\right) y\left(x_{i}^{\prime}, t\right) d x_{i}^{\prime},
$$

where $G\left(x_{i}-x_{i}^{\prime}\right)$ is a filter function (a simple box filter is often used). After performing the volume averaging, the spatially filtered Navier-Stokes equations become:

$$
\rho\left(\frac{\partial \bar{u}_{i}}{\partial t}+\frac{\partial \overline{u_{i} u_{j}}}{\partial x_{j}}\right)=\mu \frac{\partial^{2} \bar{u}_{i}}{\partial x_{j}^{2}}-\frac{\partial \bar{P}}{\partial x_{i}} .
$$

The non linear term carries all of the complexity and can be expanded as follows:

$$
\begin{aligned}
\overline{u_{i} u_{j}} & =\overline{\left(\overline{u_{i}}+u_{i}^{\prime}\right)\left(\overline{u_{j}}+\overline{u_{j}^{\prime}}\right)} \\
& =\overline{\overline{u_{i}} \overline{u_{j}}}+\overline{\overline{u_{i} u_{j}}}+\overline{\overline{u_{j} u_{i}}}+\overline{u_{i}^{\prime} u_{j}^{\prime}} .
\end{aligned}
$$

Derivations of the RANS equations use similar nomenclature, but the over-bar variables here are spatial averages, not temporal averages. The second and third terms (cross terms) of (3-7) are identically zero after taking the derivative when averaging in time, but this is not true for the volume averaged quantities. Even though the filter functions used by most LES models do not remove these cross terms exactly, they are considered small and are thus neglected even when not zero. Introduction of the sub grid scale (SGS) stresses as:

$$
\tau_{i j}=\overrightarrow{u_{i}^{\prime} u_{j}}-\overline{\bar{u}_{i} \bar{u}_{j}}
$$

allows the Navier-Stokes equations to be rewritten in a form that looks just like the unfiltered equations, but with an extra term as shown in (3-9): 


$$
\rho\left(\frac{\partial \bar{u}_{i}}{\partial t}+\bar{u}_{j} \frac{\partial \bar{u}_{i}}{\partial x_{j}}\right)=\mu \frac{\partial^{2} \bar{u}_{i}}{\partial x_{j}^{2}}-\frac{\partial \bar{P}}{\partial x_{i}}+\frac{\partial \tau_{i j}}{\partial x_{j}}
$$

\subsubsection{Smagorinsky Model}

The Smagorinsky model [20] combines the assumption of small cross terms with a mixing-length based eddy viscosity model for the SGS stress tensor $\tau_{i j}$. It therefore assumes that the SGS stresses are proportional to the filtered strain rate tensor as shown:

$$
\tau_{i j}-\frac{1}{3} \tau_{k k} \delta_{i j}=-2 \frac{\mu_{S G S}}{\rho} \bar{S}_{i j}
$$

where $\delta_{i j}$ is the Kronecker delta, $\mu_{S G S}$ is the sub grid scale viscosity, and the strain rate tensor is:

$$
\bar{S}_{i j}=\frac{1}{2}\left(\frac{\partial \bar{u}_{i}}{\partial x_{j}}+\frac{\partial \bar{u}_{j}}{\partial x_{i}}\right)
$$

To close the equation, a model for the SGS viscosity $\mu_{S G S}$ is needed. Based on dimensional analysis, the SGS viscosity can be expressed as the product of density, a length scale, and a velocity scale:

$$
\mu_{S G S} \propto \rho \Delta q_{S G S}
$$

where $\Delta$ is the length scale of unresolved motion, and $q_{S G S}$ is the velocity of the unresolved motion. The length scale $\Delta$ is determined by the local grid size to be:

$$
\Delta=\sqrt[3]{V_{c e l l}}
$$

where $V_{\text {cell }}$ is the volume of the local grid cell. In the Smagorinsky model, based on an analogy to the Prandtl mixing length model, the velocity scale is related to the gradients of the filtered velocity through the length scale:

$$
q_{S G S}=\Delta|\bar{S}|=\Delta \sqrt{2 \bar{S}_{i j} \bar{S}_{i j}}
$$


This finally yields the Smagorinsky model for the SGS viscosity:

$$
\mu_{S G S}=\rho\left(\Delta C_{S}\right)^{2}|\bar{S}|
$$

where $C_{s}$ is called the Smagorinsky constant. It usually has values ranging between 0.065 and 0.25 depending on the geometry and type of flow. Accurately modeling the flow near walls requires an adjustment to be made to the turbulent viscosity so it can be forced to approach zero at the wall. This modification procedure is called wall damping. The turbulent viscosity can be damped using a combination of a mixing length minimum function, and a viscosity damping function:

$$
\mu_{S G S}=\min \left(\kappa \cdot y_{w a l l}, f_{u} \Delta C_{s}\right)^{2}|\bar{S}|
$$

where $\kappa$ is the von Karmen constant, usually taken as $0.41, y_{\text {wall }}$ is the distance to the wall, and $f_{u}$ is a damping function. The damping function is often a constant, and the default value of 1.0 is used in the simulations for this research.

\subsection{Flow Physics}

The CFD model requires the definition of a discretized geometry called the mesh, or grid, where the solution will be calculated. Boundary conditions must be specified, and flow properties defined. Finally the solution procedure must be specified and the flow field initialized. These steps constitute the defining of the flow physics and numerical scheme for the model.

\subsubsection{Computational Mesh}

For the model used in this research the domain is a cylinder $0.3 \mathrm{~m}$ long and $.1015 \mathrm{~m}$ in diameter representing a section of the interior of a pipe. Other researchers have found 
that pipe lengths of 4/3D [11], 5D [21], and $2 \pi \mathrm{D}$ [22], where $\mathrm{D}$ is the diameter, produce adequate results. The length of pipe chosen for these simulations is approximately 3D, or slightly shorter than that suggested by Eggels [21] but significantly longer than that used by Pittard [11]. Although Pittard justified the use of a shorter pipe, it was used primarily because of computational limitations that are no longer as restrictive, so the longer pipe is used here to increase the accuracy of the results. Although the geometry is cylindrical, a Cartesian coordinate system is used placing the axis of the cylinder on the x-axis and placing the inlet on the yz plane.

The domain was discretized, or meshed using ANSYS ${ }^{\circledR}$ ICEM CFD $^{\mathrm{TM}}$. This is a utility intended strictly for generating meshes to be used for computational fluid dynamics (CFD) simulations. CFX also has meshing capabilities, but it does not give the user as much control or flexibility. Specifically the ICEM program allows the use of the O-grid type mesh. An O-grid type mesh is used which has a uniform square central region but gradually deforms to become more radial and perpendicular to the wall in the near wall region. This gives superior characteristics for the cylindrical domain being represented. The wall region has a slight inflation layer in the radial direction to allow better resolution of the high velocity gradients there. The square central region is 60 by 60 nodes. The O-grid ring that transitions from the square central region to the cylindrical wall contains 30 radial nodes and 236 circumferential nodes. There are 180 nodes in the axial direction. A view of the mesh as seen from the axial direction is shown in Figure 3-1. This mesh contains a total of 1,879,920 nodes and 1,848,175 hexahedral elements. The mesh contains 42,480 nodes on the wall where pressure information can be extracted or passed to the FEA solver in the coupling procedure. 


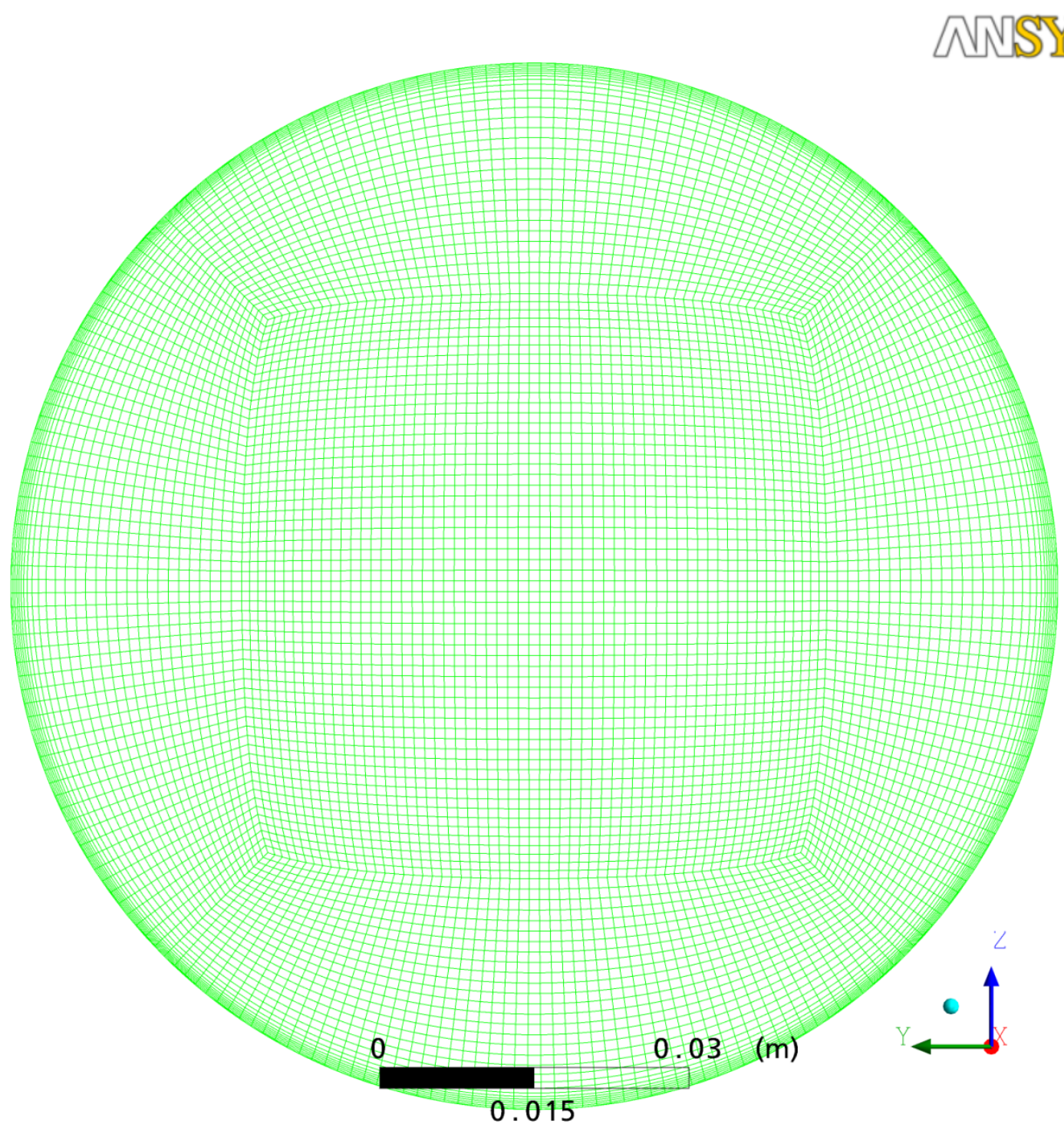

Figure 3-1 End view of fluid mesh

It is common to look at the mesh size in terms of dimensionless length scale parameters when determining how well the mesh can be expected to perform [23]. Generally, a mesh must meet minimum length scale requirements in order to achieve a given degree of accuracy. LES models require a fine mesh compared to other turbulence 
models, and therefore require shorter minimum length scales. The length scales are obtained by normalizing the grid spacing by the friction velocity and kinematic viscosity:

$$
\begin{aligned}
& r^{+}=(R-r) \frac{u_{\tau}}{v}, \\
& \Delta z^{+}=\Delta z \frac{u_{\tau}}{v}, \\
& R \Delta \theta^{+}=R \Delta \theta \frac{u_{\tau}}{v}
\end{aligned}
$$

where $R$ is the pipe inner radius, $r$ is the specific radial location of interest, $u_{\tau}$ is the friction velocity, $v$ is the kinematic viscosity, $\Delta z$ is the axial grid spacing, and $R \Delta \theta$ is the azimuthal grid spacing. The friction velocity is generally not known before the simulation is complete, but in this case there is significant experimental data that allows $u_{\tau}$ to be determined from the pipe friction factor through the following relation:

$$
u_{\tau}=U \sqrt{\frac{f}{8}}
$$

where the Darcy friction factor $f$ is available from experimental data on a Moody chart, or though some other empirical relationship. For the flows studied here the friction factor varies between about 0.018 at the lowest Reynolds number of $9.14 \times 10^{4}$, and 0.011 at the highest Reynolds number of $1.14 \times 10^{6}$. Using these values to convert the mesh spacing gives upper and lower dimensionless grid wall resolutions as shown in Table 3-1. Also shown is the dimensionless grid spacing used in studies by Pittard for comparison [11]. The present studies use slightly better dimensionless grid resolution in the near wall region than the resolution found to be acceptable by Pittard. 
Table 3-1 Dimensionless grid spacing at wall

\begin{tabular}{|c|c|c|c|c|c|c|}
\hline & $r^{+}$ & $z^{+}$ & $R \theta^{+}$ & $r / R$ & $\Delta z / L$ & $\Delta \theta / 2 \pi$ \\
\hline $\begin{array}{c}\text { Present Simulations } \\
R e_{\mathrm{D}}=1.14 \times 10^{6}\end{array}$ & 80 & 690 & 550 & 0.0038 & 0.0056 & 0.0042 \\
\hline $\begin{array}{c}\text { Present Simulations } \\
R e_{\mathrm{D}}=9.1 \times 10^{4}\end{array}$ & 8.5 & 70 & 57 & 0.0038 & 0.0056 & 0.0042 \\
\hline $\begin{array}{c}\text { Pittard } \\
R e_{\mathrm{D}}=4.15 \times 10^{5}\end{array}$ & 279 & 284 & 284 & 0.015 & 0.012 & 0.015 \\
\hline $\begin{array}{c}\text { Pittard } \\
R e_{\mathrm{D}}=8.3 \times 10^{4}\end{array}$ & 64 & 65 & 65 & 0.015 & 0.012 & 0.015 \\
\hline
\end{tabular}

Recommendations for the minimum values for LES models to be able to resolve a boundary layer are given by Piomelli [24]. He suggests using minimum values of $r^{+} \leq 1$, $z^{+} \approx 50-100$, and $R \theta^{+} \approx 15-40$. Rudman used slightly higher values but was still able to get results that compared well with experimental data [22]. The values for this mesh are too high to resolve the viscous sub-layer, but the near wall node is well into the log layer where most of the turbulent energy is producing fluctuations. Although the wall is not well resolved, the viscous sub-layer is not of particular interest when looking at the turbulent pressure fluctuations.

\subsubsection{Boundary and Initial Conditions}

The cylindrical surface of the domain is defined as a solid wall with the no-slip condition being enforced. Although options are available for including surface roughness effects, they are not used, and thus only smooth wall pipes are modeled. The no-slip wall boundary condition not only forces the fluid velocity to zero here, but also initiates the wall damping effects on the SGS viscosity. Also, because much of the boundary layer is 
not resolved, a wall modeling approach is applied. The wall model applies a synthetic law-of-the-wall velocity profile in the near wall region to adjust for the fact that the near wall node may be outside the viscous sub-layer. This results in velocity profiles that are not accurate in the lowest part of the log-law region or in the viscous sub-layer, but that produce better results in the regions which are resolved.

One of the difficulties in LES models is providing a proper time-varying velocity profile for use at the inlet. With no prior solution available, an inlet condition with specified velocity cannot be used unless the domain is long enough for the flow to develop and for instabilities to grow and become self-sustaining. For these simulations a periodic boundary is used to effectively connect the inlet and the outlet. The only value specified for this type of boundary is the total mass flow rate through the boundary. This condition forces the velocity and SGS properties to be matched at the inlet and outlet. The pressure will also be matched, but with an offset to account for viscous losses and to balance the drag force on the walls. The periodic condition can be applied most easily when the grid at both sides of the interface is identical as is true with the grid used here. This allows for 1:1 matching of values at each of the nodes. When the grids are different, interpolation techniques need to be used across the periodic boundary which results in additional computation time and additional error. The periodic boundary will let the flow develop as it effectively reenters the inlet each time it has passed through the entire domain.

Because LES models are by definition unsteady, proper initial conditions must be established for an instantaneous solution to be accurate. Initializing LES simulations can be difficult for the same reason inlet conditions are not practical. Either an existing 
spatially correct solution must be used as an initial flow field, or the flow will need to develop for a long enough time to become statistically steady. Although the instantaneous solution is constantly changing due to the nature of turbulence, statistical quantities may be constant as in fully-developed pipe flow. The time-averaged velocity profile, pressure drop, and derivatives of these values will be constant when averaged over a long enough period of time. The goal of the LES simulation is to reach this statistically steady state as quickly as possible to keep computation time to a minimum. One method for initializing the flow field in a way that allows it to develop into the statistically steady turbulent state is to use a close approximation of turbulence based on analysis of experimental flows. The core region of pipe flow may be approximated by isotropic turbulence for initialization purposes. In practice this is accomplished by superposing random fluctuations on an otherwise smooth profile.

The random fluctuation technique is used here in combination with increasing mesh refinement to cut down computation time. First, the flow is initialized using a flat velocity profile across the entire domain, but on a coarse mesh, and with random perturbations. This solution is allowed to run until statistically steady, which takes about 5000 time steps. The final state of this solution is then used as the initial condition on the final mesh. Approximately 2000 additional time steps are required for the solution to become statistically steady on the final mesh. The final state of this solution is then used as the initial condition for the simulation of interest.

\subsubsection{Solver Settings}

The LES simulation used for this research was repeated eight times, each at a different Reynolds number. All of the simulations used the same mesh and boundary 
conditions. All of the simulations also used most of the same solver settings. The settings which remained consistent through all of the simulations are shown in Table 3-2.

Table 3-2 Solver settings used for all simulations

\begin{tabular}{|c|c|}
\hline Setting & Value \\
\hline Smagorinsky Constant & 0.1 \\
\hline Advection Scheme & $2^{\text {nd }}$ Order Central Difference \\
\hline Transient Scheme & $2^{\text {nd }}$ Order Backward Euler \\
\hline Pressure Velocity Coupling & Fully Coupled \\
\hline Convergence Controls & $1 \times 10^{-5}$ RMS Momentum Residuals \\
\hline Average Courant Number & 0.6 \\
\hline
\end{tabular}

The correct Smagorinsky constant depends on the type of flow being modeled.

Other researchers have shown that the best results for pipe flow are obtained when using a value of 0.1 [22]. The solution is also not particularly sensitive to this parameter. The value of 0.1 is the default in CFX and is the value used here.

The advection scheme deals with the discretization of the governing transport equations. Most CFD models will use upwind schemes when advection dominates over diffusion and the grid is aligned with the flow. In LES models, however, there is localized swirling and velocity components in directions other than that of the bulk flow. The central difference scheme is recommended for use in LES models in the best practices guide included with CFX [19]. This avoids the numerical dissipation of the upwind schemes from flow not aligned with the grid. The $2^{\text {nd }}$ order central difference scheme is therefore selected when setting up these simulations. 
The transient scheme determines how the portions of the governing equations dealing with derivatives in time are discretized. Because the flow field at any point in time depends only on the past flow field, and not future events, it is possible to use explicit techniques that require no iteration and are therefore computationally efficient. The explicit techniques are only conditionally stable. This becomes particularly problematic when using fine spatial discretization which might require unreasonably small time steps to maintain stability. Fully implicit first order methods are too diffusive for use with LES and will unnaturally damp out the turbulence. The higher order implicit methods work best for LES.

CFX uses a fully coupled approach for pressure and velocity. Segregated solvers employ a solution strategy where the momentum equations are first solved, using a guess for the pressure field, and an equation for a pressure correction is obtained. The pressure field is then updated to the corrected value and the procedure is repeated. Because of the 'guess-and-correct' nature of the linear system, a large number of iterations are typically required in addition to the need for selecting appropriate relaxation parameters for the variables. CFX uses a coupled solver, which solves the set of discretized equations as a single system. This solution approach uses a fully implicit discretization of the equations at any given time step. This reduces the number of iterations required to calculate the solution for each time step in a transient analysis.

Because of the iterative solution procedure required by the implicit discretized equations, the solutions approach a final value as the procedure progresses. During the first iterations there is an imbalance in the equations that is indicated by the residuals. Though additional iterations, the value of the residuals decreases as the solution 
approaches completeness. The solver determines whether the solution at a particular time step is complete by checking to see if the residuals are below some threshold value. The default threshold value is $1 \times 10^{-4}$, and is generally sufficient, but a more conservative value of $1 \times 10^{-5}$ was used. Keeping the time steps smaller will allow faster convergence and a looser convergence criteria can be used.

The Courant number is a dimensionless parameter that indicates the relative size of the time step for the given mesh and flow conditions. It is defined as:

$$
C=U \frac{\Delta t}{\Delta x}
$$

where $U$ is the fluid velocity, $\Delta t$ is the time step size, and $\Delta x$ is the grid size in the flow direction. This number indicates how many grid lengths a fluid element travels each time step. Smaller Courant numbers are required for stability in explicit transient schemes. In LES models the Courant number should be kept small enough to require only three to five iterations, or coefficient loops, per time step [19], and a value between $0.5-1.0$ is generally adequate. Using a Courant number of $0.6-0.7$ for the simulations used in this research allowed the solution to be converged at each time step after at most five iterations per step.

In addition to the consistent solver settings, the fluid properties were kept constant for each simulation. The working fluid was water with density of $997 \mathrm{~kg} / \mathrm{m}^{3}$, and dynamic viscosity of $8.899 \times 10^{-4} \mathrm{~N} \cdot \mathrm{s} / \mathrm{m}^{2}$. The different Reynolds numbers were achieved by modifying the fluid velocity. The time step size was also changed to keep the Courant number the same for each simulation. The settings and Reynolds number for each simulation are shown in Table 3-3. 
Table 3-3 Settings used to vary Reynolds number

\begin{tabular}{|c|c|c|c|}
\hline Reynolds Number & Mass Flow Rate & Average Velocity & Time Step \\
\hline 91,000 & $6.454 \mathrm{~kg} / \mathrm{s}$ & $0.8 \mathrm{~m} / \mathrm{s}$ & $1.25 \times 10^{-3} \mathrm{~s}$ \\
\hline 114,000 & $8.068 \mathrm{~kg} / \mathrm{s}$ & $1.0 \mathrm{~m} / \mathrm{s}$ & $1.0 \times 10^{-3} \mathrm{~s}$ \\
\hline 227,000 & $16.14 \mathrm{~kg} / \mathrm{s}$ & $2.0 \mathrm{~m} / \mathrm{s}$ & $5.0 \times 10^{-4} \mathrm{~s}$ \\
\hline 341,000 & $24.20 \mathrm{~kg} / \mathrm{s}$ & $3.0 \mathrm{~m} / \mathrm{s}$ & $3.33 \times 10^{-4} \mathrm{~s}$ \\
\hline 455,000 & $32.27 \mathrm{~kg} / \mathrm{s}$ & $4.0 \mathrm{~m} / \mathrm{s}$ & $2.5 \times 10^{-4} \mathrm{~s}$ \\
\hline 569,000 & $40.34 \mathrm{~kg} / \mathrm{s}$ & $5.0 \mathrm{~m} / \mathrm{s}$ & $2.0 \times 10^{-4} \mathrm{~s}$ \\
\hline 796,000 & $56.48 \mathrm{~kg} / \mathrm{s}$ & $7.0 \mathrm{~m} / \mathrm{s}$ & $1.43 \times 10^{-4} \mathrm{~s}$ \\
\hline $1,140,000$ & $80.68 \mathrm{~kg} / \mathrm{s}$ & $10.0 \mathrm{~m} / \mathrm{s}$ & $1.0 \times 10^{-4} \mathrm{~s}$ \\
\hline
\end{tabular}

\subsection{General Observations}

In order to ensure that the LES model is working appropriately, it is useful to examine the general characteristics of the flow. Eddies ranging in size from the pipe diameter to the grid spacing should be resolved if the model is working correctly. A contour plot of the fluid velocity as seen in cross sections through the fluid domain helps to visualize the flow and confirms the presence of the expected eddies. Such a plot is shown in Figure 3-2 for the initial and final time steps ( $0.0 \mathrm{~s}$ and $0.25 \mathrm{~s}$ ) of the $4 \mathrm{~m} / \mathrm{s}$ solution. Both end views and side views are shown at each time step. The large eddies are clearly visible as indicated by the spatial variation in the fluid velocity.

\subsection{Model Verification}

In CFD simulations, or any numerical model, there are differences between the exact analytical solution of the modeled differential equations and the fully converged 

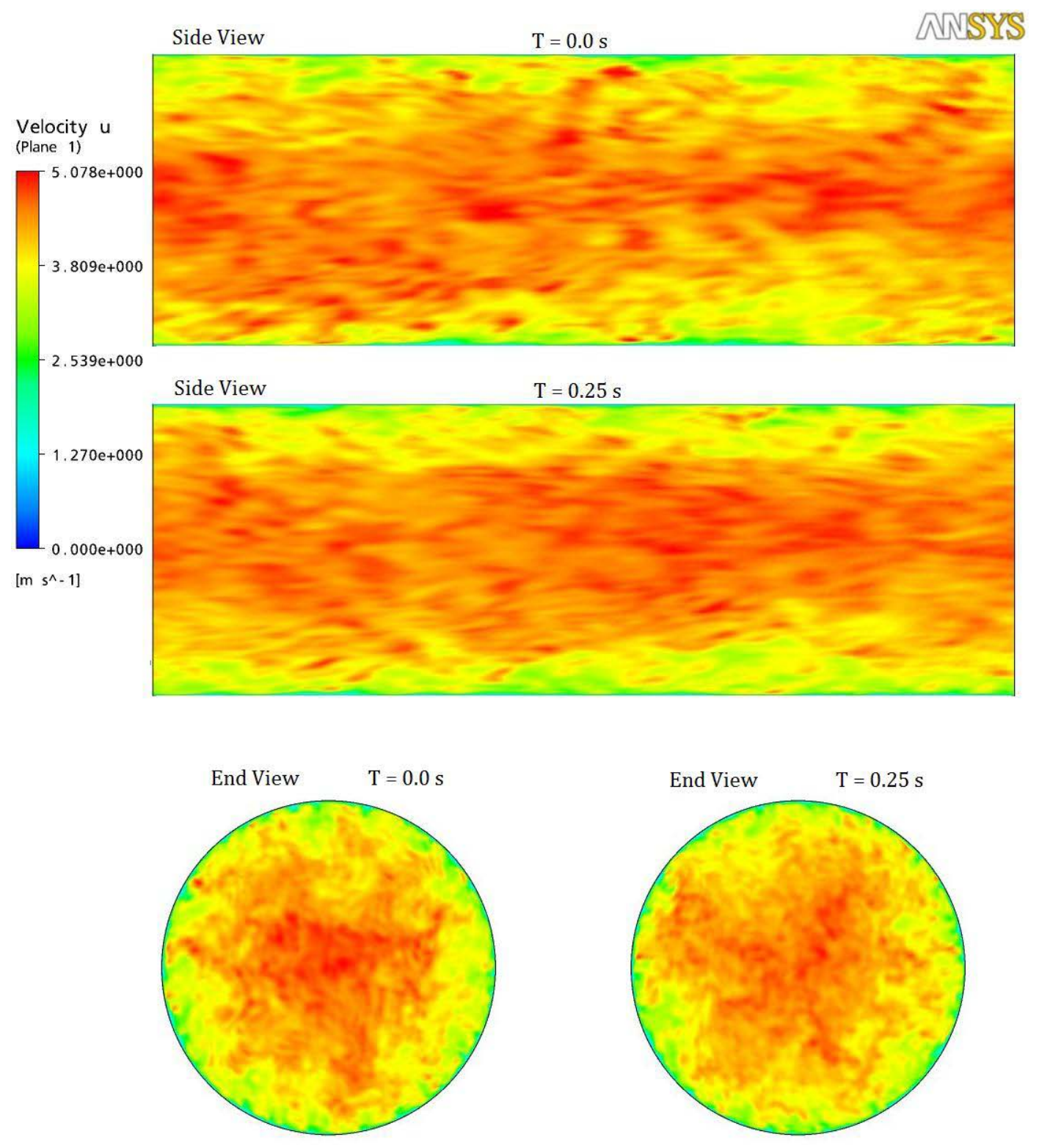

\section{Figure 3-2 Contours of fluid velocity at first and last time step of $4 \mathrm{~m} / \mathrm{s}$ solution}

solution of their discrete representations. These differences are referred to as discretization errors. These errors in the values of the principle variables being solved for are both generated by localized sources and propagated throughout the solution domain. Localized sources of error result from the higher-order terms that are excluded from the modeled equations when they are discretized. Error propagation results from the form of 
the terms that are included in the discrete approximations. Both error sources and propagation are affected by the solution and mesh distributions. The model can only be successful if it arrives at a solution that is suitably close to the true solution to the original equations. Because the discretization errors are related to the mesh size, time step size, and solution procedure, the accuracy of the solution can be estimated by analyzing how the numerical solution changes in response to changes in grid density, time step, and convergence criteria. This process is called verification, and it allows one to express confidence that the numerical model gives a correct solution to the governing system of equations.

\subsubsection{Grid Size}

The previously described mesh that was used for all of the simulations contains approximately $1.9 \times 10^{6}$ nodes. Two additional meshes were produced with the same general O-grid structure, but containing fewer nodes, and therefore larger mesh spacing in all dimensions. The other meshes contain approximately $6 \times 10^{4}$ and $4 \times 10^{5}$ nodes respectively. An image comparing the three meshes is shown in Figure 3-3.
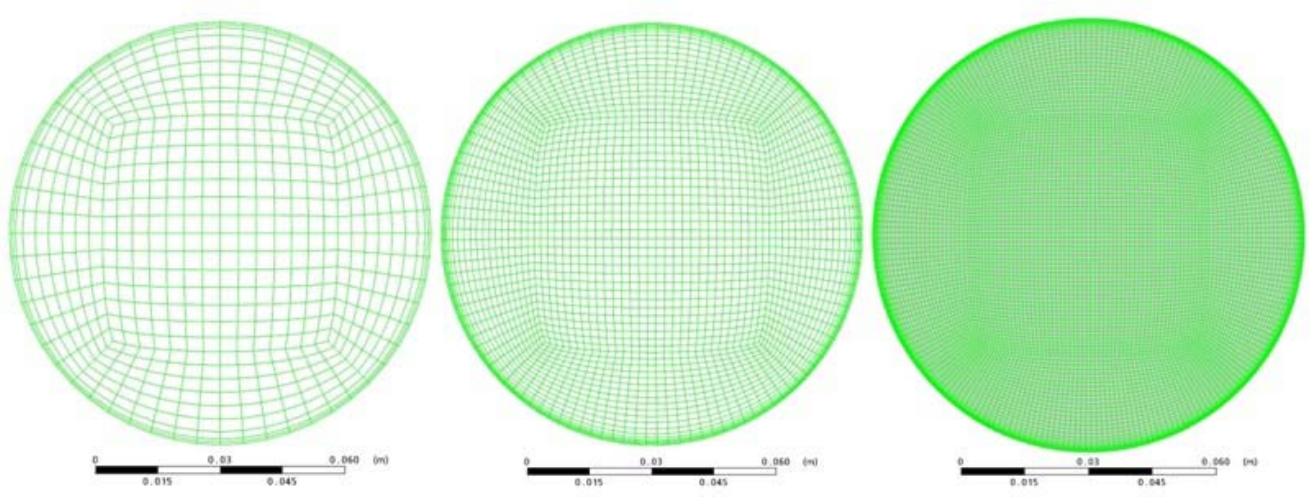

Figure 3-3 Meshes used for verification 
The three meshes were each used for a complete simulation including the initialization procedure mentioned above. The course mesh (6x10 $0^{4}$ nodes) was used to initialize all of the comparative simulations for 5000 time steps. The medium mesh ( $4 \times 10^{5}$ nodes) and final mesh $\left(1.9 \times 10^{6}\right.$ nodes) were each run for 2000 additional time steps to achieve statistically steady flow variables. Finally, all meshes were used in simulations lasting 1000 time steps during which mean flow parameters were calculated. These mean flow variables are compared for verification purposes.

The first comparison is of the time-averaged velocity profiles from the $0.8 \mathrm{~m} / \mathrm{s}$ solution as shown in Figure 3-5. The velocity profiles all appear very similar, especially those found on the medium and fine meshes. However, there are some slight differences. Both the medium and coarse meshes tend to modestly under-predict the velocity gradient near the wall. This is to be expected since the wall region is not as well refined. As a result of the inadequate wall resolution, the solver applies wall treatment using an assumed log-law profile. This causes changes in the rest of the velocity profile as well. The coarse mesh compensates with a steeper log region and has a similar core, or wake, as the fine mesh. The medium mesh keeps the same wall treatment as the fine mesh resulting in a similar log region, but over-predicts velocity in the wake. Overall, the velocity profile does not seem to be particularly sensitive to mesh refinement at these levels. The maximum velocity difference between the coarse mesh and fine mesh is about $4 \%$, and the maximum difference between the medium mesh and fine mesh is $2 \%$.

Another flow parameter that can be compared for the three meshes is the average wall shear stress. This value is averaged in time, and over the entire wall surface. Table 3-4 shows the difference in wall shear stress for the different grids. The wall shear stress 


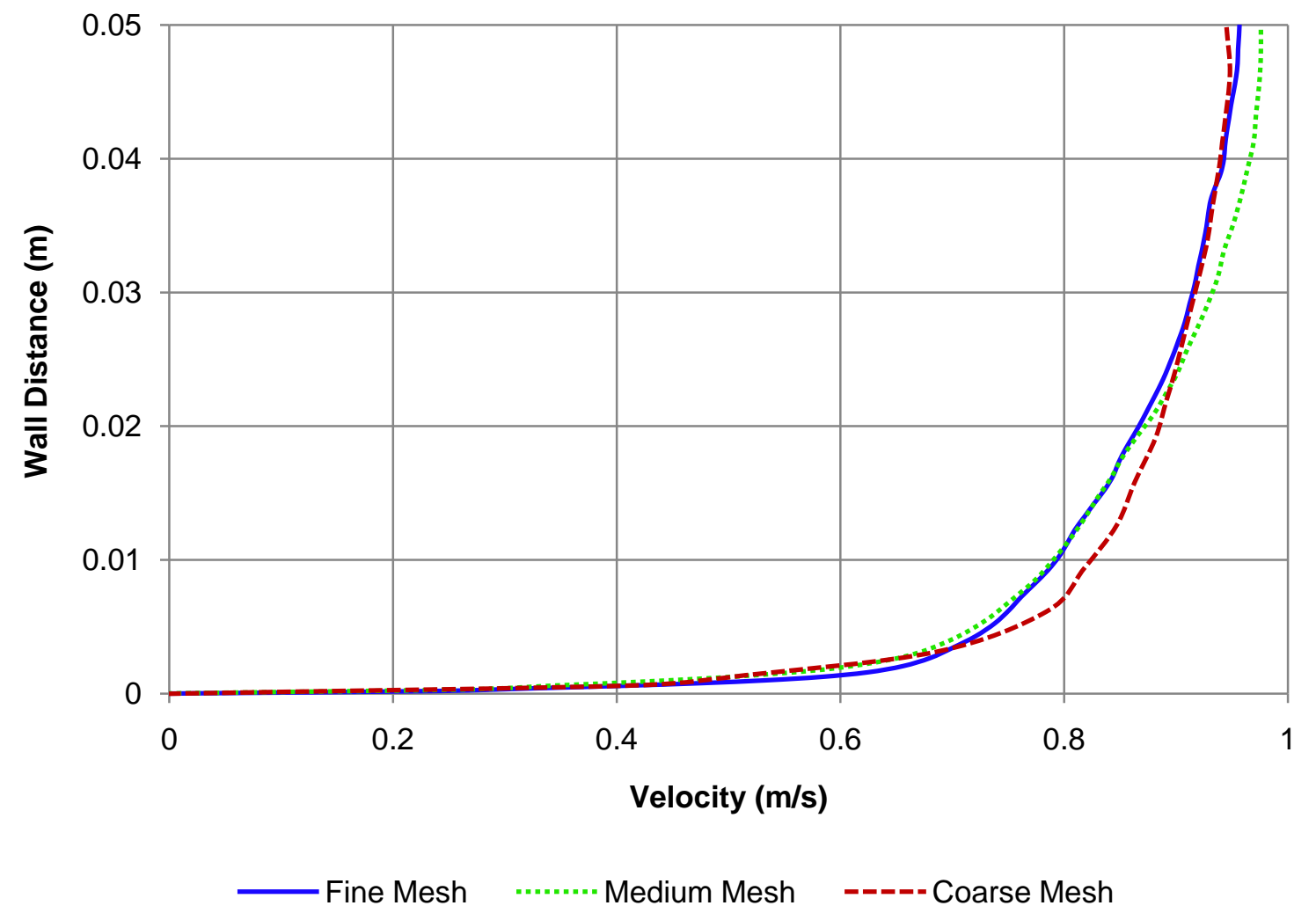

Figure 3-4 Grid resolution comparison: Time-average velocity profile from 0.8 $\mathrm{m} / \mathrm{s}$ solution

difference between the medium mesh and fine mesh are nearly negligible due to the operation of the wall function. Although it may appear the coarse grid is sufficient, the fine grid is used to keep the lower near wall $r^{+}$value at the highest Reynolds number.

Table 3-4 Wall shear stress for different meshes

\begin{tabular}{|c|c|c|}
\hline Number of Grid Nodes & Wall Shear Stress & \% Difference \\
\hline 60,000 & $1.66 \mathrm{~Pa}$ & $+3.75 \%$ \\
\hline 400,000 & $1.59 \mathrm{~Pa}$ & $-0.63 \%$ \\
\hline $1,900,000$ & $1.60 \mathrm{~Pa}$ & - \\
\hline
\end{tabular}


Because the pressure fluctuations at the wall are the driving force for pipe vibration, it is useful to consider how sensitive the amplitude of the pressure fluctuations is to the grid size. The averaged standard deviation of the wall pressure fluctuations, $P^{\prime}$, is shown in Table 3-5 for the three meshes.

Table 3-5 Pressure fluctuation amplitude for different meshes

\begin{tabular}{|c|c|c|}
\hline Number of Grid Nodes & $P^{\prime}$ & \% Difference \\
\hline 60,000 & $4.49 \mathrm{~Pa}$ & $-0.44 \%$ \\
\hline 400,000 & $4.54 \mathrm{~Pa}$ & $+0.67 \%$ \\
\hline $1,900,000$ & $4.51 \mathrm{~Pa}$ & - \\
\hline
\end{tabular}

\subsubsection{Time Step}

The numerical solution accuracy depends not only on the grid resolution, but the time step resolution as well. The size of the time step is important for convergence of the iterative solution techniques, and in transient simulations it is important to the values of the principle variables. LES in particular suffers from too much diffusion if the time step is too large, or if lower order transient discretization schemes are used. The effect of the time step is determined by comparing velocity profiles as was done when comparing grid resolution effects. In this case, three different time step sizes were evaluated: $0.005 \mathrm{~s}$, $0.0015 \mathrm{~s}$, and $.0005 \mathrm{~s}$. These time steps correspond to Courant numbers of 1.87, 0.56, and 0.19 respectively. There is no noticeable difference in the velocity profile for any of these time steps, as illustrated in Figure 3-5. Time steps resulting in Courant numbers any higher than about 1.8 sometimes force the solver to exit because of difficulty 
converging. Solutions using a time step with Courant number any smaller than 0.1 take a very long time to reach statistically steady flow. For the simulations used in this research, the use of any stable time step does not affect the quality of the solution, but does affect how long it takes to arrive at a solution.

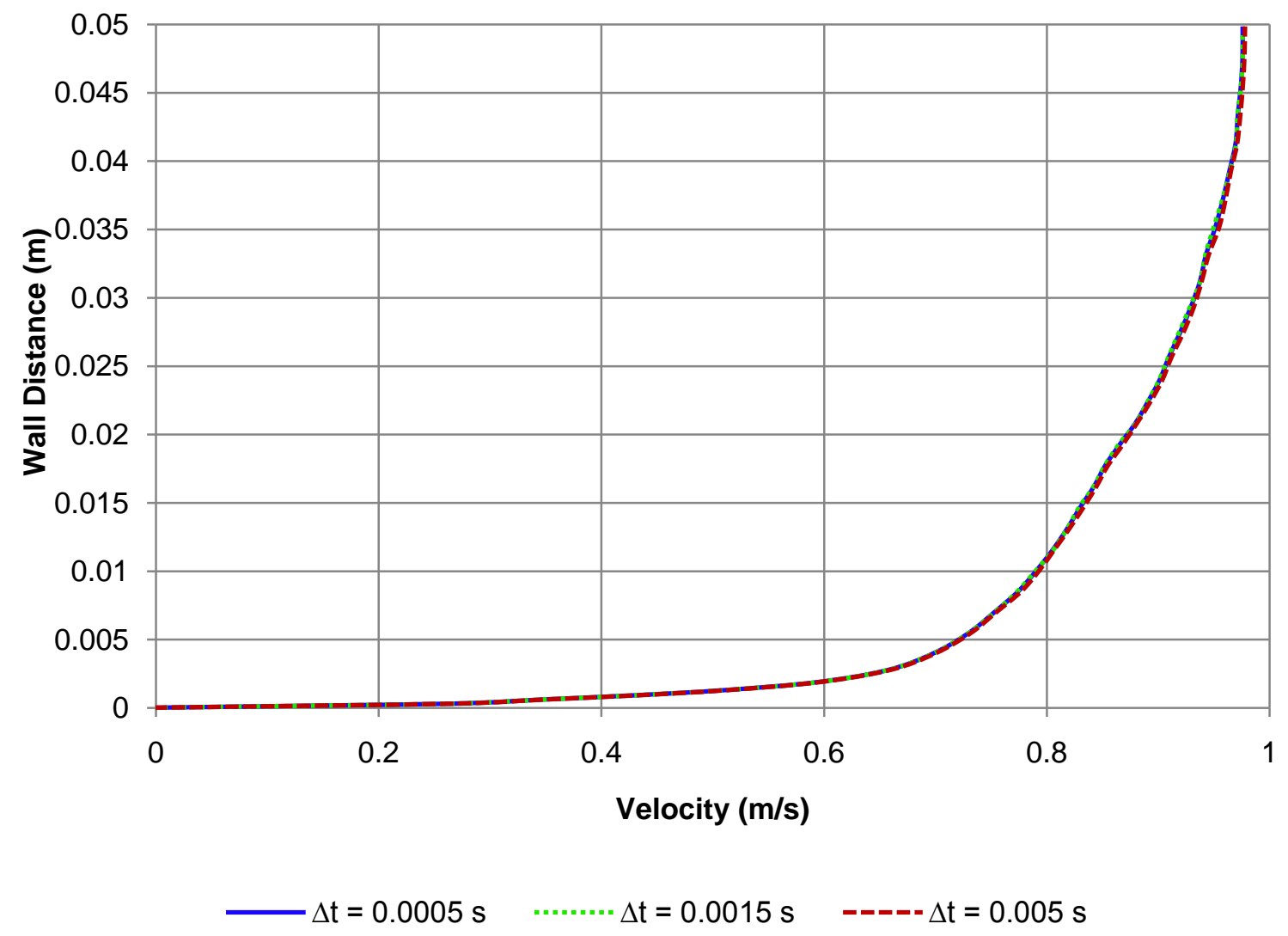

Figure 3-5 Time step size comparison: Time-average velocity profile from $0.8 \mathrm{~m} / \mathrm{s}$ solution

\subsubsection{Convergence Criteria}

The final aspect of the solution settings that needs to be verified is the iteration convergence criteria. Generally forcing the iterations to continue until a smaller residual 
value is reached before continuing to the next time step will result in solutions that are closer to the actual solution. Moving to the next time step before the solution is converged can lead to significant error propagation and erroneous results. The convergence target, set before the solution is started, controls when iterations will stop and move to the next time step. The effect of changing this target value was considered as part of the verification study. Convergence targets of $1 \times 10^{-4}, 1 \times 10^{-5}$, and $1 \times 10^{-6}$ were each used. Most time steps using the $1 \times 10^{-4}$ convergence criteria required four iterations. Requiring the residuals to reach a value below $1 \times 10^{-5}$ resulted in an average of five iterations per time step. The strictest criteria of $1 \times 10^{-6}$ required an average of six iterations per time step. A comparison of velocity profiles after several hundred time steps indicates no difference within single machine precision. Sometimes using only three or four iterations per time step resulted in a solver error causing the program to exit. Because more than five iterations did nothing to change the solution, the criterion of $1 \times 10^{-5}$ for the residuals was used in all of the simulations.

\subsection{Validation}

In addition to verifying that the numerical method has produced an accurate solution to the governing equations, it is useful to know if the equations including boundary conditions are an appropriate representation of the physical system being modeled. Because there are so many settings and techniques that can be applied so easily in a CFD simulation, the solution needs to be validated by comparison to measured results. The LES model used here has been validated using two comparisons to empirical 
data. Turbulent pipe flow has received considerable experimental attention and there is significant data available for comparison.

The logarithmic law of the wall proposed by Von Karmen [25] has been shown to fit experimental data for high Reynolds number turbulent flows. The law of the wall is expressed as a relationship between the dimensionless fluid velocity and the dimensionless wall distance. For a cylindrical pipe it is:

$$
u^{+}=\frac{1}{\kappa} \ln r^{+}+C^{+}
$$

where $C^{+}$is a constant determined experimentally to be $C^{+} \approx 5.0$, and $u^{+}$is the local time-averaged fluid velocity non-dimensionalized by the friction velocity

$$
u^{+}=\frac{u}{u_{\tau}}
$$

The law of the wall fits experimental turbulent pipe flow velocity profiles in the region above the viscous sub-layer, but does not fit the region in the center of the pipe as $r \rightarrow 0$. A better correlation that is valid for smooth pipes, including the core region, was proposed by Guo and Julien [26] and shown to fit experimental pipe data very well everywhere except near the wall. The modified log-wake law is:

$$
u^{+}=\frac{1}{\kappa} \ln r^{+}+C^{+}+2 \sin ^{2} \frac{\pi \xi}{2}-\frac{1}{\kappa} \frac{\xi^{3}}{3}
$$

where $\xi$ is the wall distance compared to the pipe radius, or:

$$
\xi=\frac{R-r}{R}
$$

If the CFD model of pipe flow is valid, it should match the experimental data, and therefore be fit closely by the log-wake law. For the LES model, the worst case simulation (where the most modeling of the small scale turbulence is done) is at the 
highest Reynolds number where the $r^{+}$value of the near wall node is highest. This simulation has the poorest grid resolution because of the higher Reynolds number and smaller turbulence scales. The velocity profile for this case with Reynolds number $R e_{D}=$ $1.14 \times 10^{6}$ is compared with the log-wake law which has been shown to yield excellent agreement with experimental data. Figure 3-6 shows this comparison. The poorer fit to the log-wake law below $r^{+} \approx 1000$ near the wall is due to the failure of the grid to fully resolve the near wall region. The LES model relies on the solver wall function which influences the velocity profile here. The rest of the velocity profile fits the log-wake law very well, indicating that the LES model is representing physical pipe flow appropriately.

The LES model was also validated by comparing the Darcy friction factor of the model with the measured friction factor for a smooth pipe. Experimental data has been obtained for a wide range of Reynolds numbers and presented on the classical Moody Diagram. The lower bound for friction factor occurs when a pipe is perfectly smooth.

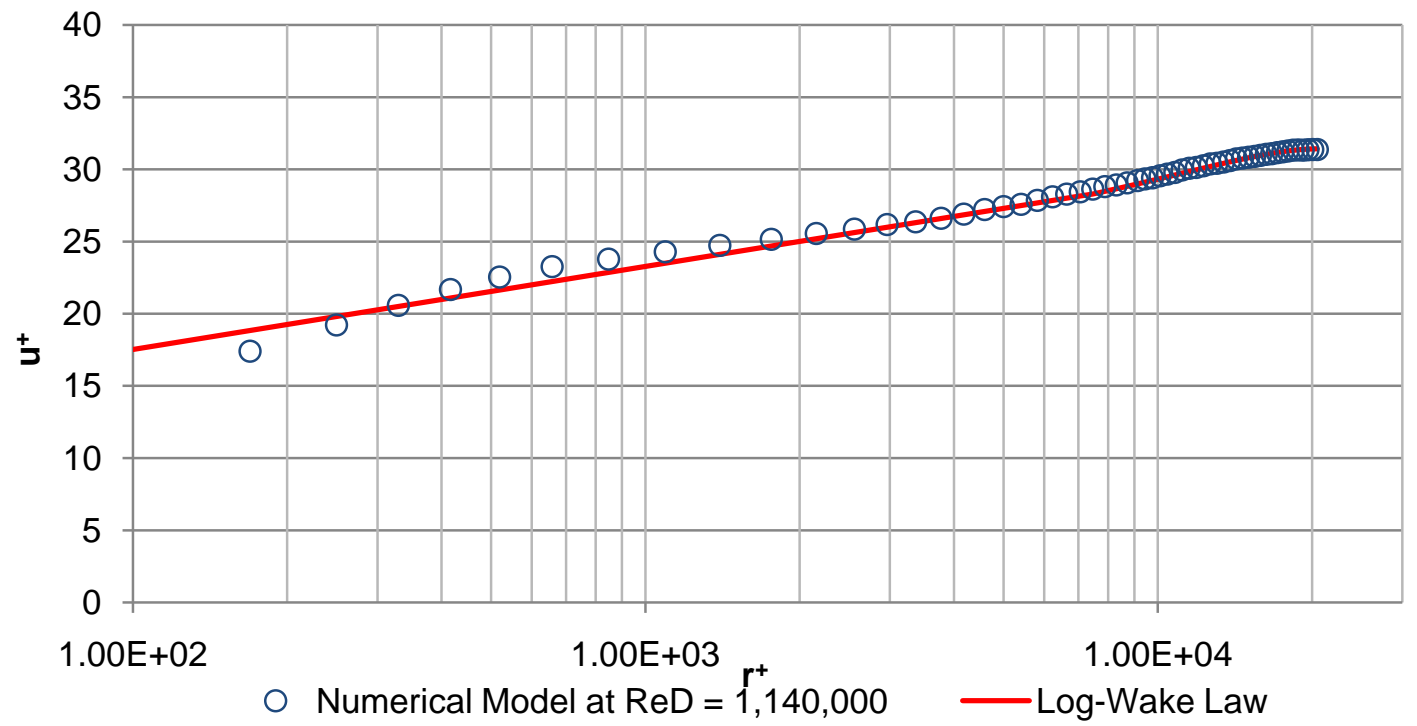

Figure 3-6 High Reynolds number velocity profile 
Because the model does not consider surface roughness effects, it will be compared to the line on the Moody diagram for perfectly smooth pipes. The friction factor in the LES simulations is determined from the wall shear stress. Tabulated values for experimental friction factor are reported in comparison to those from the model in Table 3-6. With a maximum relative error of less than $10 \%$ the numerical results are within the uncertainty range of the experimental data, again indicating an appropriate model of the physical system.

Table 3-6 Friction factor comparison with experimental data

\begin{tabular}{|c|c|c|c|}
\hline Reynolds Number & LES model & Moody Diagram & Relative Error \\
\hline 91,000 & 0.0201 & 0.0185 & $8.4 \%$ \\
\hline 114,000 & 0.0189 & 0.0179 & $5.8 \%$ \\
\hline 227,000 & 0.0163 & 0.0155 & $5.0 \%$ \\
\hline 341,000 & 0.0149 & 0.0143 & $4.1 \%$ \\
\hline 455,000 & 0.0138 & 0.0135 & $2.2 \%$ \\
\hline 569,000 & 0.0132 & 0.0130 & $1.2 \%$ \\
\hline 796,000 & 0.0123 & 0.0123 & $0.1 \%$ \\
\hline $1,140,000$ & 0.0115 & 0.0115 & $-0.2 \%$ \\
\hline
\end{tabular}




\section{FEA Structural Model}

The structural model of the pipe was created using ANSYS Multiphysics. This model experiences transient deformation in response to pressure fluctuations calculated by the fluid model. It uses the finite element method (FEM) which is also referred to as FEA, or finite element analysis, when applied to an engineering problem. This is a numerical approach which breaks the structural domain up into discrete elements and builds a system of algebraic equations relating the stress and strain of each element to that of its neighbors. Each element is required to be in balance such that the sum of all forces acting on the element is equal to the mass of the element times its acceleration. A simplistic construction of this relation is:

$$
M A=F-B V-K \delta
$$

where $M$ is the element mass, $A$ is the element acceleration, $F$ is an external applied force, $B$ is the damping coefficient, $V$ is the element velocity, $K$ is the element stiffness, and $\delta$ is the element displacement. A system of equations consisting of this relation repeated for each element in each degree of freedom must be solved, since the stiffness and displacement depend on the relative location of each element's neighbors. The solution to this system of equations returns the principle variables $A, V$, and $\delta$ for each discrete location. 


\subsection{Model Physics}

Similar to the fluid model, the structural model is solved in a defined region or domain, which is discretized into elements. Portions of the domain are defined as boundaries where the solution is restricted, or forced to match prescribed conditions. The physical properties of the material represented in the domain are specified and external loads are applied. Finally, the solver approach is chosen and the solution is calculated.

\subsubsection{Domain and Elements}

The pipe is represented in the FEA model by a two-dimensional surface. This can be thought of as a flat sheet that has been rolled up and spliced together forming a tube. The FEA domain is a cylindrical surface that corresponds to the average radial location of the pipe wall. The diameter of the FEA domain is related to the internal pipe diameter, and is simply $D+t$, where $D$ is the internal diameter and $t$ is the wall thickness. Two different classes of simulations were run that used two different domains with the difference between the two being the length of the cylindrical surface. The short pipe model is the same length as the fluid domain, being $0.3 \mathrm{~m}$ long, while the long pipe domain is $2.4 \mathrm{~m}$ long. The discretized domain has 59 circumferential segments and 45 axial segments for the short pipe. The long pipe model has 359 axial segments. This discretization results in rectangular elements that are about $5 \mathrm{~mm}$ wide and $6.7 \mathrm{~mm}$ long. A Cartesian coordinate system is used with the positive $\mathrm{x}$ direction aligned with the axis of the cylinder and the inlet being in the yz plane.

Because the FEA representation of the pipe is a two-dimensional cylindrical surface, a 2-dimensional ANSYS element is specified. The element type is a shell63 
element that has four nodes, one at each corner. Figure 4-1 shows a representation of the element with the node numbers, geometry, and local coordinate system defined. Shell63 elements have both bending and membrane capabilities and permit both in-plane and normal loads. The element has six degrees of freedom at each node: translations in the nodal $\mathrm{x}, \mathrm{y}$, and $\mathrm{z}$ directions and rotations about the nodal $\mathrm{x}, \mathrm{y}$, and $\mathrm{z}$-axes. This element can have a different thickness specified at each node, but uniform thickness was used for the simulations described here.

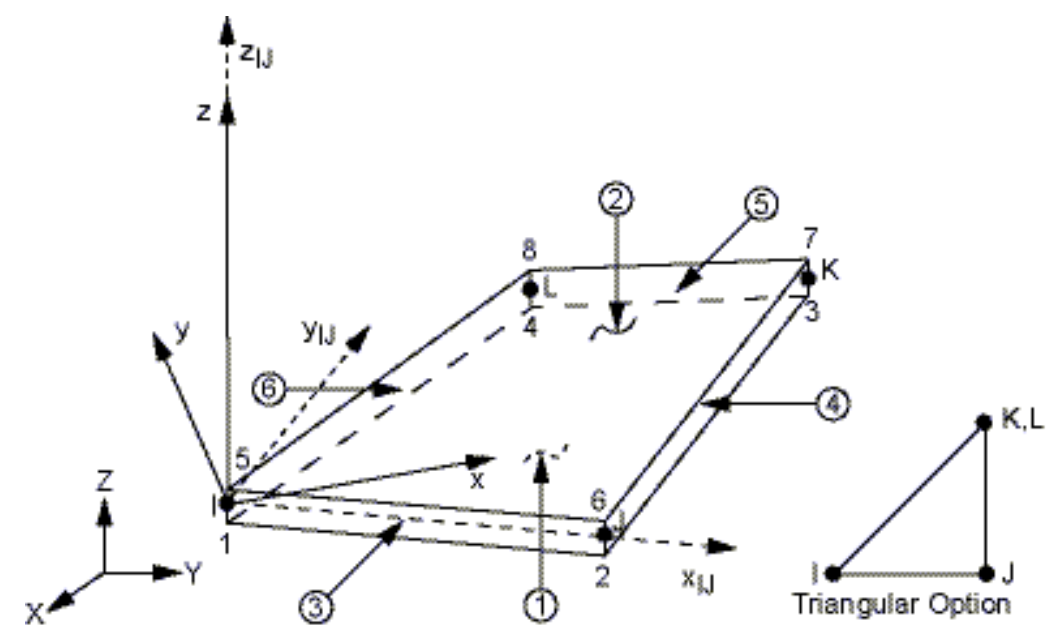

Figure 4-1 Diagram of a shell63 element

The use of shell elements reduces the computation time significantly compared to using full solid elements and a three-dimensional domain. The use of shell elements is generally considered appropriate for thin structures. In this case the pipe is being modeled as a thin-walled cylinder. As long as the pipe thickness is below about 0.1 diameters, the thin wall assumption does not significantly alter the values of quantities 
important to the solution, such as maximum hoop stress (difference of $1 \%$ for $t / D=0.1$ ) or the area moment of inertia (also different by only $1 \%$ for $t / D=0.1$ ).

A Matlab ${ }^{\circledR}$ code was written to aid in the generation of the structural mesh. This custom program was used to ensure control over the node numbering and locations, and to generate a file that could be used by the ANSYS program to import the node locations and generate the elements. A copy of the code used to create the input file is included in Appendix E.

\subsubsection{Boundary Conditions}

Boundary conditions were applied differently to the long and short pipe models. The goal of the short pipe model was to represent a very stiff pipe. This model used fixed-fixed conditions where both ends of the pipe were restricted from moving in all dimensions. Practically, this was done by limiting the degrees of freedom for all of the nodes on either end of the pipe. The long pipe model used simply supported end conditions which are intended to limit the deflection of the pipe ends, but not restrict the rotation. This model was used to represent the majority of industrial pipe systems where the pipe is supported at various locations along its length. The simply supported condition was applied by first limiting all degrees of freedom for a single node on the inlet end of the pipe. The degrees of freedom corresponding to the circumferential and radial directions ( $\mathrm{z}$ and y respectively) were limited for the corresponding node on the outlet end of the pipe. A second node on the inlet side of the pipe was also restricted in the circumferential direction to keep the entire pipe from rotating about the line between to two other restricted nodes. In total, three nodes were given displacement restrictions for the long pipe model. Figure 4-2 shows an image of the short pipe model as 
discretized, with red arrows indicating restricted degrees of freedom at the applicable nodes. The model orientation with respect to the coordinate system is also indicated by the triad in the center of the pipe inlet. Figure 4-3 shows a similar image of the long pipe model illustrating the simply supported boundary conditions. Again, the red arrows indicate restricted degrees of freedom at the nodes towards which they point.

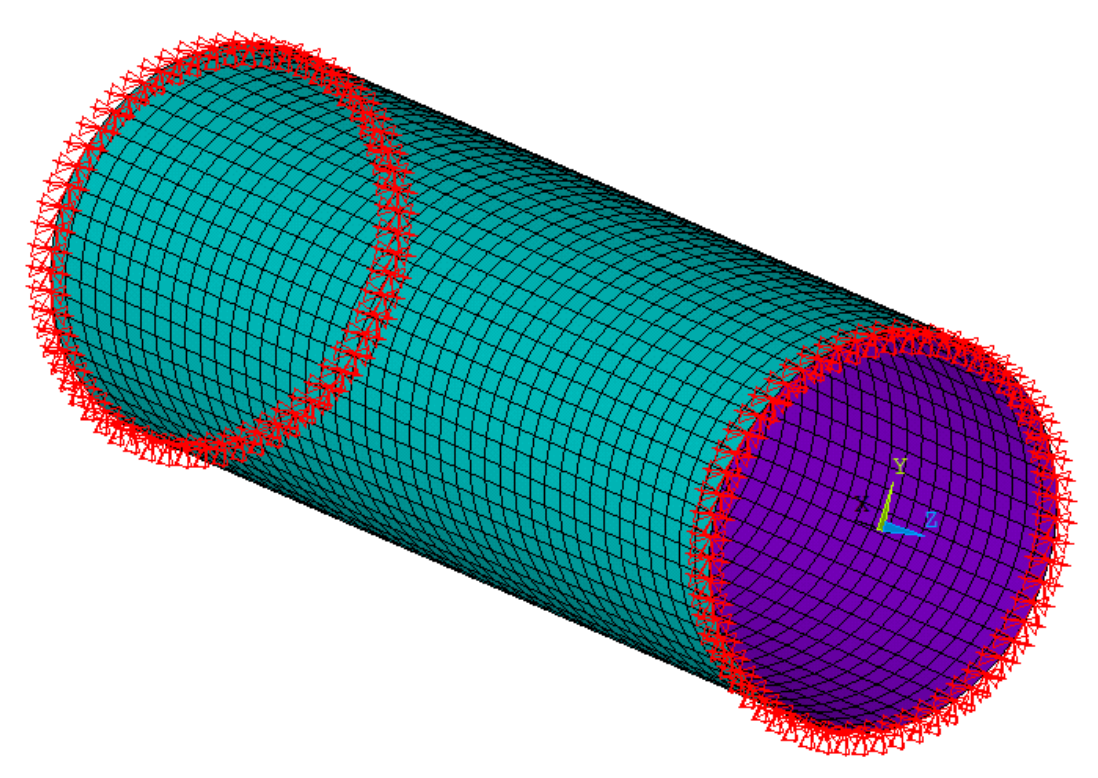

Figure 4-2 Short pipe with boundary conditions applied 


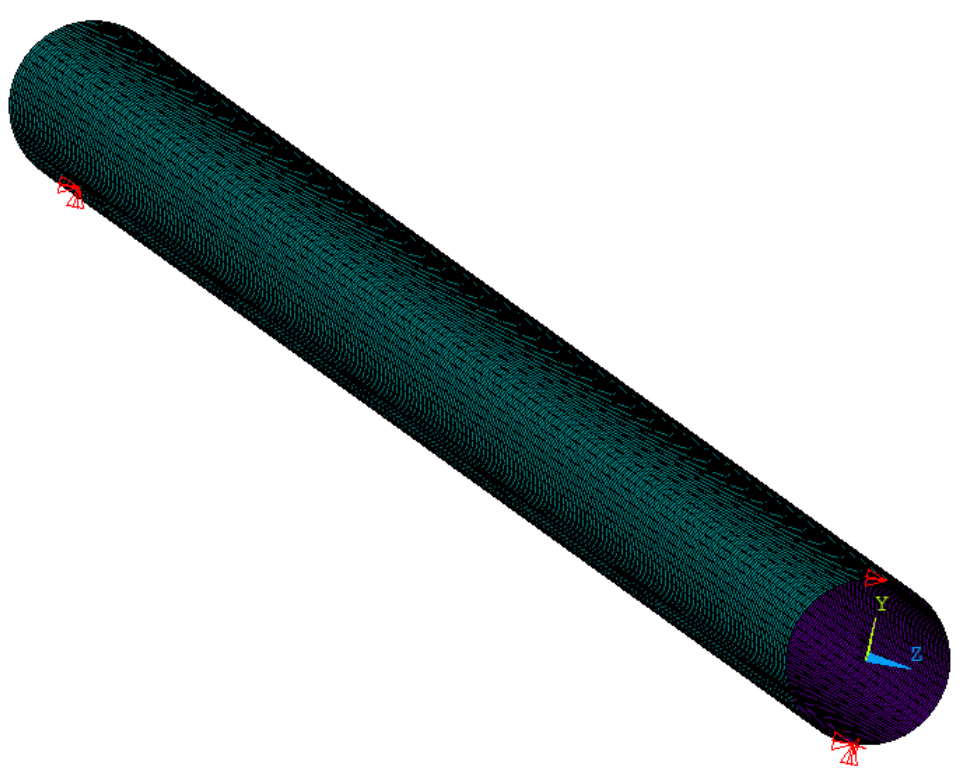

Figure 4-3 Long pipe with boundary conditions applied

\subsubsection{Solver Settings}

The ANSYS structural solver was run in batch mode to make the procedure more easily repeatable since it was run nearly 100 times to cover the range of interest for all of the variables listed in Table 1-1. A batch file was created for each run to initialize and specify all of the needed parameters including geometric and material properties. The batch file also specified the other required solver settings and input the external loads at each time step. A printout of a sample batch file is included in Appendix F.

The material properties and certain element specific constants are required before the elements are created. The only constant required for the shell63 element is the thickness. The values for the material properties and element thickness were varied from 
one simulation to the next by adjusting the parameters in the batch file. The only material properties specified for the short pipe simulations were elastic modulus and Poisson ratio. The long pipe simulations required the additional material properties of density and structural damping coefficient. The structural damping coefficient is used only in transient analysis. It is set as a constant dependant on the material composing the domain and has units of seconds. The structural damping coefficient is defined as:

$$
\beta=\frac{\zeta}{\omega_{n}}
$$

where $\zeta$ is the unitless damping ratio, and $\omega_{n}$ is the natural frequency of the pipe. This parameter controls the damping term in the system of equations, effectively providing more damping for the higher frequency modes of vibration. The material density is set to represent the equivalent density required to account for the combined mass of the pipe and the fluid. Because the cross section of the pipe is constant along its length, this parameter is constant for a given pipe material, pipe thickness, and internal fluid. The mass of the fluid per unit length contained in the pipe is:

$$
M_{f}=\rho_{f} \pi R^{2}
$$

where $\rho_{f}$ is the density of the fluid, and $R$ is the inner pipe radius. The mass of the pipe is calculated in a similar fashion. Using the thin wall assumption, the pipe mass is:

$$
M_{p}=\rho_{p} 2 \pi\left(R+\frac{t}{2}\right) t
$$

where $\rho_{p}$ is the density of the pipe material and $t$ is the pipe thickness. The equivalent density, which is assigned as a material property in the simulations, is then calculated by dividing the total mass by the cross sectional area of the pipe wall as follows: 


$$
\rho_{e q}=\frac{M_{f}+M_{p}}{2 \pi\left(R+\frac{t}{2}\right) t} .
$$

Using the equivalent density defined this way allows the mass per unit length of the empty FEA pipe to match the mass per unit length of an equivalent pipe full of fluid. For analytical models that consider the pipe as a beam, the mass per unit length is the specified parameter. Because the bending modes are expected to dominate the response of the long pipe model, the density was defined using this approach.

Some solver settings remained consistent for all of the simulations. Each simulation was set to use 1000 total time steps. The time step size was set to match that used by the fluid solution from which the externally applied loads originated. To keep the results file size manageable, the output variables were restricted to the nodal displacement, velocity and acceleration. Other solution variables such as stresses were used during calculations, but disposed of after the completion of the time step. Each of the simulations specified the use of the same method for solving the algebraic system of equations. Iterative methods generally have reduced memory requirements and work well for very large meshes. Direct methods such as the ANSYS sparse solver generally require more computer memory, but use only a single iteration for each time step. This makes these methods very fast if the computer has the available resources to allow their use. The short mesh used in these simulations consists of about 3000 elements and the long mesh has about 20,000 elements. These models have sufficiently few elements to be solved using the sparse solver, which uses Gaussian elimination to find the single solution to the system of equations.

The transient method was set differently for the long and short pipes. All of the short pipe simulations had time integration turned off, resulting in faster solution times, 
but neglecting transient effects associated with the inertia of the pipe. This was justified after discovering that inertial effects for the short, stiff pipe did not affect the solution (having the time integration setting on or off did not produce differing results). The long pipe simulations were all performed using full transient analysis by having the time integration setting turned on.

\subsection{Model Verification}

Like the LES fluid model, any FEA structural model requires verification studies to ensure the numerical method is arriving at a solution that represents a real solution to the mathematical model it approximates. This is done by determining what effects the element type, grid resolution, and time step size have on the solution. Each should then be chosen such that further refinement gives no change in the solution.

\subsubsection{Element Type}

The shell elements for these simulations use a thin-walled pipe assumption. The effects of this assumption are most noticeable for the short pipe which responds to the spatially varying pressure field differently than the long pipe. Bending deformations like those in a beam tend to dominate the long pipe motion, making local deformation less noticeable. The local deformations of the short pipe, which are more sensitive to the thin wall assumption, tend to dominate their response. An additional short pipe model using solid elements was constructed for comparison to the shell model. The solid model had three elements through the thickness of the pipe wall, with the same size elements as the shell model in the circumferential and axial directions. It was constructed such that the 
average radius matched that of the shell model. The standard deviation of the pipe wall displacement of the solid and shell models was compared at several pipe wall thickness settings. As expected, the thicker pipe wall models differed more between shell and solid meshes than thin-walled models. A plot of the relative difference in the standard deviation of the pipe wall displacement between the shell and full solid models is shown in Figure 4-4. The maximum difference occurs for the 8mm thick pipe wall, and it is less than $3 \%$. The full solid model makes fewer assumptions than the shell model and uses higher order elements so it is expected to be more accurate, but did not produce significantly different results. The difference between methods is not large enough to justify the use of the much more computationally expensive solid element model.

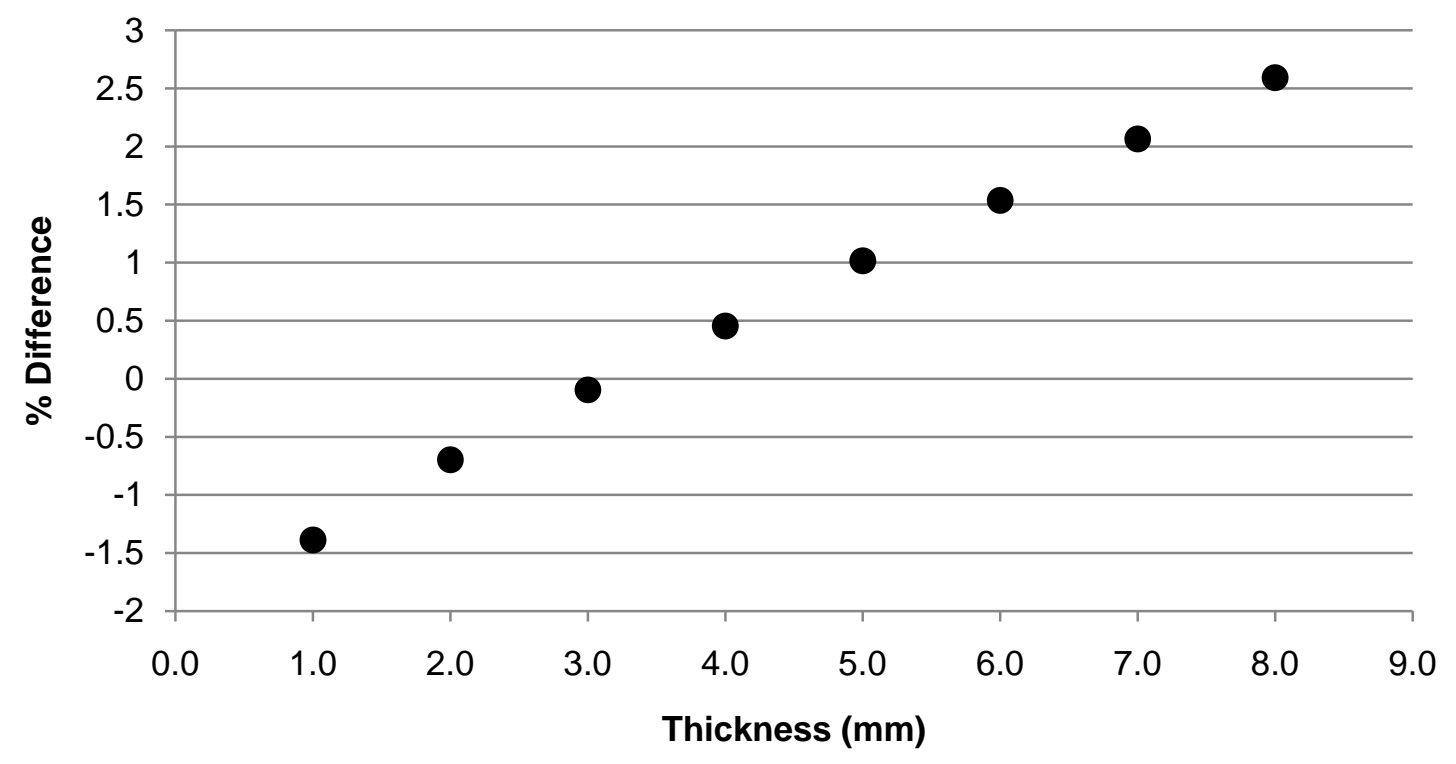

Figure 4-4 Relative difference in the standard deviation of the wall displacement between shell vs. solid element modeling as a function of wall thickness 


\subsubsection{Grid Size}

Grid independence was established by using three different grids with successive node spacing reduction by a factor of two. For the short pipe this results in a coarse grid with about 2800 nodes, a medium grid with about 11,000 nodes, and a fine grid with about 44,000 nodes. The three grids were all employed on models with pipe wall thickness of $1 \mathrm{~mm}, 3 \mathrm{~mm}$, and $6 \mathrm{~mm}$. As shown in Table 4-1, the maximum difference in the standard deviation of the pipe wall displacement between the results obtained by using the coarse mesh and those obtained by using the fine mesh is less than $5 \%$. This is considered by the author as acceptable for use in the present simulations which exhibit results varying by orders of magnitude over the ranges explored of the influencing variables. The use of the coarse mesh is justified by the relatively small difference in results compared to finer meshes, considering that it cuts the solution time by more than an order of magnitude compared to the fine mesh computation time.

Table 4-1 Structural mesh refinement effects

\begin{tabular}{|c|c|c|c|c|c|}
\hline \multirow{2}{*}{$\begin{array}{c}\text { Thickness } \\
(\mathrm{mm})\end{array}$} & \multicolumn{2}{|c|}{ Wall Displacement $\left(S^{\prime}\right)$ in $\mu \mathrm{m}$} & \multicolumn{2}{c|}{$\begin{array}{c}\text { Difference from Fine } \\
\text { Mesh Results }\end{array}$} \\
\cline { 2 - 6 } & Coarse & Medium & Fine & Coarse & Medium \\
\hline 1 & 0.193 & 0.189 & 0.184 & $4.9 \%$ & $2.7 \%$ \\
\hline 3 & 0.0214 & 0.0209 & 0.0206 & $3.9 \%$ & $1.5 \%$ \\
\hline 6 & 0.00543 & 0.00526 & 0.0052 & $4.4 \%$ & $1.2 \%$ \\
\hline
\end{tabular}




\subsubsection{Time Step}

The time step size for the transient pipe structural analysis was fixed to be the same as the time step used for the LES fluid analysis. Although it would be possible to shorten the time step for the structural FEA model, the loading conditions from the fluid solution would not be updated each time step. This would lead to unnatural behavior of the solution as the step changes in loading conditions would appear to have some very high frequency content. Although shorter time steps could not be tried effectively, the effect of the time step size was analyzed by doubling the time step and considering only every other time series data point from the fluid solution. For the pipe wall displacement there is very little noticeable effect (less than $0.2 \%$ change) from increasing the time step this way. Removing every other sample by doubling the time step has the same effect as applying a low pass filter that cuts out the higher frequency content. Because the pressure fluctuations at each node contain several samples per cycle of the highest frequency component, removing samples had very little effect. The velocity and the acceleration of the pipe wall tend to respond most readily to the high frequency content however, and these variables did change with increasing time step. The standard deviation of pipe wall velocity dropped by up to $1.7 \%$ for the worst case (pipe with highest response frequency), and the acceleration dropped by as much as $12 \%$ for the worst case. A plot of an acceleration time series for both time step sizes is shown in Figure 4-5. The loss of high frequency content for the larger time step can be seen clearly. Because of the differences that result when using a coarser time step, the original time step matching that of the fluid solution was used for all of the structural simulation to limit any potential loss in accuracy. 


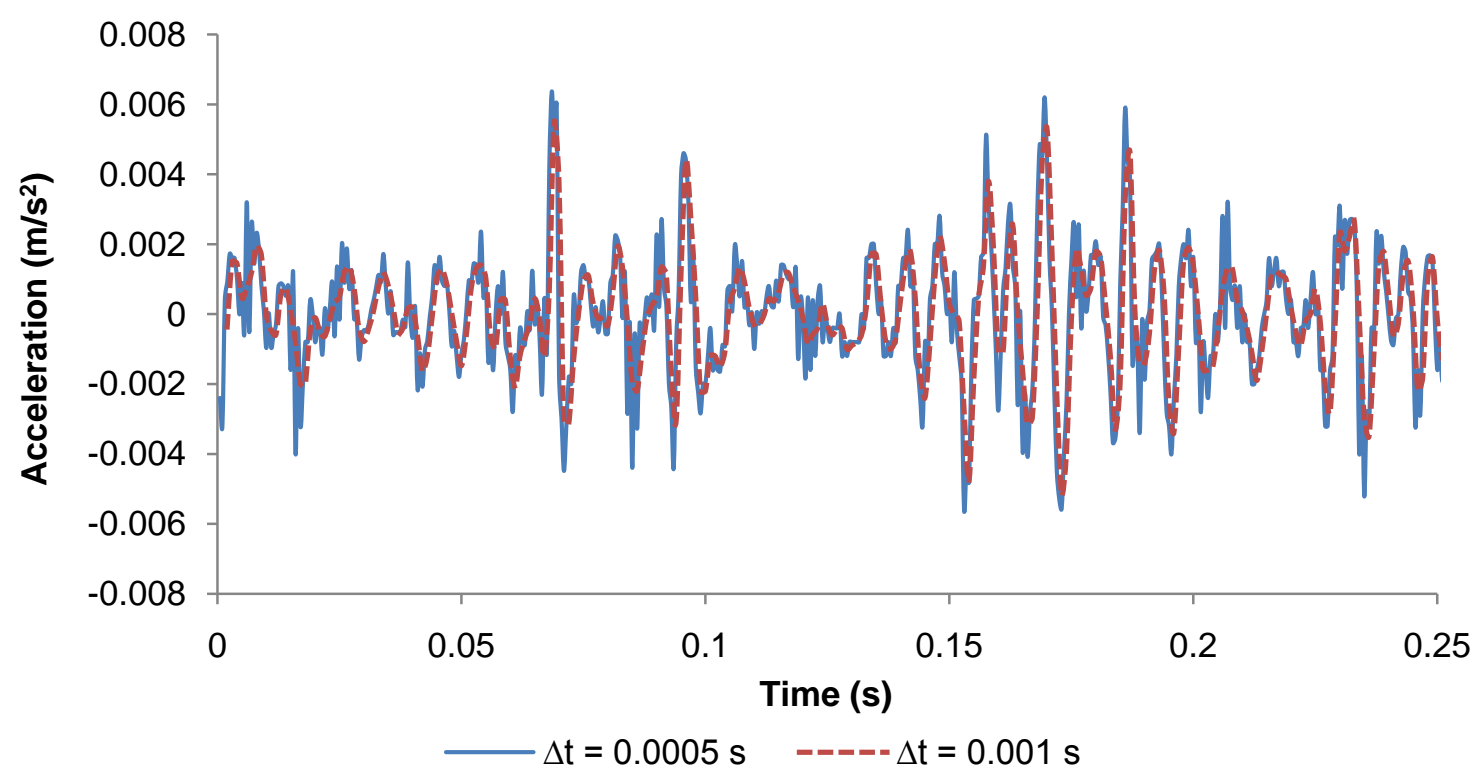

Figure 4-5 Effect of time step size on pipe wall acceleration

\subsection{Validation}

The structural model was validated by comparing results to those obtained using analytical and experimental models. The analytical validation was done by comparing the FEA results to the predicted static radial deflection due to a uniform internal pressure, and static beam deflection due to a distributed load. The experimental validation was done by comparing the frequency content of the excited transient response of a real pipe to the natural frequencies of the FEA model predicted using a modal analysis.

\subsubsection{Static Validation}

The static radial deflection of a pipe subjected to uniform internal pressure can be determined by using an analytical expression for the hoop stress and the material elastic 
modulus. Using the thin wall assumption, the hoop stress in the wall of a long cylinder is:

$$
\sigma_{\theta}=\frac{P R}{t}
$$

where $P$ is the internal pressure, $R$ is the average pipe radius, and $t$ is the pipe wall thickness. In the linear material behavior region the strain is related to the stress through the modulus, so the circumferential strain is:

$$
\delta_{\theta} \equiv \frac{\Delta C}{C}=\frac{2 \pi \Delta R}{2 \pi R}=\frac{\sigma_{\theta}}{E}
$$

where $\mathrm{C}$ is the circumference of the pipe and $\mathrm{E}$ is the elastic modulus of the pipe material. Substituting (4-6) into (4-7) and rearranging gives the incremental change in pipe radius:

$$
\Delta R=\frac{P R^{2}}{E t} .
$$

Comparing the analytical hoop stress and change in radius with the values determined by ANSYS results in a relative difference of about $2 \%$. The comparison was made for a pipe with $R=0.1015 \mathrm{~m}, t=2 \mathrm{~mm}, P=1000 \mathrm{~Pa}$, and $E=10 \mathrm{GPa}$. The summary of results from this comparison is included on Table 4-2.

The second validation study compared the bending displacement of the model with that calculated using beam theory. The maximum deflection of a simply supported beam is:

$$
\delta_{\max }=\frac{5}{384} \frac{F L^{3}}{E I}
$$

where $F$ is the total magnitude of a load distributed along the length of the beam, $L$ is the length between fixed supports, $E$ is the material elastic modulus, and $I$ is the area 
moment of inertia of the beam cross section. For a thin-walled cylindrical beam, the moment of inertia is:

$$
I=\frac{\pi}{8} D^{3} t
$$

where $D$ is the mean diameter and $t$ is the wall thickness. Finally, substituting (4-10) into (4-9) gives an analytical expression for predicting the pipe displacement due to beam-like bending:

$$
\delta_{\max }=\frac{5}{48 \pi} \frac{F L^{3}}{E D^{3} t}
$$

This value was compared for a pipe with $L=2.4 \mathrm{~m}, D=0.1015 \mathrm{~m}, t=2 \mathrm{~mm}, E=10 \mathrm{GPa}$, and a total distributed load of $F=20 \mathrm{lbs}$. The relative difference was approximately $4 \%$ as shown in Table 4-2.

Table 4-2 Structural model static validation summary

\begin{tabular}{|c|c|c|c|}
\hline Variable & ANSYS & Theory & Error \\
\hline$\sigma_{\theta}$ & $24861 \mathrm{~Pa}$ & $25375 \mathrm{~Pa}$ & $-2.0 \%$ \\
\hline$\Delta R$ & $0.1270 \mu \mathrm{m}$ & $0.1288 \mu \mathrm{m}$ & $-1.4 \%$ \\
\hline$\delta_{\max }$ & $0.457 \mathrm{~mm}$ & $0.438 \mathrm{~mm}$ & $4.3 \%$ \\
\hline
\end{tabular}

\subsubsection{Dynamic Validation}

The dynamic validation study used an impact hammer test of an experimental section of PVC pipe. The experimental pipe section had an inside diameter of $5.2 \mathrm{~cm}$ and wall thickness of $3.91 \mathrm{~mm}$. The pipe was clamped on both ends of a $1 \mathrm{~m}$ long test 
section. An accelerometer was placed at the center of the clamped section of pipe, and the pipe was struck with an impact hammer near the accelerometer. The accelerometer response was sampled at $5 \mathrm{kHz}$, and the time signal was converted to the frequency domain using a fast Fourier transform (FFT). The impact hammer procedure was repeated five times, and the five resulting spectral distributions were averaged. This is considered a small number of averages [27] and results in a noisy spectrum with poor amplitude estimates. However, the amplitude of the peaks is not of interest for this comparison, only their location.

A modal analysis was done on a structural model in ANSYS having geometry matching that of the experimental pipe. The density was set at $1300 \mathrm{~kg} / \mathrm{m}^{3}$ and the elastic modulus was set to 2.5 GPa and 3.0 GPa. Because the modulus of PVC has some variability, there is uncertainty in the modulus value used and corresponding uncertainty in the natural frequencies found by the modal analysis. The upper and lower modulus values were used to determine a range of natural frequencies that account for the uncertainty in the modulus. The natural frequencies from the modal analysis are plotted along with the spectral distribution from the experimental accelerometer measurement in Figure 4-6. The natural frequencies from the modal analysis of the ANSYS model fall fairly close to the peaks in the experimental data, indicating the experimental system has natural frequencies similar to the numerical model. Some of the apparent peaks from the experimental data may be noise due to the insufficient number of averages.

Discrepancies could also be caused by differences in the experimental boundary conditions which may allow additional modes of vibration that are restricted in the FEA model. 


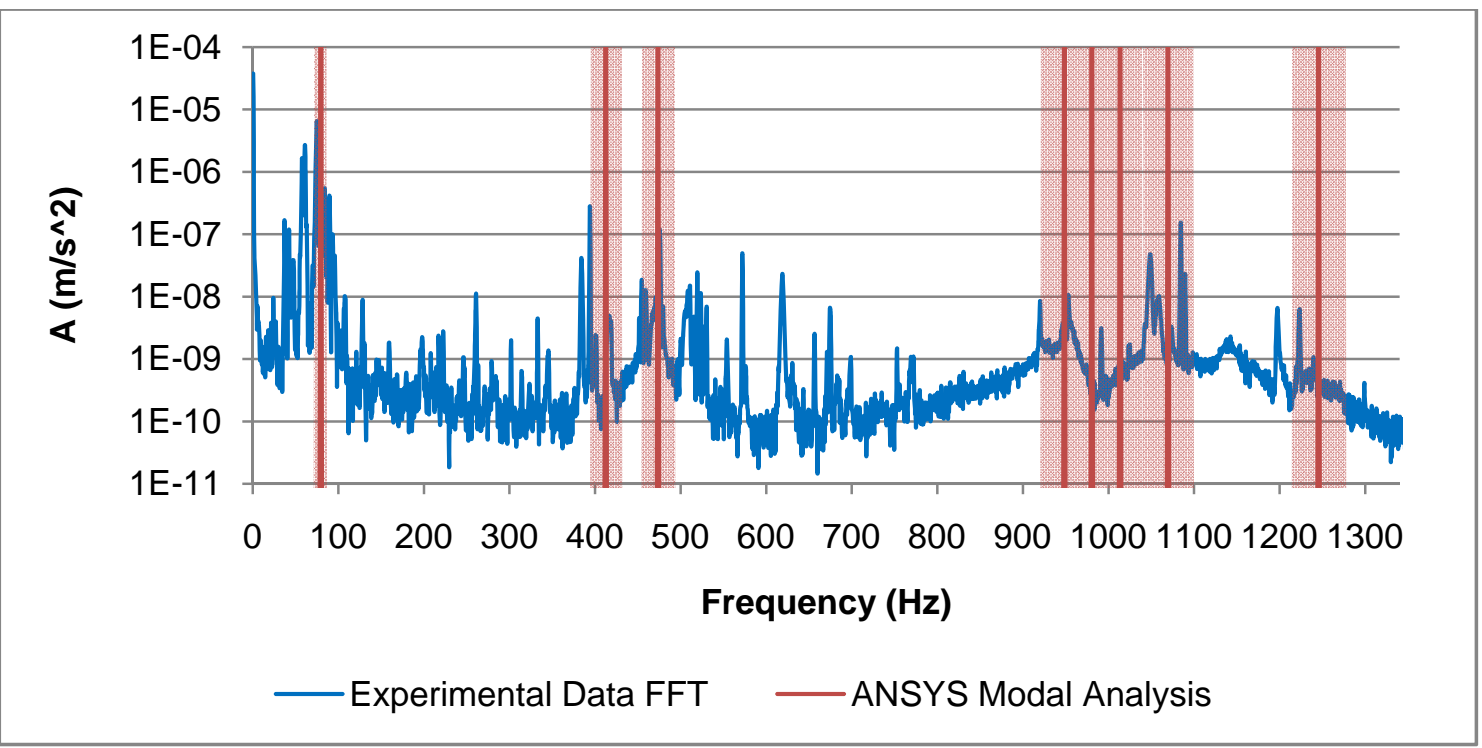

Figure 4-6 Comparison of ANSYS and experimental natural frequencies (lighter bands indicate uncertainty range in ANSYS predictions) 


\section{FSI Coupling Procedure}

The LES fluid model and FEA structural model are linked through two different coupling approaches. The first approach passes the pressure at the pipe wall from the fluid solution to the structural model as a set of external loads. The structure is then allowed to respond to the loads that are updated at each time step. Because the structure responds to the fluid solution, but the fluid solution is not influenced by the structural motion, this is called a one-way coupling approach. The second approach includes the additional step of passing the structural motion of the pipe wall back to the fluid model by deforming the fluid mesh at the wall boundary. This two-way approach allows both the structural and fluid models to respond to each other and is sometimes referred to as a fully coupled FSI model. The application of both approaches is explained in detail in this chapter.

\subsection{One-Way Coupling}

The one-way approach is simpler in theory and makes more assumptions than the two-way approach. It assumes that the structural deformations are small enough that they do not influence the flow field. It has been suggested that pipe wall deflections smaller than the viscous sub-layer thickness will not influence the turbulence generation of the flow $[9,11]$. Although the one-way model is simpler, application of the technique 
actually requires more effort than the two-way approach in order to take advantage of the potential computational benefits it offers. The one-way approach was used for nearly all of the simulations because of the significant reduction in computation time. The fluid model takes approximately 40 times longer to solve than the structural model. Because the one-way approach requires the fluid model to be solved only once, it is a much more efficient way to explore pipe geometric and material parameters.

\subsubsection{Pressure Extraction}

CFD models using a periodic boundary condition on the inlet and outlet suffer from a potential for "floating" pressure in the domain. A valid pressure field that satisfies the governing equations can have any constant added to it and still satisfy the equations unless a boundary condition involving pressure is included. Most CFD models include a pressure boundary somewhere to fix this constant. The LES model with a periodic inlet/outlet fixes the pressure at a single node to always be a certain value. The entire pressure field is then determined relative to this reference pressure. Unfortunately, the structural model requires the loads to be defined relative to a fixed external pressure. This requires the pressure field from the fluid solution to be transformed in terms of a new reference before use in the FEA model. The transformation is made by adding the absolute pressure at the fixed node to the entire pressure field at each time step. The absolute pressure at the fixed node is taken to be the negative of the volume averaged pressure so the new pressure field needed by the structural model can be calculated as:

$$
P_{\text {new }}=P-\bar{P}
$$

where $P$ is the pressure value stored in the fluid solution and $\bar{P}$ is the instantaneous pressure averaged over the entire fluid domain. The calculation of $\bar{P}$ must be done 
separately for each time step when making the transformation. The CFX post processor includes a custom function option that allows this new variable to be defined and exported for all nodes on the wall. The transformed pressure data for use in the structural model was exported to a series of text files, each containing the pressure data for a particular time step. Because the pressure extraction procedure from CFX needed to be repeated 1000 times for each of eight flow solutions, a script was written that would automate the procedure. Doing this through the normal menus in the post processer would not have been practical. A copy of the script is included in Appendix C.

\subsubsection{Pressure Mapping}

The long pipe structural model is eight times as long as the fluid model. This requires the pressure field to be repeated eight times in the axial direction. There are also many fewer surface nodes in the structural model than in the fluid model. The fluid model contains four nodes for every structural node in both the circumferential and axial directions. The fluid model requires the higher grid resolution to get the proper turbulent structures to develop and to produce the proper pressure field. Physically, the pipe wall acts to smooth out or average the spatially varying pressure fluctuations. This is one reason why the FEA model can use a coarser mesh and still accurately represent the physical system.

For all of the one-way simulations, the transformed pressure data from each solution was imported into Matlab ${ }^{\circledR}$ and stored as a 236x180x1000 array corresponding to the 236 circumferential nodes, 180 axial nodes, and 1000 time steps from the fluid solution. The pressure data was then reduced using an area averaging scheme. Overlapping $5 \times 5$ sections of the array were averaged to produce a single pressure value 
for that region to be mapped to a structural node. This resulted in a 59x45×1000 array of pressure data corresponding to the coarser structural mesh. An example of the original fluid pressure field and the reduced field for the structural mesh is shown in Figure 5-1 for comparison. The reduced pressure field was then repeated axially eight times to match the longer structural domain. The reduced pressure field was finally converted to forces by multiplying by the structural element area at each node and decomposed into y and $\mathrm{z}$ components for easier application in ANSYS. A file including a series of commands to apply all of the nodal forces was created for each time step. These files were then imported for use by ANSYS at each time step during the solution of the structural model to apply external loads. The pressure mapping procedure required the use of a Matlab program to import the pressure data, manipulate it to fit the structural mesh, and write the ANSYS input files. A copy of the code is included in Appendix D.
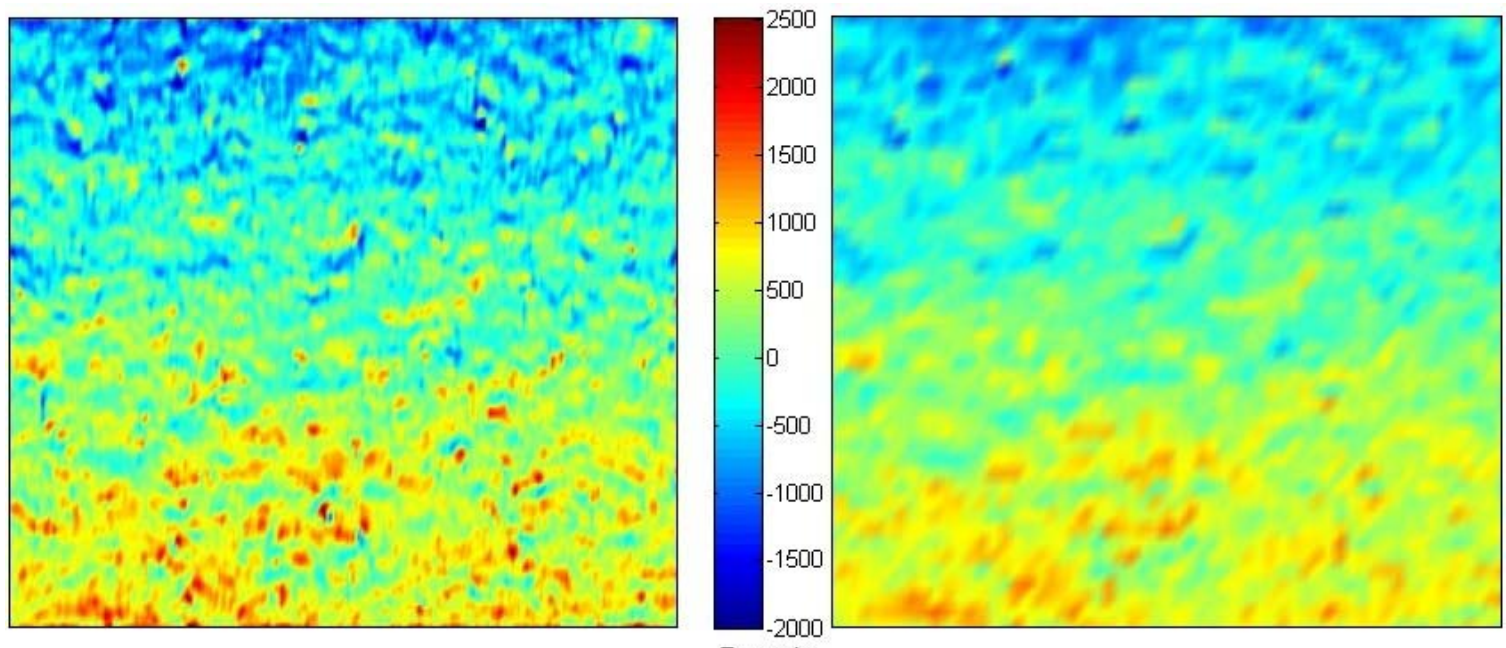

Pascals

Figure 5-1 Pressure field before and after averaging (original field on left) 


\subsection{Two-Way Coupling}

The two-way procedure is considered necessary when the structural deformation will affect the flow field. Even if the maximum pipe wall displacement is smaller than the viscous sub-layer, the pipe wall motion causes a non-zero radial velocity at the wall. The no-slip condition forces this velocity to be matched by the fluid adjacent to the wall. Also, because the LES model calculates the instantaneous velocity and pressure values (it is a time accurate transient technique), even small changes in the wall boundary location may have an effect on the flow field. For these reasons, the two-way solution may not produce the same results as the one-way procedure even with very small pipe wall displacement. The two-way procedure was used as a way to validate the one-way FSI model by determining the magnitude of the difference in results. If using the two-way procedure does not produce different results, it is not practical because of the significantly higher computation cost over the one-way model.

\subsubsection{Application}

The two-way technique does not require the pressure extraction and mapping procedures because ANSYS has a built-in function that takes care of interpolating between dissimilar meshes between time steps. The built in interpolator is slow compared to the Matlab program used in the one-way procedure, but because it can be done without exiting the fluid solver it makes the two-way procedure a practical possibility. The fluid solver requires a few additional settings to let it know which boundaries will deform in response to the structural solution. It also needs to be told to pass the pressure data each time step through the interpolator to the structural solver. The 
structural model also requires additional settings so it can pass the wall displacement through the interpolator to the fluid mesh. It must be set to accept external loads each time step from the fluid solver rather than from existing files.

The two-way model was used for only 600 time steps because the results files it generated became too large to open for post processing and extracting pipe wall motion data. It required about two weeks of continuously running on four processors, which is almost six times as long as a normal fluid solution alone. The additional time was a result of the iterative process required to have the boundary values of both the fluid solver and structural solver agree. The multiple iterations cause the fluid model to be solved about five times for each time step. The results file for 600 time steps was over 140 Gigabytes.

\subsubsection{Comparison With One-Way Approach}

The two types of coupling procedures were compared by first allowing the flow field to become statistically steady with no coupling settings. This flow field was then used as the initial condition for a flow solution to be used in a one-way approach, and for the initial state of the fully coupled two-way flow solution. The two-way procedure was run to completion as described. The one-way procedure was then applied to extract and map the pressure data from the uncoupled flow solution. The simulations were both done using the short pipe mesh so transient structural effects were not considered in the oneway model. The average fluid velocity was set to $2 \mathrm{~m} / \mathrm{s}$ and the fluid properties were those of water as specified in chapter 3. The elastic modulus of the pipe was set to 3.7 GPa and the wall thickness was set to $6 \mathrm{~mm}$. The pipe material density for the two-way model was set to $1300 \mathrm{~kg} / \mathrm{m}^{3}$. 
The pipe wall motion was first compared by plotting the time series displacement calculated by both solutions. Figure 5-2 shows this comparison. The solutions appear very similar, but the instantaneous wall displacement is not expected to match. Because of the chaotic nature of turbulence, even very small changes in the boundary conditions or locations can lead to large changes in instantaneous values in the flow field, especially after a long time. This would be expected to in turn cause changes in the instantaneous values of the structural solution which are illustrated in Figure 5-2. A more important measure of the difference between the two methods is to compare statistical values by looking at $\delta^{\prime}, V^{\prime}$, and $A^{\prime}$. The values for these dependant variables were determined by averaging over four locations on the pipe wall (top, bottom, left, and right) halfway between the clamped ends. The values are shown in Table 5-1.

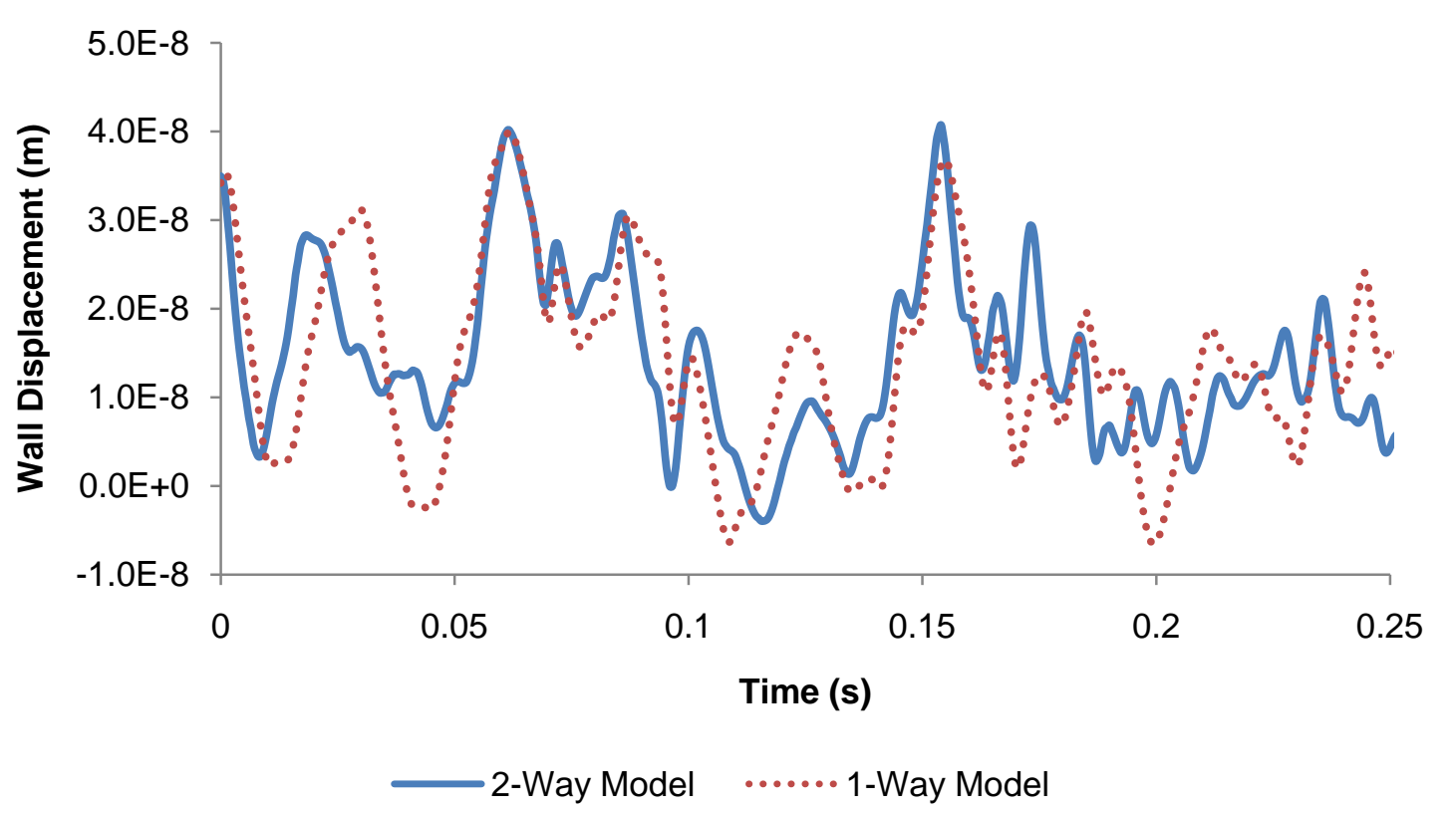

Figure 5-2 Coupling technique comparison: Time series displacement 
Table 5-1 Coupling technique comparison summary

\begin{tabular}{|c|c|c|c|}
\hline Coupling Technique & $\delta^{\prime}(\mathrm{nm})$ & $V^{\prime}(\mu \mathrm{m} / \mathrm{s})$ & $A^{\prime}\left(\mathrm{m} / \mathrm{s}^{2}\right)$ \\
\hline One-Way & 9.77 & 2.89 & 0.00349 \\
\hline Two-Way & 10.3 & 2.9 & 0.00340 \\
\hline \% Difference & $5.1 \%$ & $0.3 \%$ & $2.6 \%$ \\
\hline
\end{tabular}

The maximum relative difference between the two techniques occurs for the standard deviation of wall displacement at just over 5\%. Because the difference here between the two techniques is small negligible relative to the expected differences from investigated parameter variation, the use of the much faster one-way model is justified. 


\section{Results}

The results presented in this chapter were obtained by using the models and following the procedures described in chapters 3 through 5. This results chapter first describes the general behavior of both the long and short pipe models. The dimensionless variables used to characterize and quantify the pipe behavior are then introduced. The specific effects of each of the dimensionless variables are presented. This chapter also presents the complete functional relationships between the independent and dependant variables for both the long and short pipe which is the most important result of this work. Finally, the numerical model results obtained here are compared to available experimental data from other researchers.

\subsection{General Behavior}

Because the external forces applied to the structural model come from the wall pressures determined using the fluid model, it is useful to see how the wall pressure fluctuations are affected by the fluid solver parameters. Figure 6-1 displays the relationship between the fluid velocity (which was changed to produce the various flow solutions) and the standard deviation of wall pressure fluctuations, $P^{\prime}$, averaged over the entire pipe wall. Also shown on this plot is the average wall shear stress, $\tau_{w}$. The pressure fluctuations can be fit with a power law curve with $R^{2}=0.9997$ indicating that 
they increase with $U^{1.73}$. This scales very similarly with the average wall shear stress which increases with $U^{1.78}$, indicating that the wall shear is likely the driving factor in determining the level of the pressure fluctuations. The values of wall shear stress determined in these simulations are those caused by a smooth wall. Rough pipe walls cause a slightly different scaling of wall shear with velocity. For high Reynolds number flows though rough-walled pipes, the flow is said to be wholly turbulent, and the wall shear stress becomes proportional to $U^{2}$, where $U$ is the average fluid velocity. The numerical simulation relation of $U^{1.78}$ is for smooth walls where a wholly turbulent condition never exists.

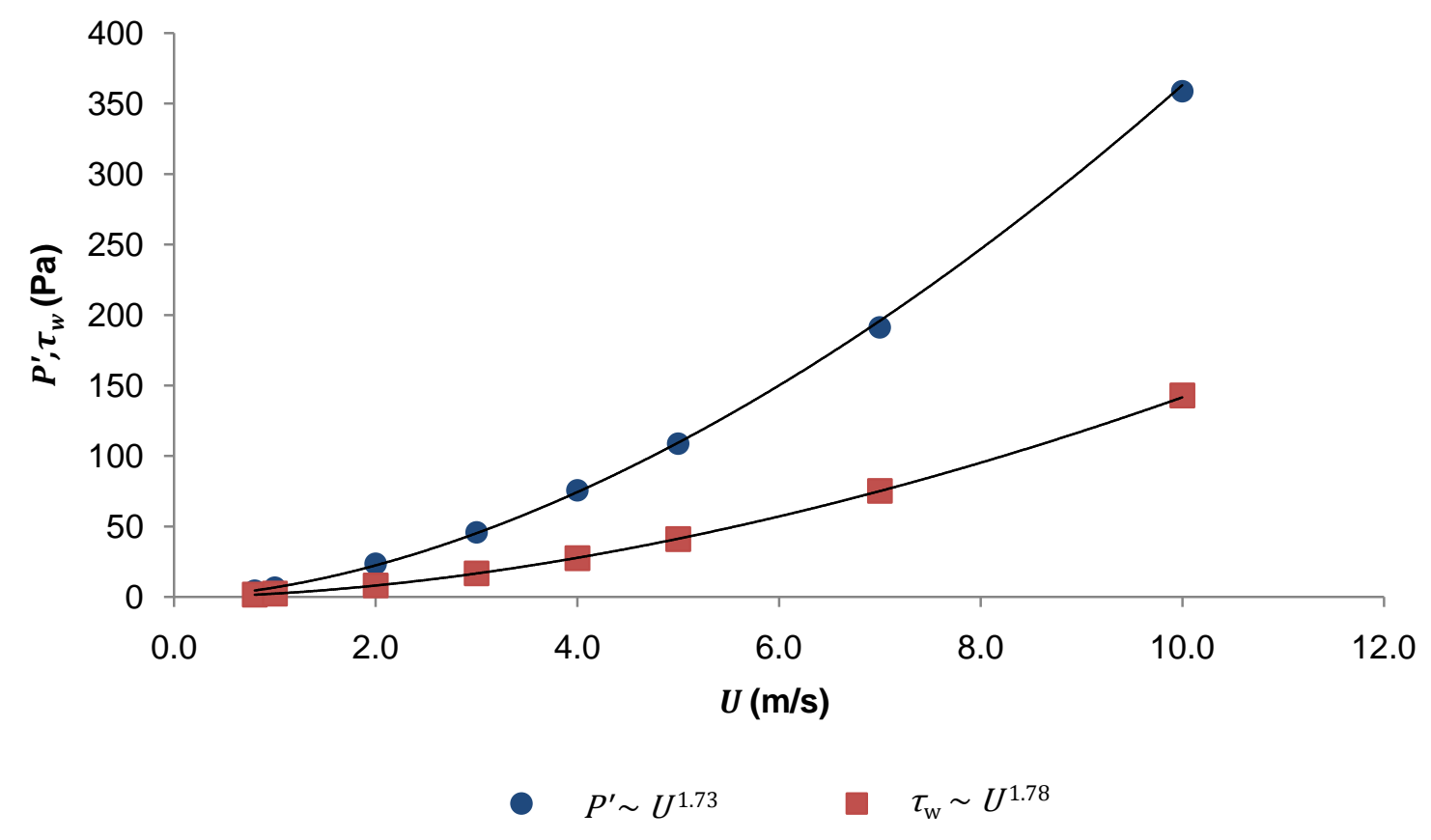

Figure 6-1 $\quad P^{\prime}$ and $\tau_{\mathrm{w}}$ as functions of average fluid velocity in a pipe having $D=$ $0.1015 \mathrm{~m}$ with water as the working fluid. 
The frequency content of the wall pressure from the fluid solution gives some indication of how energy is transferred from the fluid to the pipe. Turbulent pressure fluctuations have a wide frequency range of energy content. The spectral density (magnitude of pressure fluctuations plotted versus frequency, $\mathrm{f}$, in $\mathrm{Hz}$ ) of the pressure fluctuations from the $10 \mathrm{~m} / \mathrm{s}$ fluid solution averaged over the entire wall is shown in Figure 6-2. Experimental turbulent power spectra exhibit a classic energy cascade indicated by an amplitude decay with $f^{-5 / 3}$. The pressure spectrum from the LES simulations follows this classical behavior. The pressure fluctuations force the pipe wall at this entire range of frequencies, so the pipe is expected to respond with similar frequency content. The short pipe and long pipe display fundamentally different behaviors, partly because the short pipe analysis does not include dynamic structural effects.

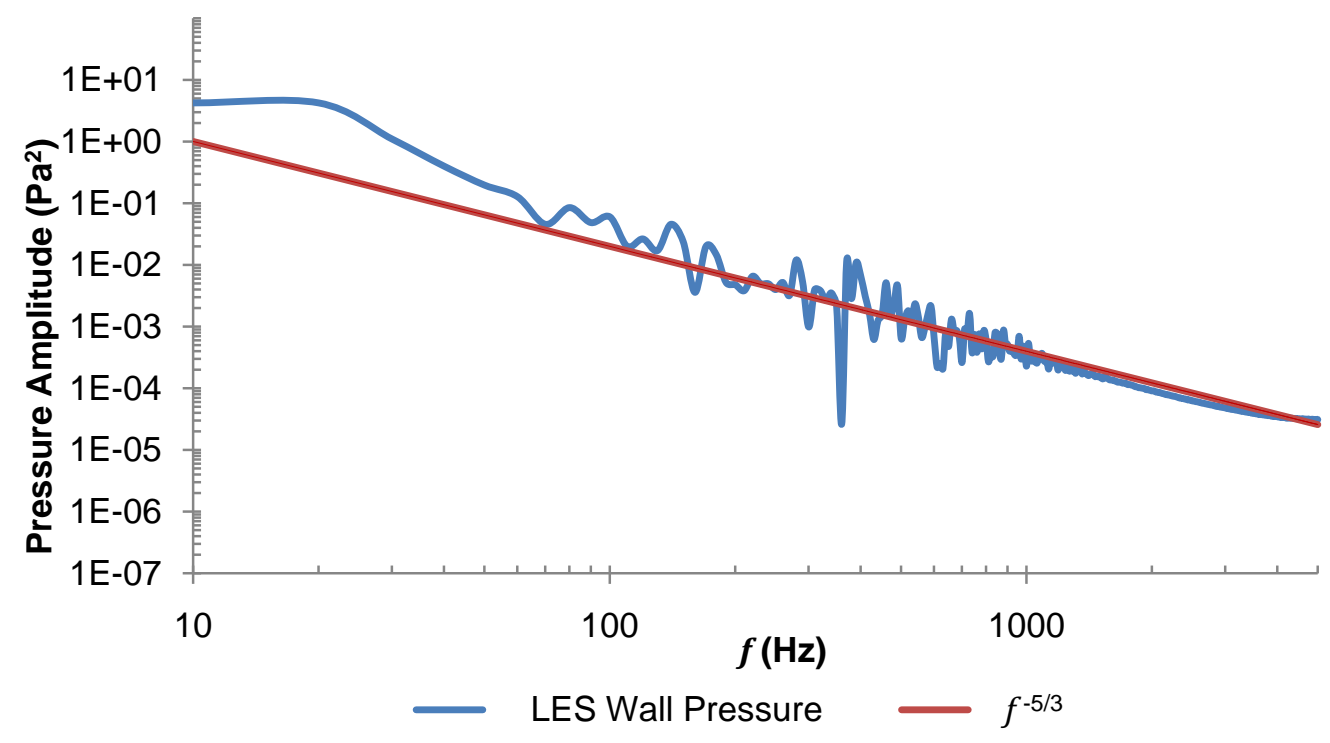

Figure 6-2 Wall pressure fluctuations as a function of frequency for the $10 \mathrm{~m} / \mathrm{s}$ flow solution 


\subsubsection{Short Pipe}

The short pipe has very high natural frequencies and is stiff enough to respond immediately to the changes in pressure from the fluid solution. Because the pipe wall responds so quickly, a full transient analysis of the short pipe was found to yield the same results as a static analysis. The time series wall displacement is taken from four nodes (top, bottom, front and back) situated around the domain, halfway between the inlet and outlet. The frequency content of the displacement at these four locations is averaged and presented in Figure 6-3. There is some noise because of the relatively short (1/10 s) sample and averaging over only four nodes, but the trend is clear. The pipe wall displacement exhibits nominally the same characteristic frequency content as the wall pressure, but with reduced response to high frequencies. This may be due to the fact that the pipe wall averages out the smallest spatial pressure variations that correspond to the highest frequency fluctuations.

Another behavior of the short pipe is its tendency to respond to local pressure variations, undergoing shell-type deformations. Rather than large portions of the pipe moving together as in bending, small areas of the surface deflect relative to adjacent areas producing an unordered pattern of valleys and peaks. Figure 6-4 shows an image of the short pipe model at settings of $U=10 \mathrm{~m} / \mathrm{s}, D=0.1015 \mathrm{~m}, t=3 \mathrm{~mm}, E=3.7 \mathrm{GPa}$, with exaggerated deflection and color contours of the displacement vector magnitude. This localized deflection was observed to dominate the short pipe motion in all of the simulations performed with this model. 


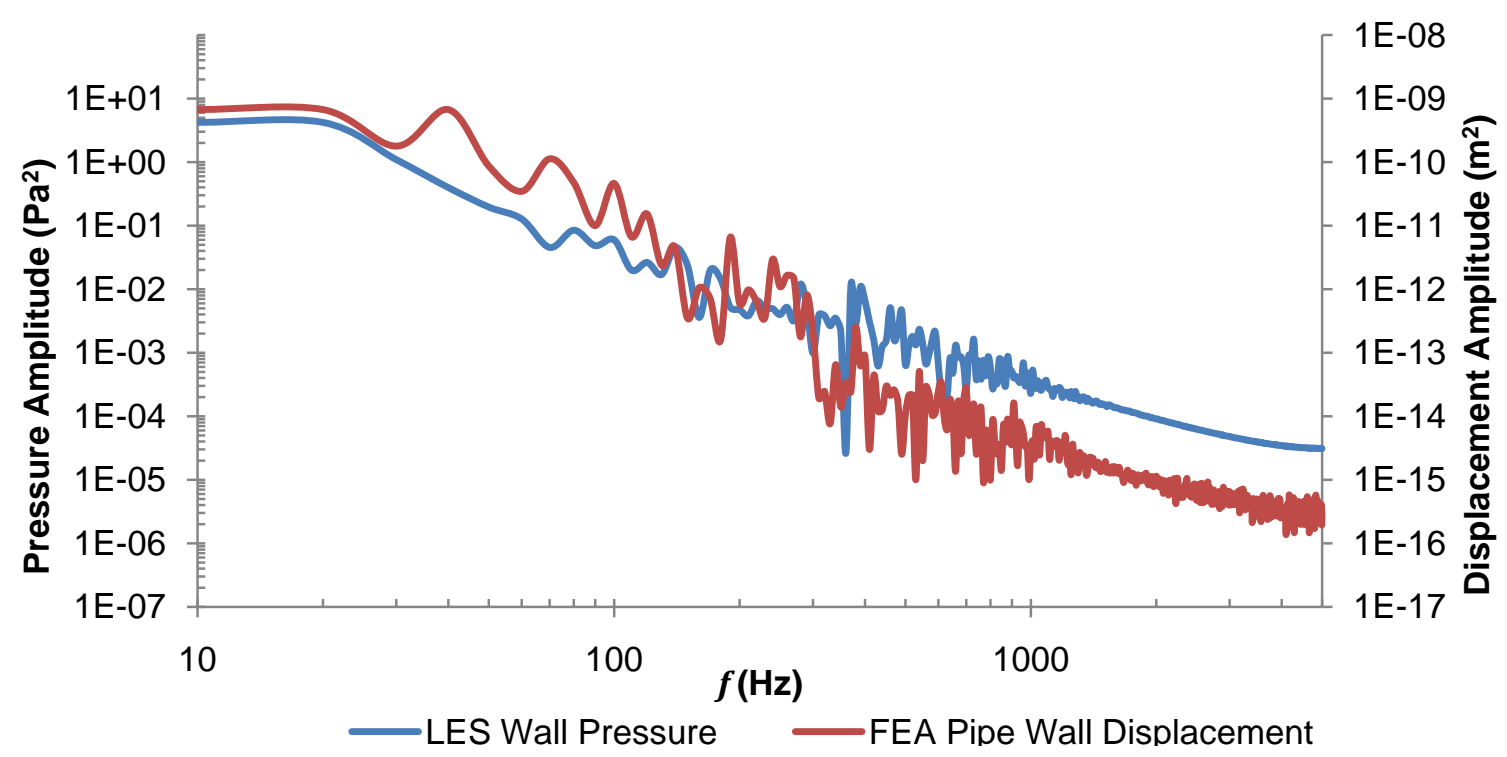

Figure 6-3 Short pipe pressure and wall displacement as a function of frequency for $U=10 \mathrm{~m} / \mathrm{s}, D=0.1015 \mathrm{~m}, t=3 \mathrm{~mm}, E=3.7 \mathrm{GPa}$

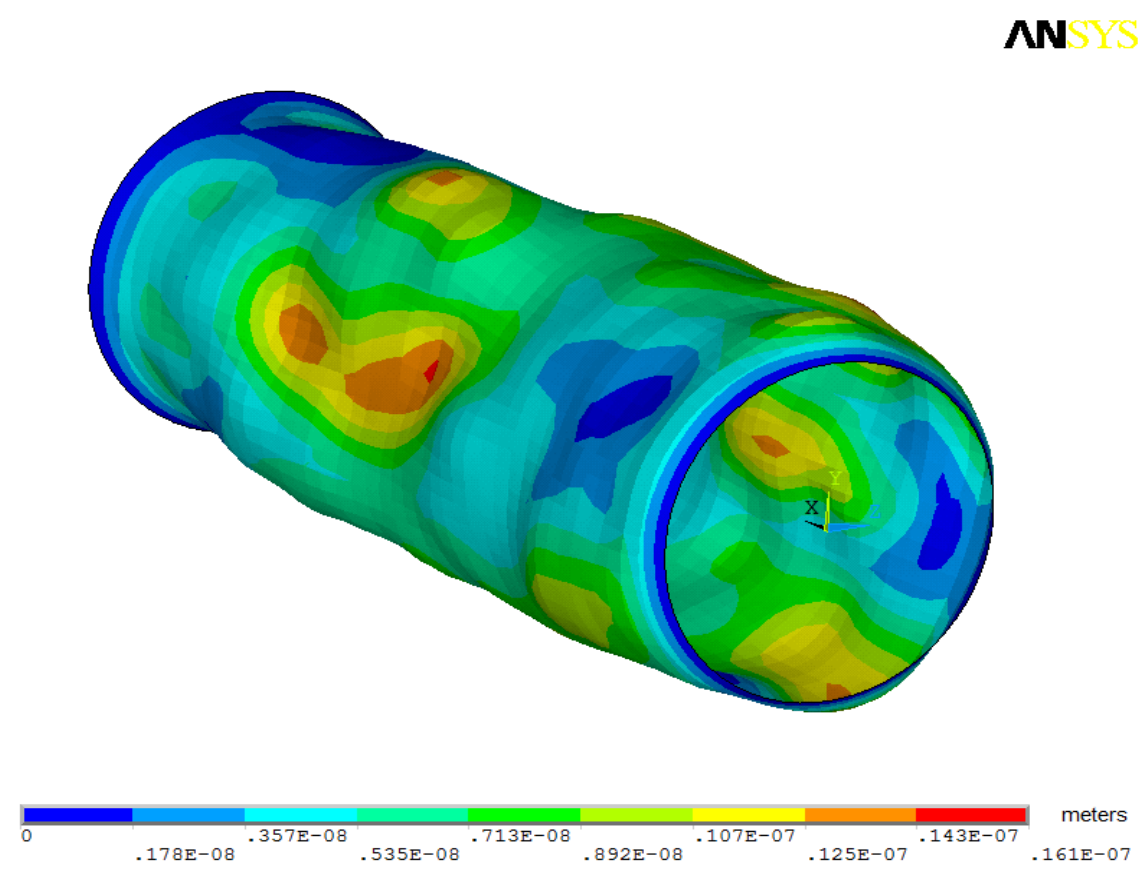

Figure 6-4 Short pipe surface deflection pattern for $U=10 \mathrm{~m} / \mathrm{s}, D=0.1015 \mathrm{~m}, t=$ $3 \mathrm{~mm}, E=3.7 \mathrm{GPa}$ 


\subsubsection{Long Pipe}

The long pipe model has lower natural frequencies and is not stiff enough to respond instantaneously to the changing pressure load. This inertial effect required the use of a full transient analysis. Due to the inclusion of inertial effects in the long pipe model, the pipe responds naturally better to certain frequencies than others. The effect is to amplify the pipe wall motion in certain bands of the broad spectrum over which it is excited by the fluctuating pressure. This effect is illustrated in Figure 6-5 which shows the spectral density of the pressure fluctuations overlaid on the spectral density of the pipe wall displacement response, with vertical bands indicating natural frequencies predicted by a modal analysis. As seen with the short pipe frequency response, the long pipe displacement spectrum has the same characteristic roll-off with increasing frequency, including reduced response at the highest frequencies. However, the long pipe also has a clear region of enhanced response which appears to coincide with the natural frequencies determined through a modal analysis, although significant noise prevents observation of any clearly defined peaks.

Any local fluctuations along the wall of the long pipe tend to be small relative to the pronounced large scale bending motions that dominate its response. The pipe tends to respond to an overall net force imbalance that induces deflections similar to those experienced by a beam subjected to a distributed load. Figure 6-6 shows an image of the deformed long pipe at settings of $U=10 \mathrm{~m} / \mathrm{s}, D=0.1015 \mathrm{~m}, t=2 \mathrm{~mm}, E=21 \mathrm{GPa}, \rho_{e q}$

$=3000 \mathrm{~kg} / \mathrm{m}^{3}, \beta=0.001$ with exaggerated displacement scaling and color contours of displacement magnitude. This bending-beam type of deflection is typical of almost any time step in all of the long pipe solutions. 


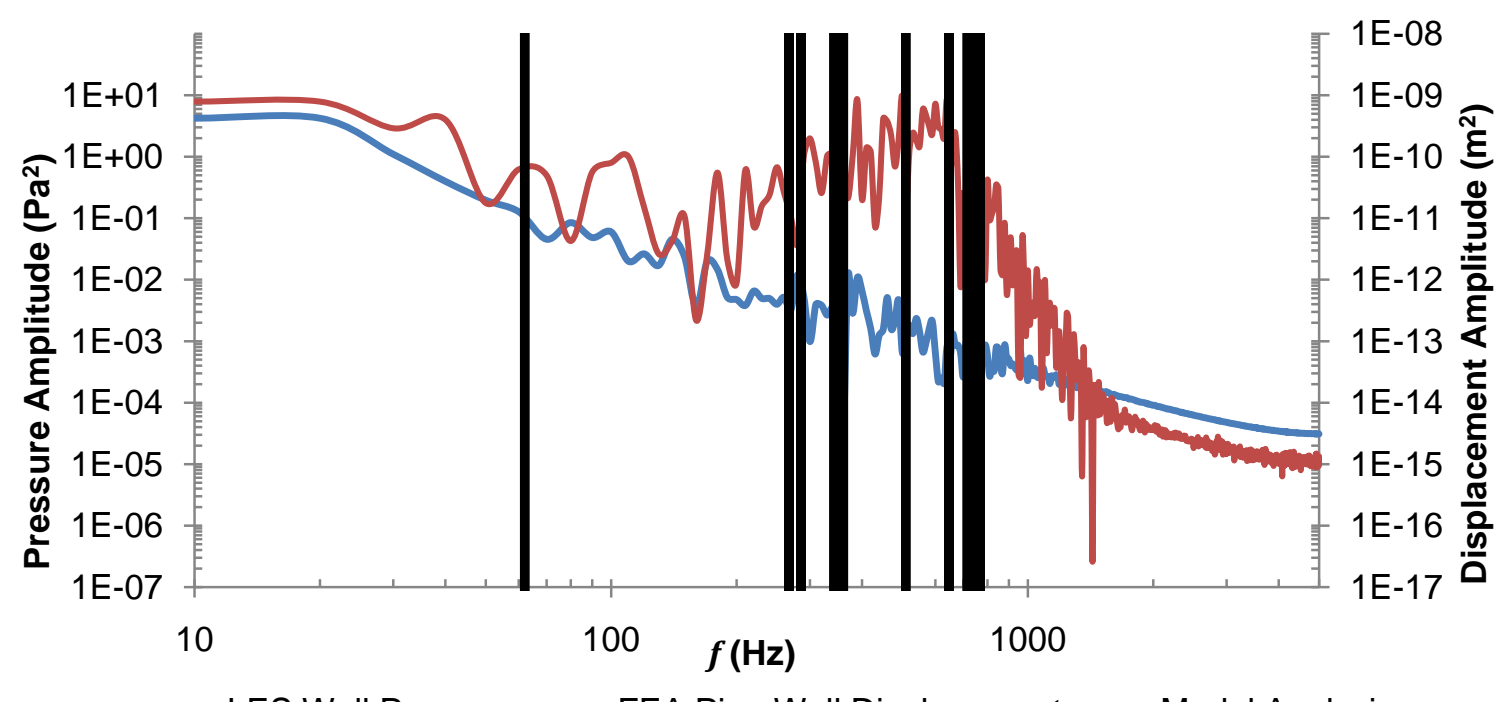

Figure 6-5 Long pipe pressure and wall displacement as a function of frequency content for $U=10 \mathrm{~m} / \mathrm{s}, D=0.1015 \mathrm{~m}, t=2 \mathrm{~mm}, E=21 \mathrm{GPa}, \rho_{\mathrm{eq}}=3000 \mathrm{~kg} / \mathrm{m}^{3}, \beta=$ 0.001. Also shown are pipe natural frequencies predicted using a modal analysis
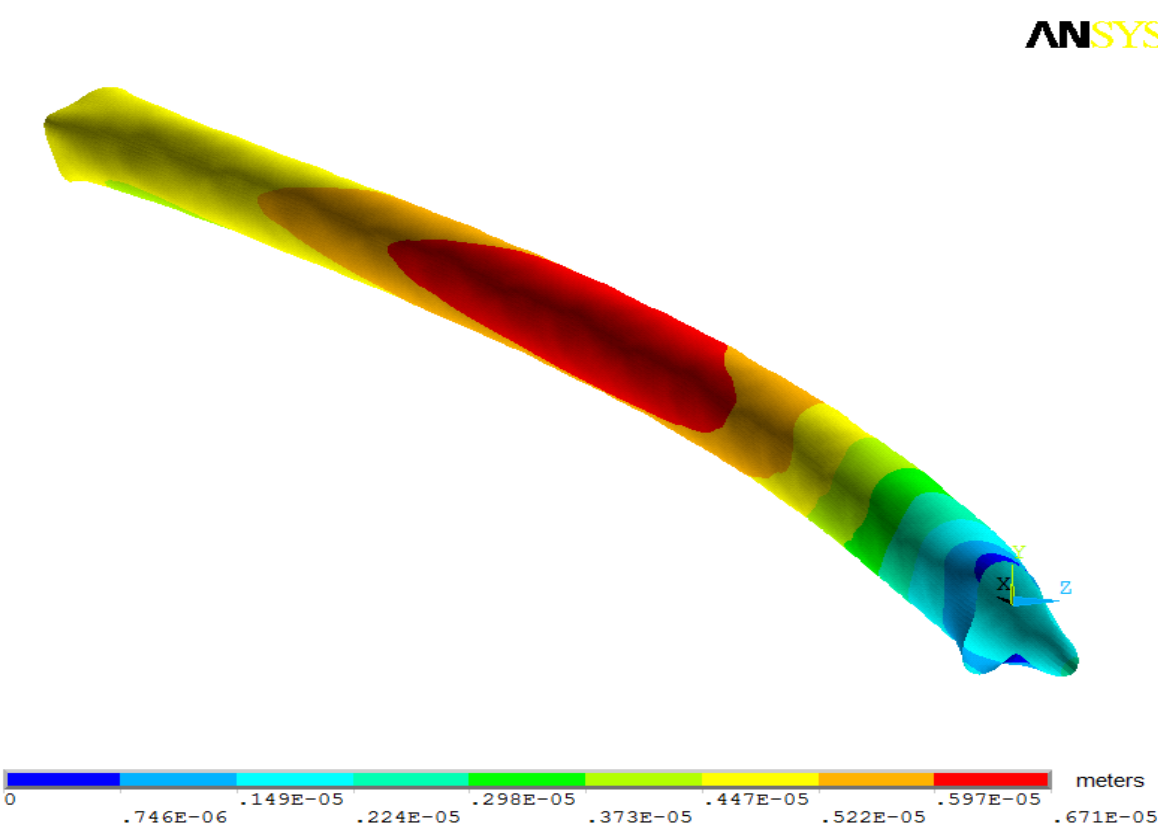

Figure 6-6 Long pipe wall displacement pattern for $U=10 \mathrm{~m} / \mathrm{s}, D=0.1015 \mathrm{~m}, t=$ $2 \mathrm{~mm}, E=21 \mathrm{GPa}, \rho_{\mathrm{eq}}=3000 \mathrm{~kg} / \mathrm{m}^{3}, \beta=0.001$ 


\subsection{Non-Dimensionalization}

Dimensional analysis was used to reduce the total number of important variables needed to characterize the problem. The dimensional analysis also gives the results in more generalized form, convenient for comparisons with other work. A complete nondimensional set of variables accounts for and represents all of the original dependant and independent variables of interest. The dimensionless forms of each of the dependant variables are defined in Table 6-1.

\section{Table 6-1 Dimensionless dependant variables}

\begin{tabular}{|c|c|c|}
\hline $\begin{array}{c}\text { Dimensionless } \\
\text { Variable }\end{array}$ & Definition & Description \\
\hline$\delta^{*}$ & $\frac{\delta^{\prime}}{D}$ & $\begin{array}{c}\text { Ratio of standard deviation of pipe wall } \\
\text { displacement to internal diameter }\end{array}$ \\
\hline$V^{*}$ & $\frac{V^{\prime}}{U}$ & $\begin{array}{c}\text { Ratio of standard deviation of pipe wall velocity to } \\
\text { average fluid velocity }\end{array}$ \\
\hline$A^{*}$ & $\frac{A^{\prime} t}{U^{2}}$ & Dimensionless pipe wall acceleration \\
\hline
\end{tabular}

Before defining the dimensionless form of the independent variables it is useful to introduce two new variables. The first represents a characteristic frequency of the fluid flow and is defined as:

$$
\omega_{c}=\frac{U}{D}
$$

which indicates the lowest frequency of coherent structures expected to be present in the turbulent flow field [28]. The second variable was actually mentioned briefly in chapter 4. It is proportional to the fundamental natural frequency of the pipe in bending. It is 
defined as:

$\omega_{n}=\sqrt{\frac{E}{\rho_{e q}}} \frac{D}{L^{2}}$

and is related to the lowest expected natural frequency of a long pipe structure [29].

Using these two characteristic frequencies the dimensionless independent variables are defined in Table 6-2. The total number of independent variables influencing the pipe motion has been reduced from the original $9\left(\mu, \rho_{f}, U, D, t, L, \rho_{e q}, E\right.$, and $\left.\beta\right)$, to the six dimensionless independent variables.

Table 6-2 Dimensionless independent variables

\begin{tabular}{|c|c|c|}
\hline $\begin{array}{c}\text { Dimensionless } \\
\text { Variable }\end{array}$ & Definition & Description \\
\hline$R e_{D}$ & $\frac{\rho_{f} U D}{\mu}$ & Ratio of fluid inertial forces to viscous forces \\
\hline$t^{*}$ & $\frac{t}{D}$ & Ratio of pipe wall thickness to diameter \\
\hline$L^{*}$ & $\frac{L}{D}$ & Ratio of pipe length to diameter \\
\hline$\rho^{*}$ & $\rho_{e q}$ & Ratio of total mass to fluid mass \\
\hline$\omega^{*}$ & $\frac{\omega_{n}}{\omega_{c}}$ & Ratio of pipe frequencies to fluid frequencies \\
\hline$\zeta$ & $\beta \omega_{n}$ & Damping ratio \\
\hline
\end{tabular}




\subsection{Specific Variable Effects}

The first order effects of each of the independent variables are presented for both the long and short pipe models. Each of the dimensionless dependent variables was found to have a power-law dependency on each of the dimensionless independant variables. The power law dependencies only apply over the range of variables considered.

\subsubsection{Short Pipe}

Because the structural short pipe model was solved without transient effects, and because it had few material properties defined, it is affected by only some of the independent variables. Only those variables exerting influence on the short pipe are presented here. Each of the plots include three sets of results indicating the effects of a particular independent variable on the three dependant variables, $\delta^{*}, V^{*}$, and $A^{*}$. Because the short pipe model does not account for transient structural effects it does not make sense to define a natural pipe frequency, $\omega_{n}$. For this reason, instead of considering a ratio of structural and fluid frequencies, a non-dimensional stiffness variable is used in the place of $\omega^{*}$. This variable is defined as:

$$
E^{*}=\frac{E}{\rho U^{2}}
$$

and is the ratio of the elastic modulus of the pipe material to twice the dynamic pressure of the fluid. The short pipe response is then a function of three variables, namely $R e_{D}$, $t^{*}$, and $E^{*}$.

The short pipe response as a function of the Reynolds number was found by holding $E^{*}$ and $t^{*}$ constant at values of $3.71 \times 10^{6}$ and 0.0296 respectively while repeating the 
simulation at each of the eight Reynolds numbers. There is a definite trend in the data showing that all of the dependant variables are inversely related to the Reynolds number. A plot of the dependence of $\delta^{*}, V^{*}$, and $A^{*}$ on $R e_{D}$ is shown in Figure 6-7 along with a power law curve fit to each data set. The relationship here is somewhat noisy, likely due to the small number of time steps of the fluid simulations. $\delta^{*}$ was found to be proportional to $R e_{D}^{-0.26}, V^{*}$ was found to be proportional to $R e_{D}{ }^{-0.30}$, and $A^{*}$ was found to be proportional to $R e_{D}{ }^{-0.38}$. The power law curves fit to the data have average relative error values of $10.8 \%$ for $\delta^{*}, 6.3 \%$ for $V^{*}$, and $11.1 \%$ for $A^{*}$.

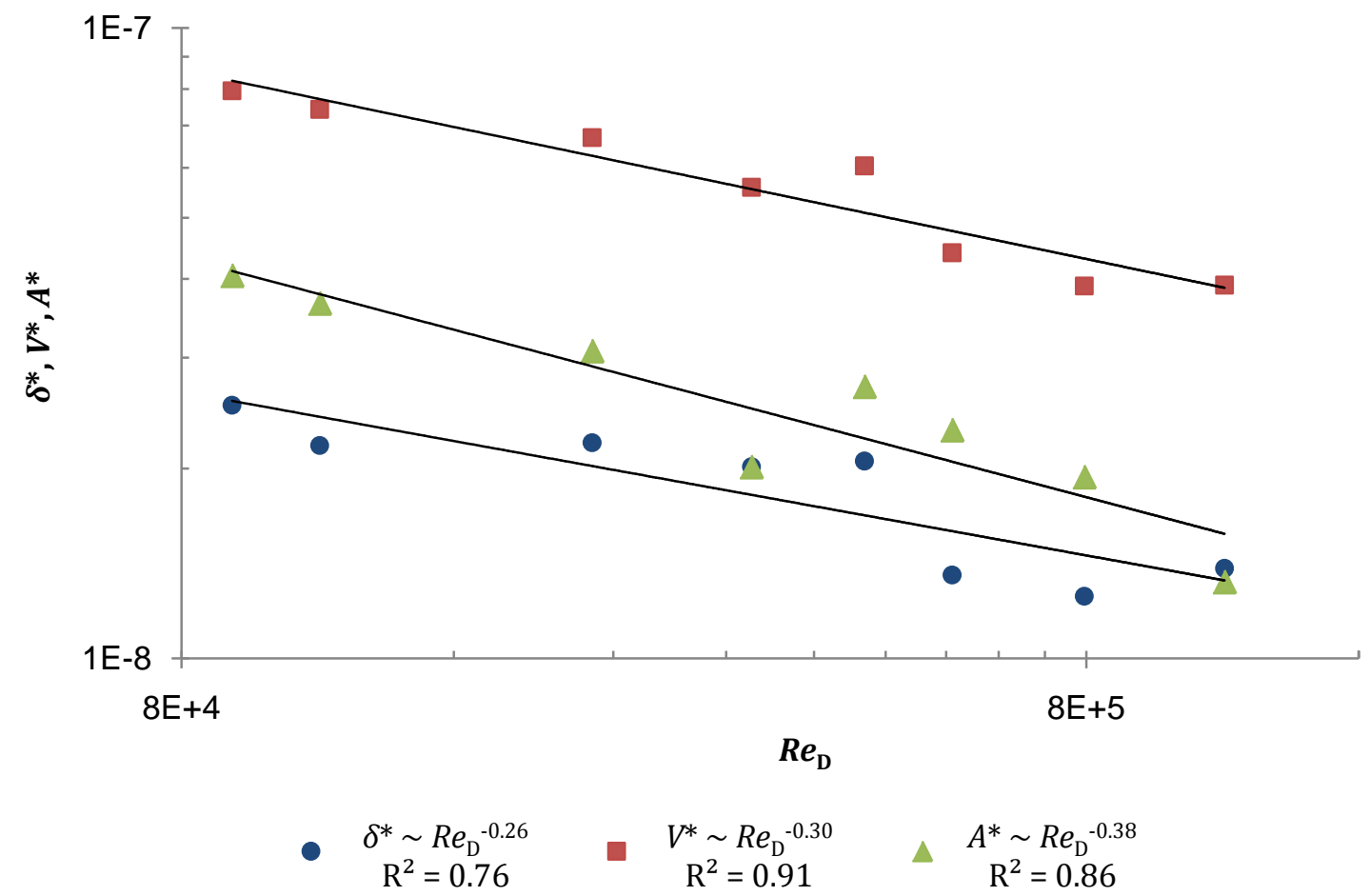

Figure 6-7 $\delta^{*}, V^{*}$, and $A^{*}$ as functions of Reynolds number for the short pipe model with $t^{*}=0.0296$ and $E^{*}=3.71 \times 10^{6}$ 
Figure 6-8 illustrates the effect of changing the thickness to diameter ratio for the short pipe model. This relationship was determined by holding the other nondimensional variables constant at $R e_{D}=1.14 \times 10^{5}$ and $E^{*}=3.71 \times 10^{6}$ while varying the thickness to diameter ratio. This plot indicates a strong inverse dependence for all of the response variables, with the data all being fit very well by power law curves. There is very little noise here because all of the simulations used the same fluid solution. $\delta^{*}$ was found to be proportional to $t^{*-2.05}, V^{*}$ was found to be proportional to $t^{*-1.96}$, and $A^{*}$ was found to be proportional to $t^{*-0.90}$. The average relative error values of the fit curves are $3.8 \%$ for $\delta^{*}, 1.9 \%$ for $V^{*}$, and $1.4 \%$ for $A^{*}$.

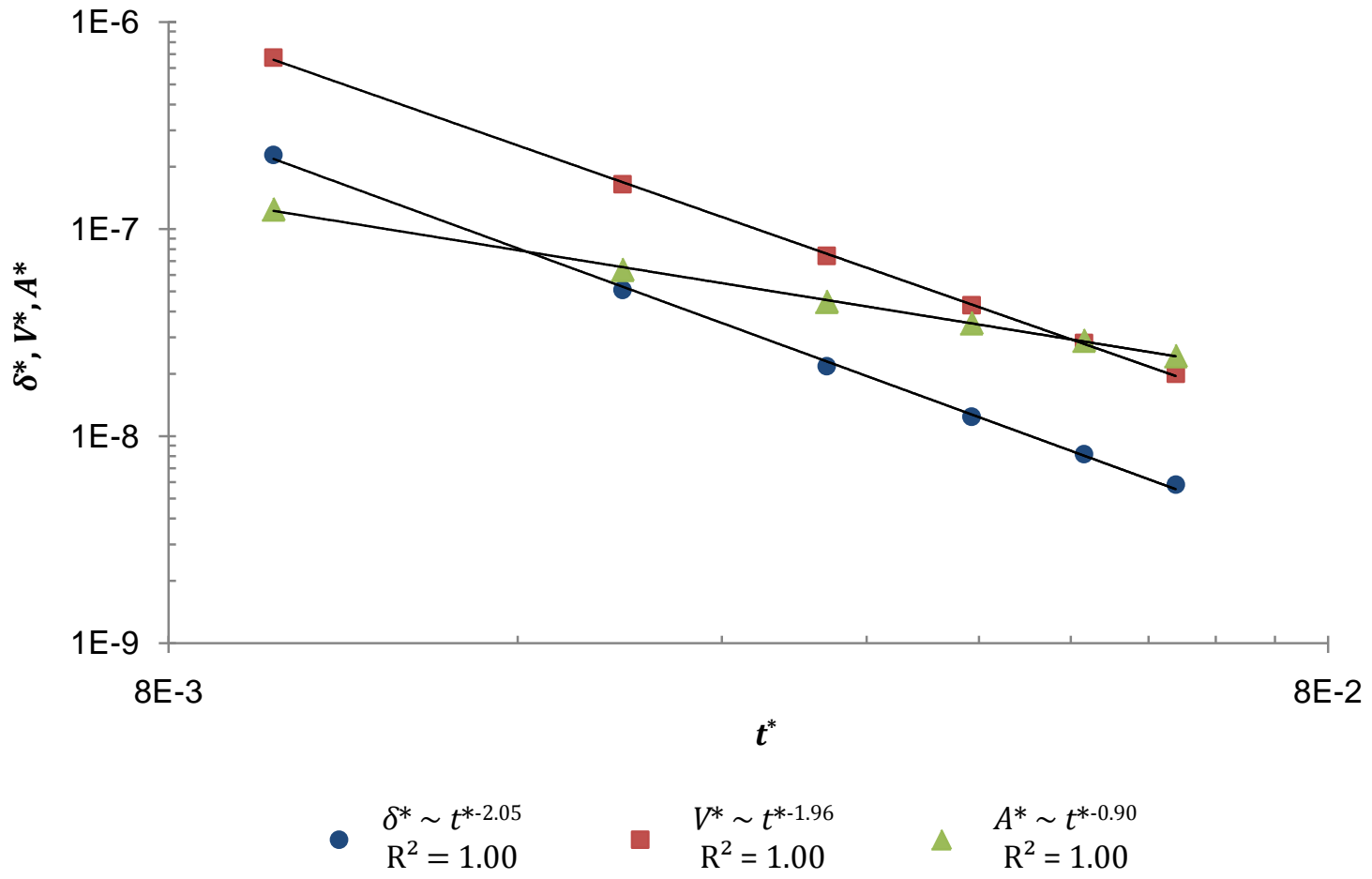

Figure 6-8 $\delta^{*}, V^{*}$, and $A^{*}$ as functions of $t^{*}$ for the short pipe model with $R e_{\mathrm{D}}=$ $1.14 \times 10^{5}$ and $E^{*}=3.71 \times 10^{6}$ 
Figure 6-9 indicates that all of the dependant variables are inversely proportional to $E^{*}$. The simulations providing these results were all carried out using the same fluid solution at $R e_{D}=4.55 \times 10^{5}$, and constant thickness ratio of $t^{*}=0.0296$. The inverse proportionality is expected since the static deflection of any element is inversely proportional to the modulus of elasticity. Neglecting transient effects, the velocity and acceleration of the pipe wall are just the first and second derivatives of the wall deflection with respect to time, and the inverse proportionality with E still holds for these variables. The inverse proportionality curves fit the data remarkably well with negligible relative error.

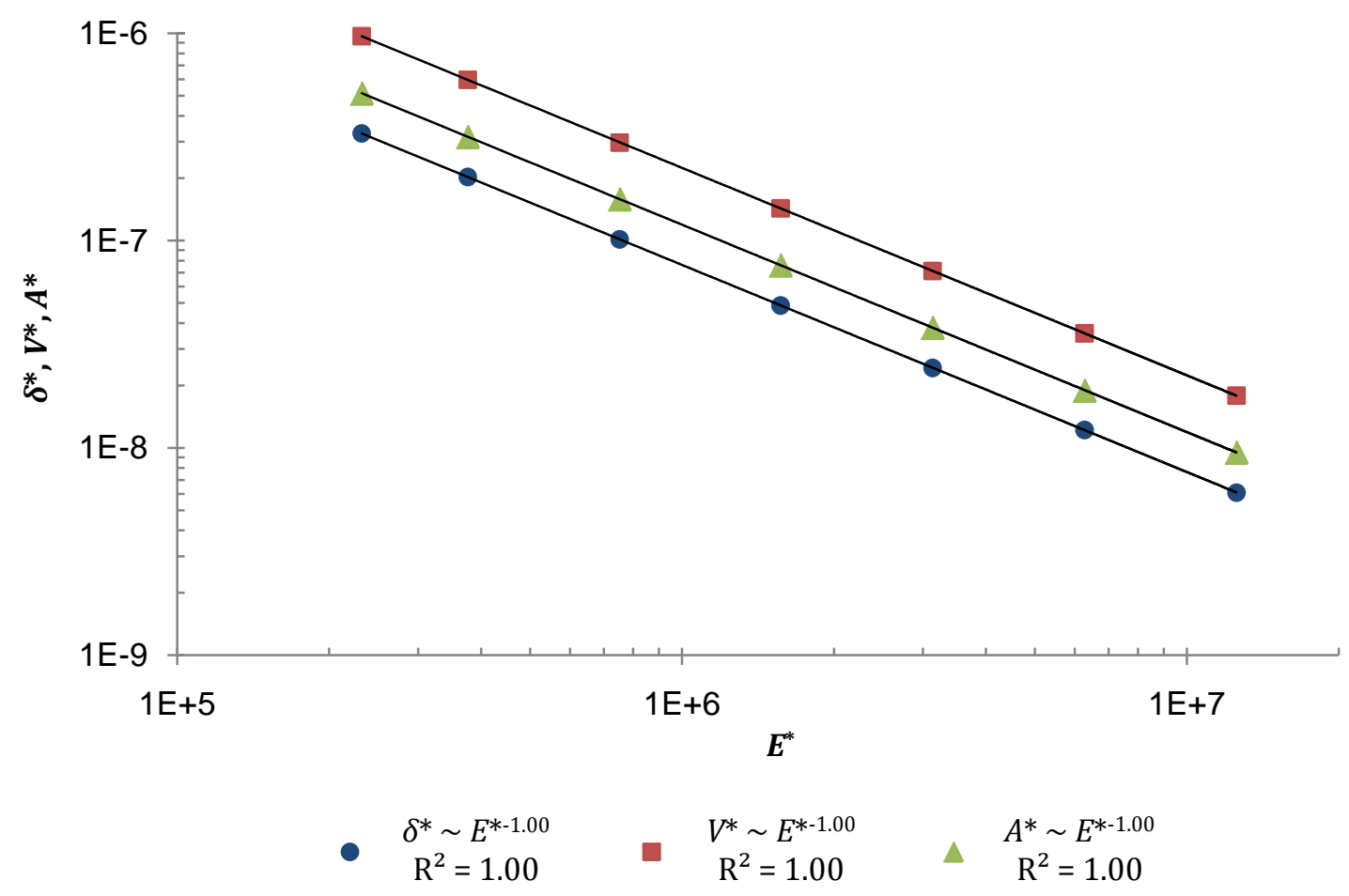

Figure 6-9 $\delta^{*}, V^{*}$, and $A^{*}$ as functions of $E^{*}$ for the short pipe model with $R e_{\mathrm{D}}=$ $4.55 \times 10^{5}$ and $t^{*}=0.0296$ 


\subsubsection{Long Pipe}

The long pipe model includes the effects of the pipe inertia and is therefore influenced by all of the dimensionless independent variables considered. The first order effects of a particular variable were found by allowing that variable to take on different values while holding all of the others constant.

The influence of Reynolds number on the long pipe response is shown in Figure 6-10. The other variables were held at $t^{*}=0.0296, L^{*}=23.6, \rho^{*}=3.01, \omega^{*}=0.473$, and $\zeta=0.047$. Like the corresponding data from the short pipe model, the long pipe Reynolds number dependence data exhibits some modest variation. Again, the trend is an inverse relationship, although for the long pipe the dependence on Reynolds number is weaker than seen for the short pipe model producing nearly flat lines on this plot. Because the Reynolds number dependence is so low, the noise is much more visible and the values of $\mathrm{R}^{2}$ for the power law curves fit to the data are lower than might be expected. The average relative error of the fit curves is $5.2 \%$ for $\delta^{*}, 6.2 \%$ for $V^{*}$, and $8.0 \%$ for $A^{*}$. The fit curves indicate that $\delta^{*}$ is proportional to $R e_{D}{ }^{-0.20}, V^{*}$ is proportional to $R e_{D}{ }^{-0.12}$, and $A^{*}$ is proportional to $R e_{D}{ }^{-0.18}$.

The thickness ratio dependence simulations for the long pipe were all carried out at $R e_{D}=1.14 \times 10^{6}, L^{*}=23.6, \rho^{*}=10.0, \omega^{*}=0.473$, and $\zeta=0.047$. The data as seen in Figure 6-11 indicates that both $\delta^{*}$ and $V^{*}$ are inversely proportional to $t^{*}$, while $A^{*}$ exhibits little dependence on $t^{*}$. Power-law curves fit the data with average relative error of $5.4 \%$ for $\delta^{*}, 3.4 \%$ for $V^{*}$, and $0.7 \%$ for $A^{*}$. The curves indicate that $\delta^{*}$ is proportional to $t^{*-1.16}, V^{*}$ is proportional to $t^{*-1.06}$, and $A^{*}$ is proportional to $t^{* 0.04}$. The pipe wall response variables' dependence on $t^{*}$ is lower for the long pipe than for the short pipe. 


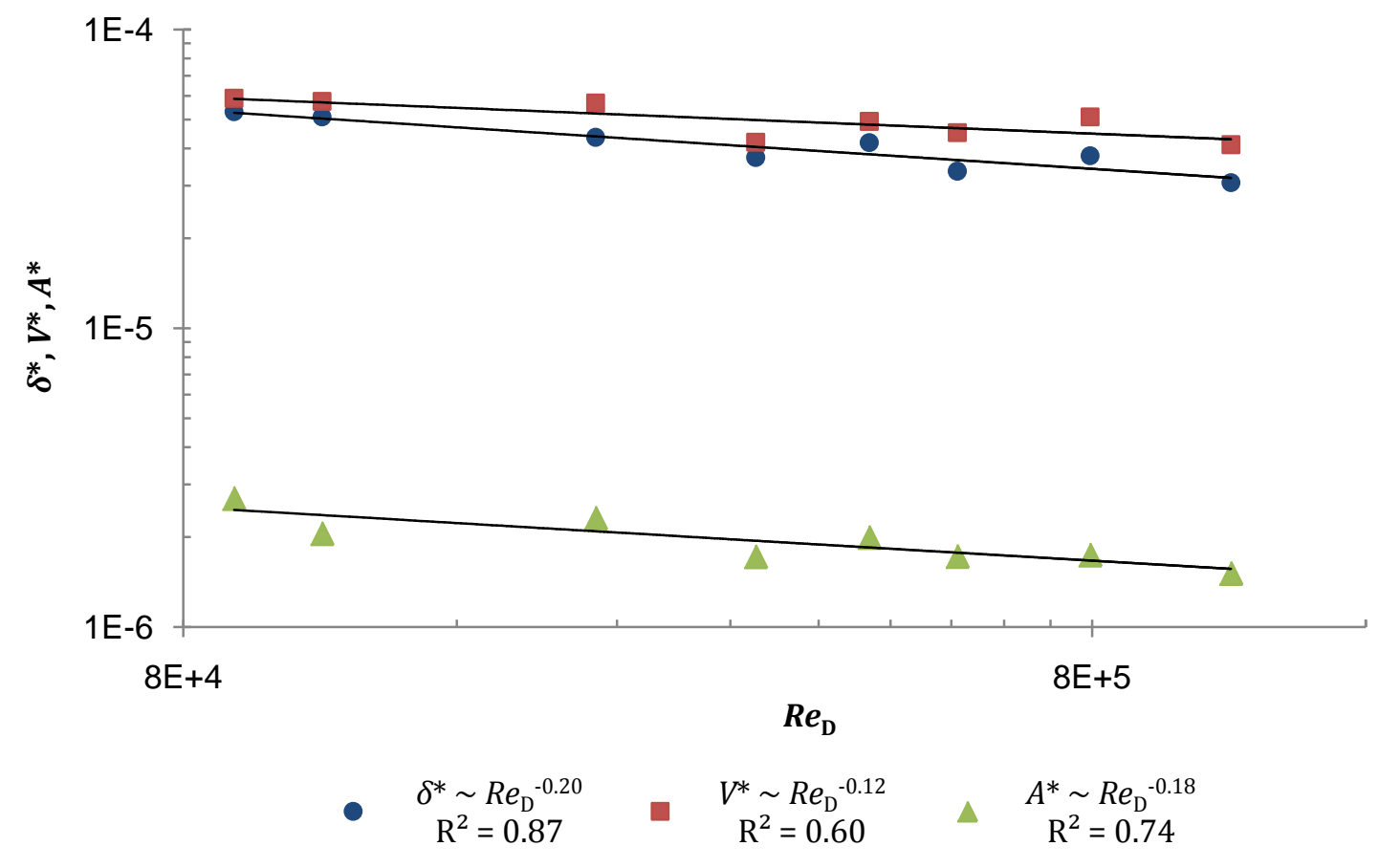

Figure 6-10 $\delta^{*}, V^{*}$, and $A^{*}$ as functions of $\operatorname{Re}_{\mathrm{D}}$ for the long pipe model with $\boldsymbol{t}^{*}=$ $0.0197, L^{*}=23.6, \rho^{*}=3.01, \omega^{*}=0.473$, and $\zeta=0.047$

This is probably due to the difference in the general nature of the pipe response between the two models. Because the short pipe responds locally to pressure fluctuations, it is highly affected by the thickness ratio which influences the spatial averaging effect on the pressure and influences the wall stiffness. The long pipe is affected by the thickness ratio only through the resulting change in stiffness.

The influence of $L^{*}$ was determined a bit differently than the other variables. As illustrated when comparing the short and long pipe models, the length ratio is involved in complex or higher order effects. For this reason, only a narrow range of length ratios is considered for first order effects. The effective length of the pipe was varied by including additional nodal degree of freedom restrictions to simulate the addition of supporting clamps on the pipe. These supports were added at two axial locations on the 


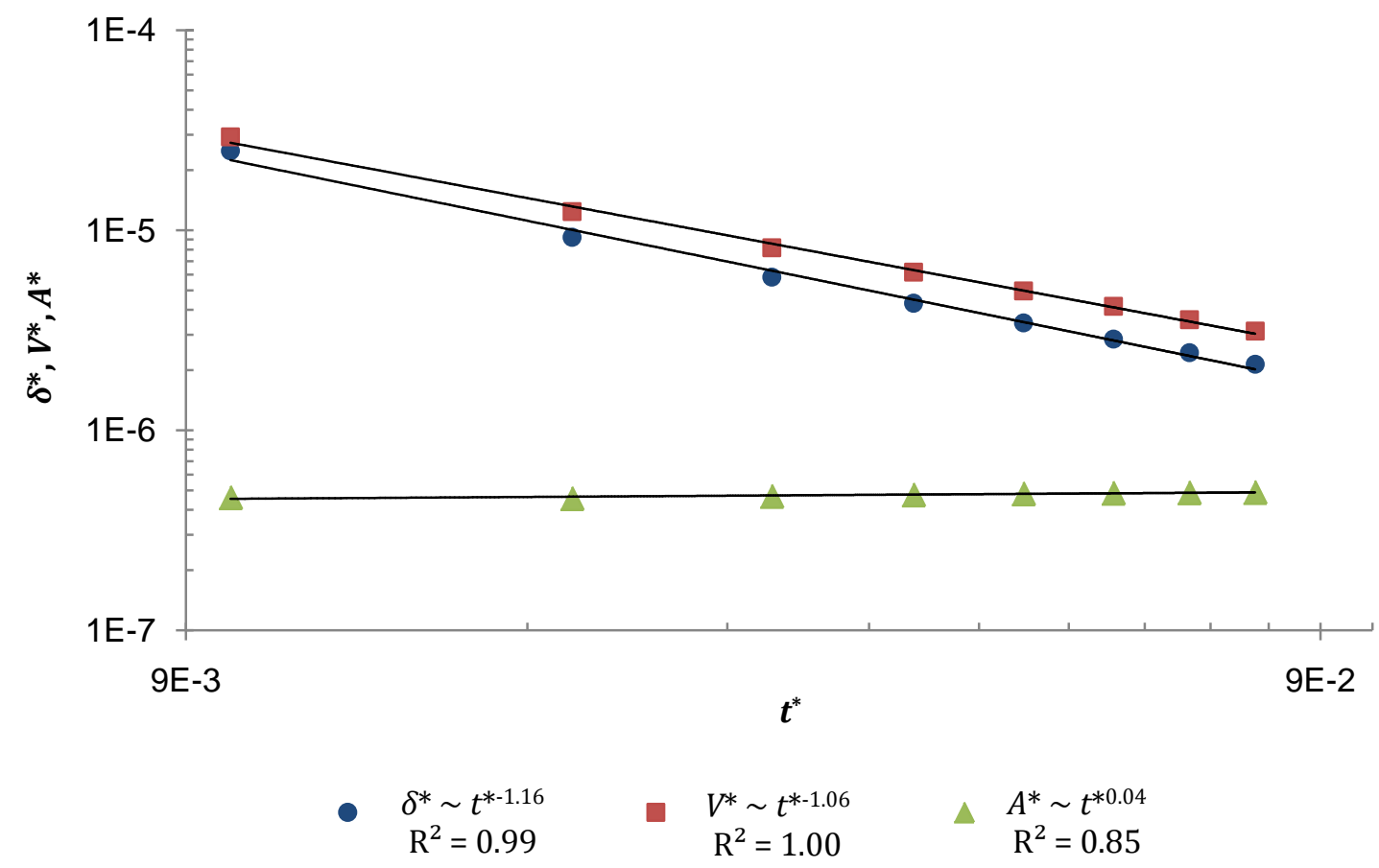

Figure 6-11 $\delta^{*}, V^{*}$, and $A^{*}$ as functions of $t^{*}$ for the long pipe model with $R e_{\mathrm{D}}=$ $1.14 \times 10^{6}, L^{*}=23.6, \rho^{*}=10.0, \omega^{*}=0.473$, and $\zeta=0.047$

pipe wall, each an equal distance in from either end of the pipe. The effective pipe length was taken as the distance between the two new support locations. The length ratio dependence, as seen in Figure 6-12, shows a slight inverse relationship for each of the dependent variables with $\delta^{*}$ being most strongly dependent on $L^{*}$, where it is proportional to $L^{*-0.72} . V^{*}$ is proportional to $L^{*-0.34}$, and $A^{*}$ is proportional to $L^{*-0.32}$. The x-axis is shown using a linear scale because of the narrow range of explored values. The other independent variables were held constant at $R e_{D}=1.14 \times 10^{6}, t^{*}=0.0197, \rho^{*}=3.01, \omega^{*}=$ 0.473 , and $\zeta=0.047$. Although the $\mathrm{R}^{2}$ values are somewhat low, the power law curves fit the data with average relative error of $3.9 \%$ for $\delta^{*}, 1.9 \%$ for $V^{*}$, and $2.4 \%$ for $A^{*}$. 


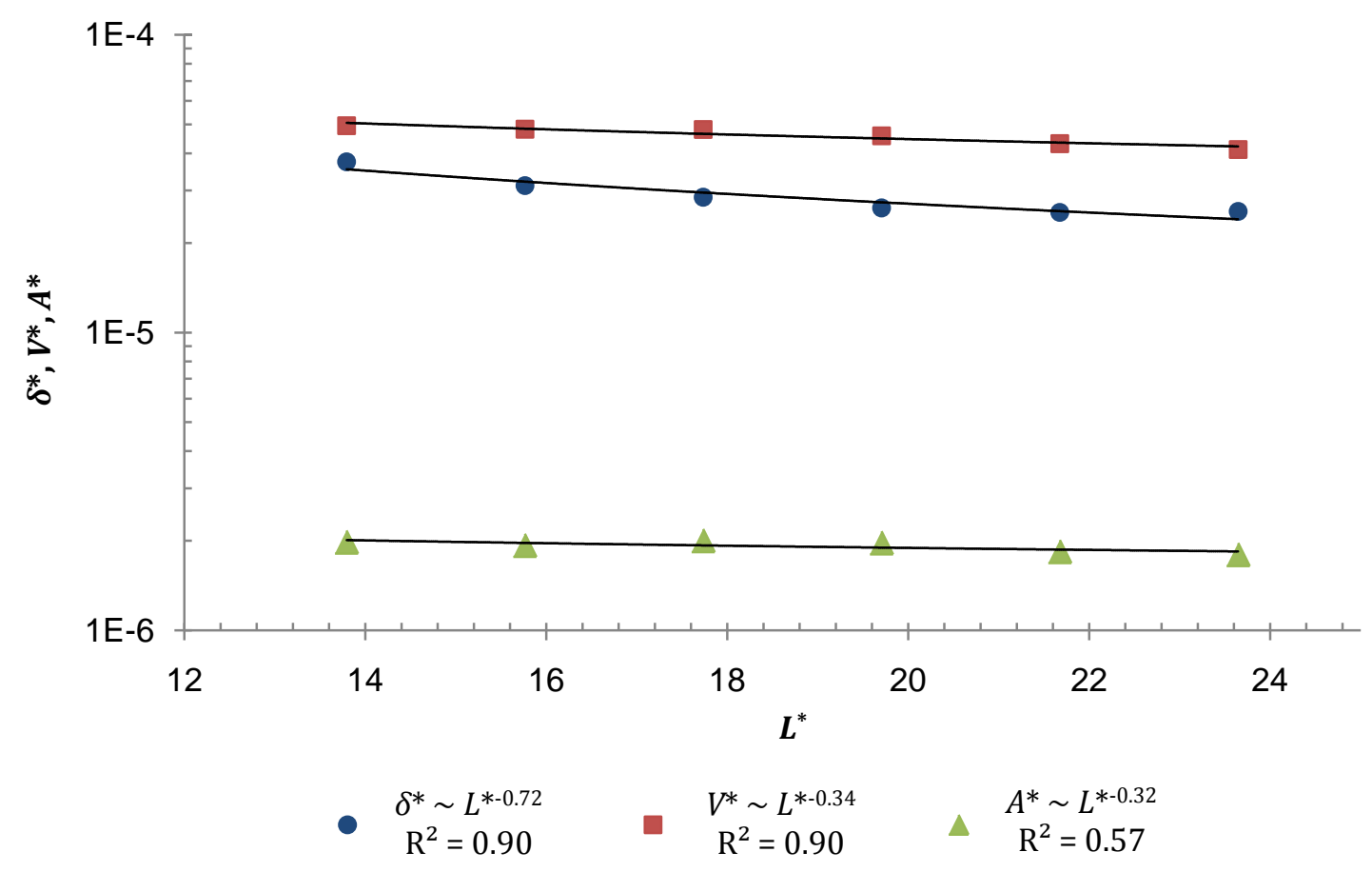

Figure 6-12 $\delta^{*}, V^{*}$, and $A^{*}$ as functions of $L^{*}$ for the long pipe model with $R e_{\mathrm{D}}=$ $1.14 \times 10^{6}, t^{*}=0.0197, \rho^{*}=3.01, \omega^{*}=0.473$, and $\zeta=0.047$

The density ratio, $\rho^{*}$, was varied while holding the other variables at $R e_{D}=$ $1.14 \times 10^{6}, t^{*}=0.0296, L^{*}=23.6, \omega^{*}=0.473$, and $\zeta=0.047$. The dependence of the pipe wall response on the density ratio is shown in Figure 6-13. The relationship is a perfect inverse proportionality for each dependant variable with negligible relative error in the inverse fit.

The effects of $\omega^{*}$ were tested while holding the other independent variables constant at $R e_{D}=1.14 \times 10^{6}, t^{*}=0.0296, L^{*}=23.6, \rho^{*}=12.0$, and $\zeta=0.047$. As illustrated in Figure 6-14, $\delta^{*}$ is proportional to $\omega^{*-1.81}, V^{*}$ is proportional to $\omega^{*-0.67}$, and $A^{*}$ is proportional to $\omega^{* 0.10}$. Power-law curves fit the data with relative error of $7.9 \%$ for $\delta^{*}, 12.5 \%$ for $V^{*}$, and $4.5 \%$ for $A^{*}$. 


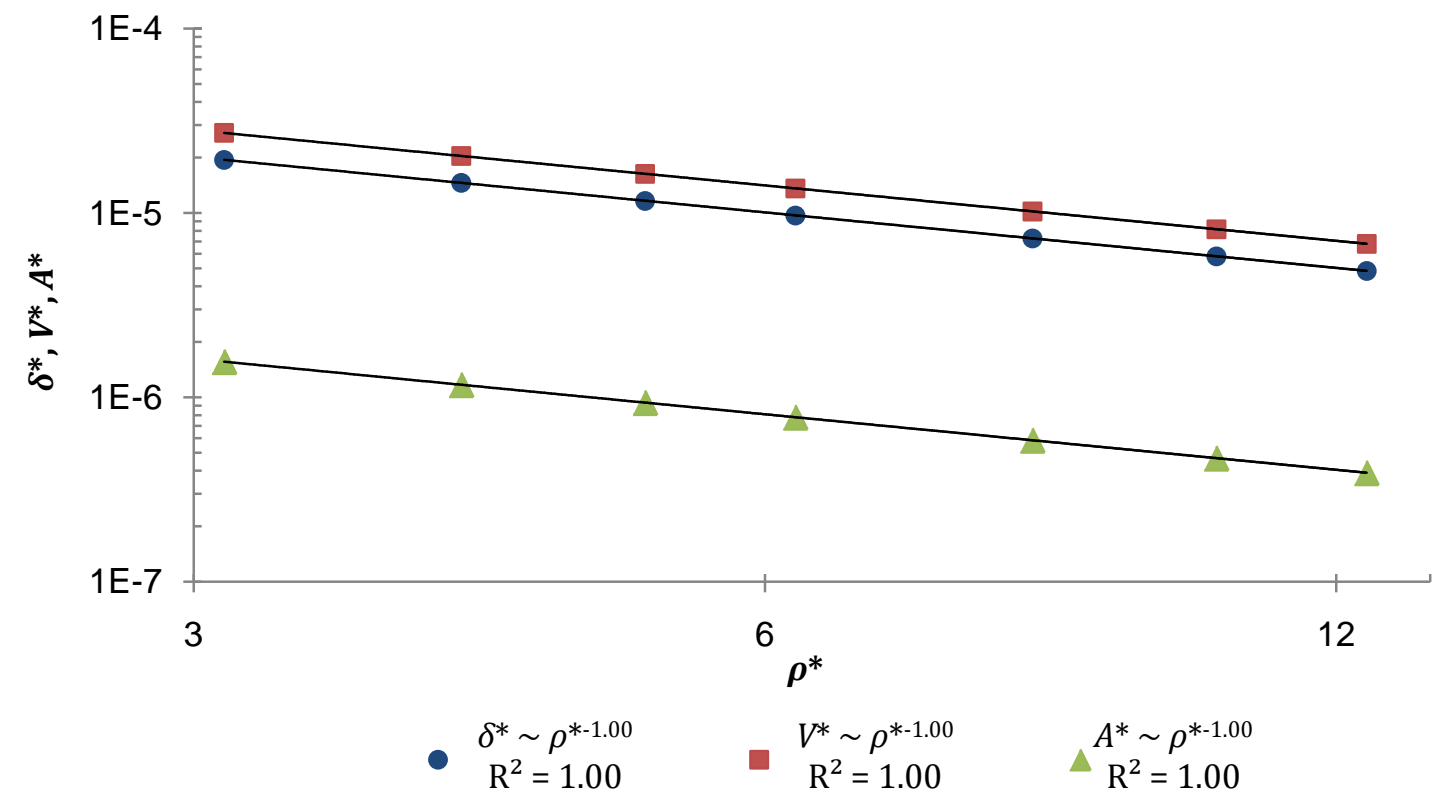

Figure 6-13 $\delta^{*}, V^{*}$, and $A^{*}$ as functions of $\rho^{*}$ for the long pipe model with $R e_{\mathrm{D}}=$ $1.14 \times 10^{6}, t^{*}=0.0296, L^{*}=23.6, \omega^{*}=0.473$, and $\zeta=0.047$

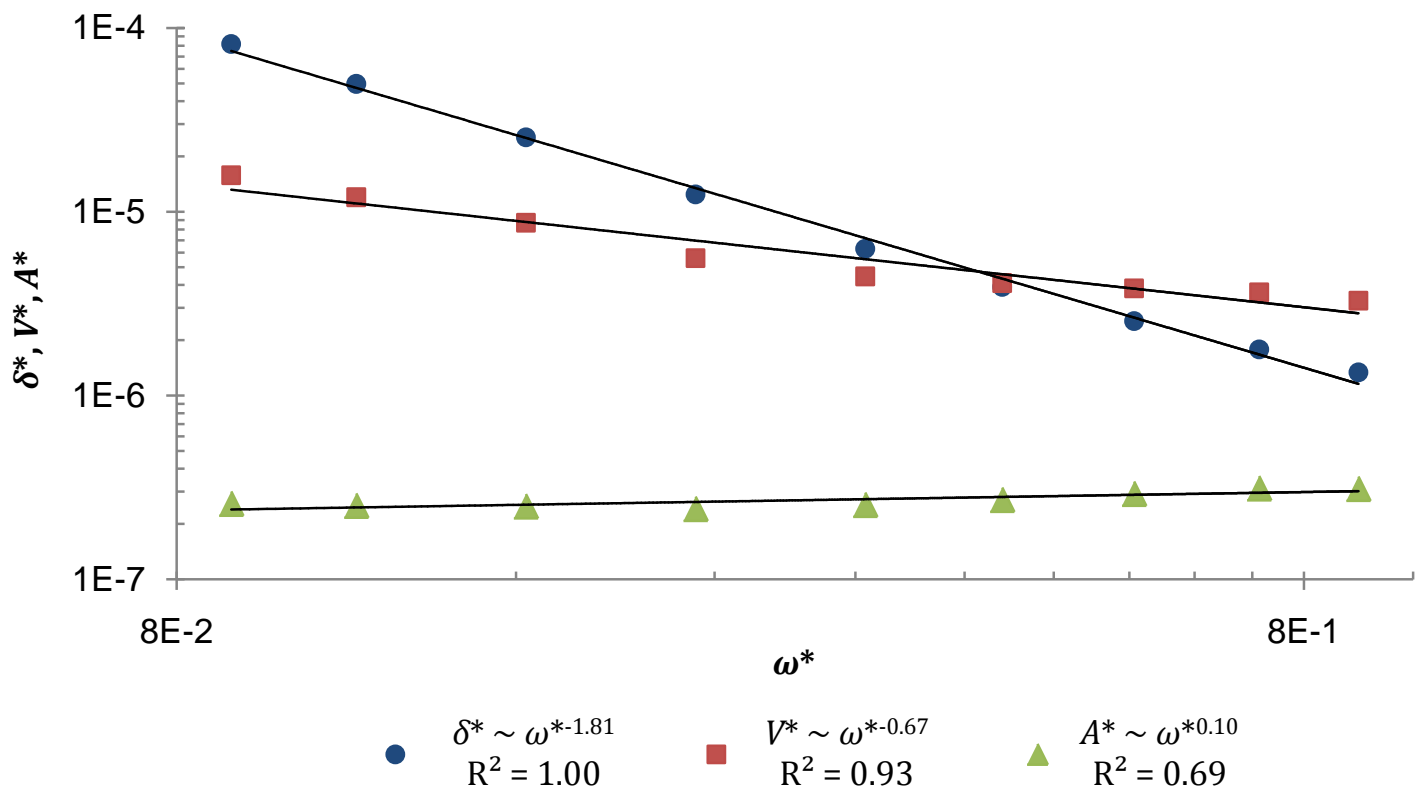

Figure 6-14 $\delta^{*}, V^{*}$, and $A^{*}$ as functions of $\omega^{*}$ for the long pipe model with $\operatorname{Re}_{\mathrm{D}}=$ $1.14 \times 10^{6}, t^{*}=0.0296, L^{*}=23.6, \rho^{*}=12.0$, and $\zeta=0.047$ 
The final first order influence of an independent variable examined was that of the damping ratio, $\zeta$. This was done with $R e_{D}=1.14 \times 10^{6}, t^{*}=0.0197, L^{*}=23.6, \rho^{*}=3.01$, and $\omega^{*}=0.473$. The results indicate a slight inverse relationship for all of the dependent variables as illustrated in Figure 6-15. The data indicates that $\delta^{*}$ is proportional to $\zeta^{-0.22}, V^{*}$ is proportional to $\zeta^{-0.51}$, and $A^{*}$ is proportional to $\zeta^{-0.35}$. Power law curves fit the data very well with average relative error values of $2.8 \%$ for $\delta^{*}$, 3.6\% for $V^{*}$, and 4.6\% for $A^{*}$.

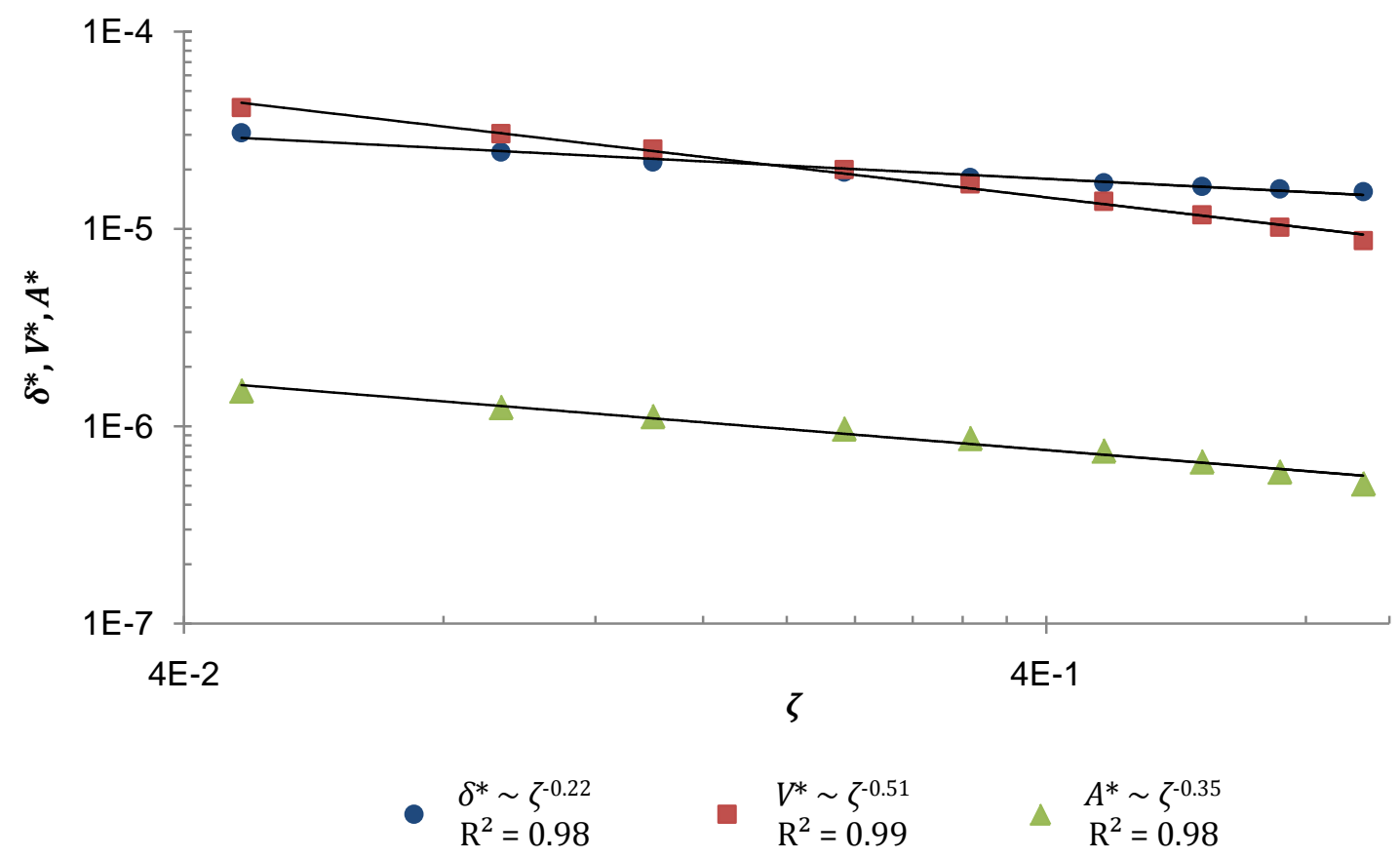

Figure 6-15 $\delta^{*}, V^{*}$, and $A^{*}$ as functions of $\zeta$ for the long pipe model with $R e_{\mathrm{D}}=$ $1.14 \times 10^{6}, t^{*}=0.0197, L^{*}=23.6, \rho^{*}=3.01$, and $\omega^{*}=0.473$

Because each of the variables indicates a power law relation within the range examined, the first order effects of each of the independent variables can be described by 
simply looking at the exponents for each of the power law curves fit to the data sets. The values of these exponents are given in Table 6-3.

Table 6-3 Summary of first order effects of all variables

\begin{tabular}{|c|c|c|c|c|c|c|}
\hline Variable & \multicolumn{3}{|c|}{ Short Pipe Power Fit Exponents } & \multicolumn{2}{c|}{ Long Pipe Power Fit Exponents } \\
\hline & $\delta^{*}$ & $\mathrm{~V}^{*}$ & $\mathrm{~A}^{*}$ & $\delta^{*}$ & $\mathrm{~V}^{*}$ & $\mathrm{~A}^{*}$ \\
\hline$R e_{D}$ & -0.26 & -0.30 & -0.38 & -0.20 & -0.12 & -0.18 \\
\hline$t^{*}$ & -2.05 & -1.96 & -0.90 & -1.16 & -1.06 & +0.04 \\
\hline$L^{*}$ & - & - & - & -0.72 & -0.34 & -0.16 \\
\hline$\rho^{*}$ & - & - & - & -1.00 & -1.00 & -1.00 \\
\hline$E^{*}$ or $\omega^{*}$ & -1.00 & -1.00 & -1.00 & -1.81 & -0.67 & +0.10 \\
\hline$\zeta$ & - & - & - & -0.22 & -0.51 & -0.35 \\
\hline
\end{tabular}

\subsection{Complete Functional Relationships}

All of the first order effects can be combined to generate a complete functional relationship for each of the dependant variables. All of the dependencies have been fit using power relationships of the form $D^{*} \propto A_{i}^{n_{i}}$, where $A_{i}$ is the $i$ th independent variable and $n_{i}$ is the $i$ th power-fit exponent. Multiplying each of the independent variables raised to the appropriate power results in a complete predictor of the dependant variable in terms of all independent variables. The results of all of the simulations are compared to the complete functional relationship and plotted. Each set of data is expected to be fit by a straight line through the origin. A linear fit through the origin has an $\mathrm{R}^{2}$ value of at least 0.98 for all of the dependant variables for both the long and short pipe. A plot of $\delta^{*}$ 
versus its complete functional relationship including all independent variables for the short pipe model is shown in Figure 6-16 along with a linear fit line having average relative error of $14 \%$. A plot of $V^{*}$ versus its complete functional relationship including all independent variables for the short pipe model is shown in Figure 6-17 along with a linear fit line having average relative error of $11 \%$. A plot of $A^{*}$ versus its complete functional relationship including all independent variables for the short pipe model is shown in Figure 6-18 along with a linear fit line having average relative error of 21\%. A plot of $\delta^{*}$ versus its complete functional relationship including all independent variables for the long pipe model is shown in Figure 6-19 along with a linear fit line having average relative error of $7.6 \%$. A plot of $V^{*}$ versus its complete functional relationship including all independent variables for the long pipe model is shown in Figure 6-20 along with a linear fit line having average relative error of 6.3\%. A plot of $A^{*}$ versus its complete functional relationship including all independent variables for the long pipe model is shown in Figure 6-21 along with a linear fit line having average relative error of 6.3\%. All of the full data sets are fit very well by straight lines intercepting the origin as expected.

The full functional relationships represented on the x-axis of each of these plots constitute the most important contribution of this research. These relationships describe the first-order contribution of each of the variables explored. Higher order interactions between variables are not represented in the functional relationships presented here. The functional relationships can be redimensionalized to allow one to pick out the specific contribution of each dimensional independent variable explored, but this exercise is not performed here. 


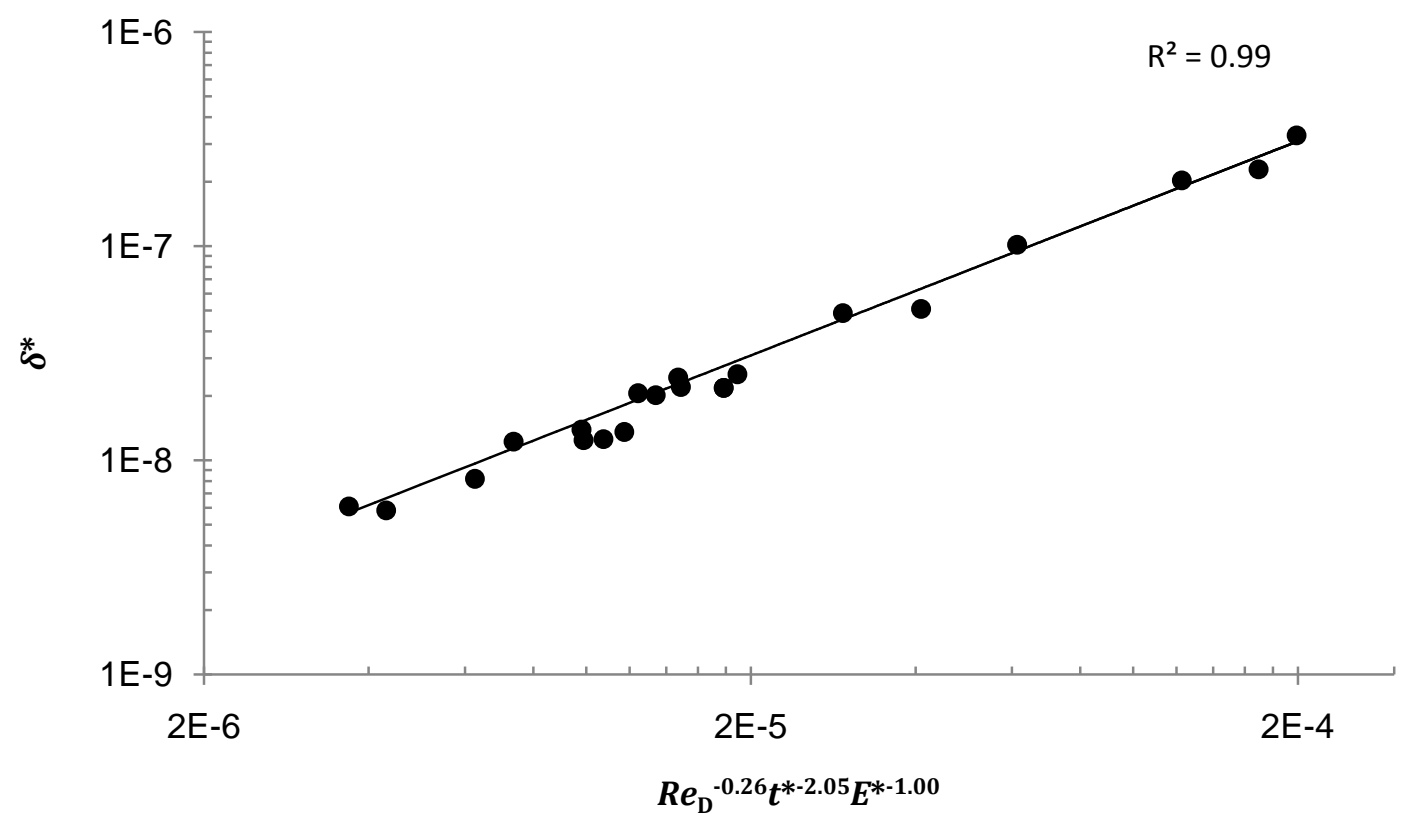

Figure 6-16 $\delta^{*}$ as a function of $\operatorname{Re}_{\mathrm{D}}{ }^{-0.26} t^{*-2.05} E^{*-1.00}$ for all short pipe model results

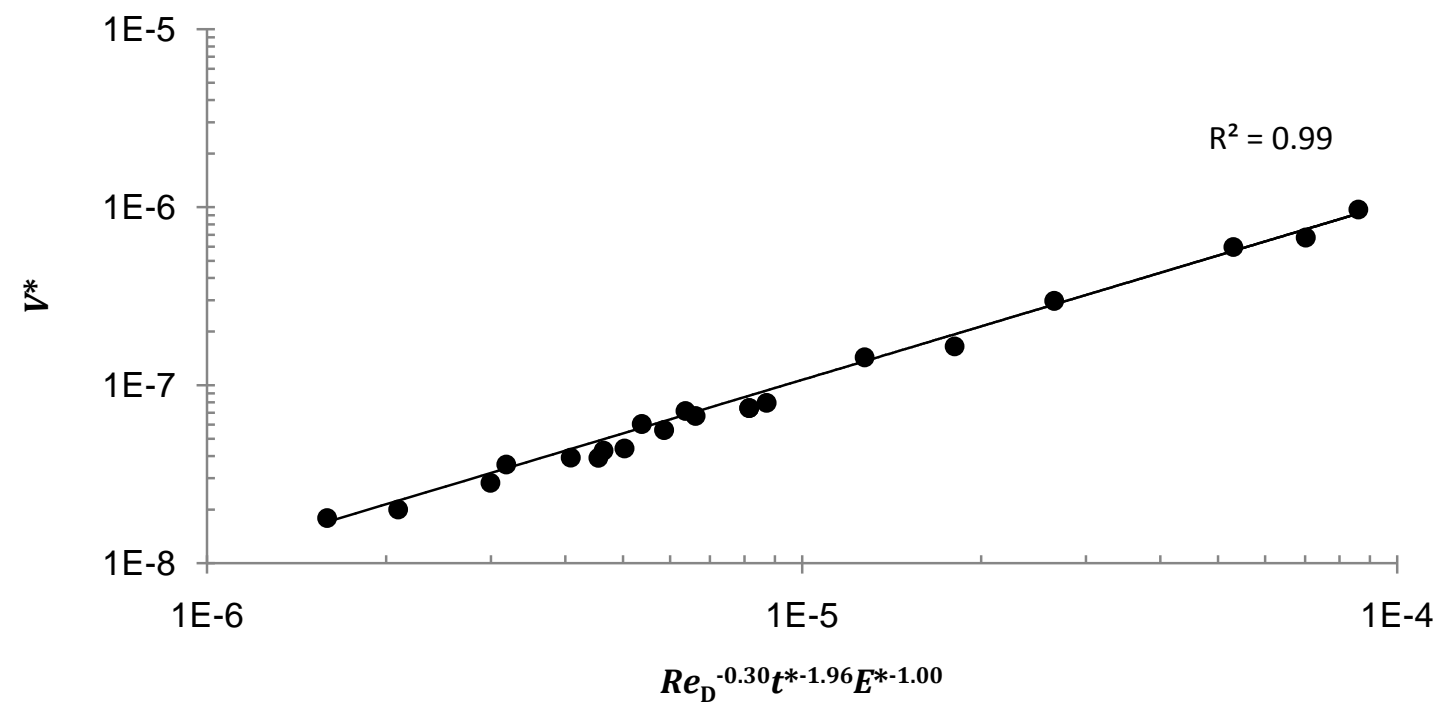

Figure 6-17 $V^{*}$ as a function of $R e_{\mathrm{D}}{ }^{-0.30} t^{*-1.96} E^{*-1.00}$ for all short pipe model results 


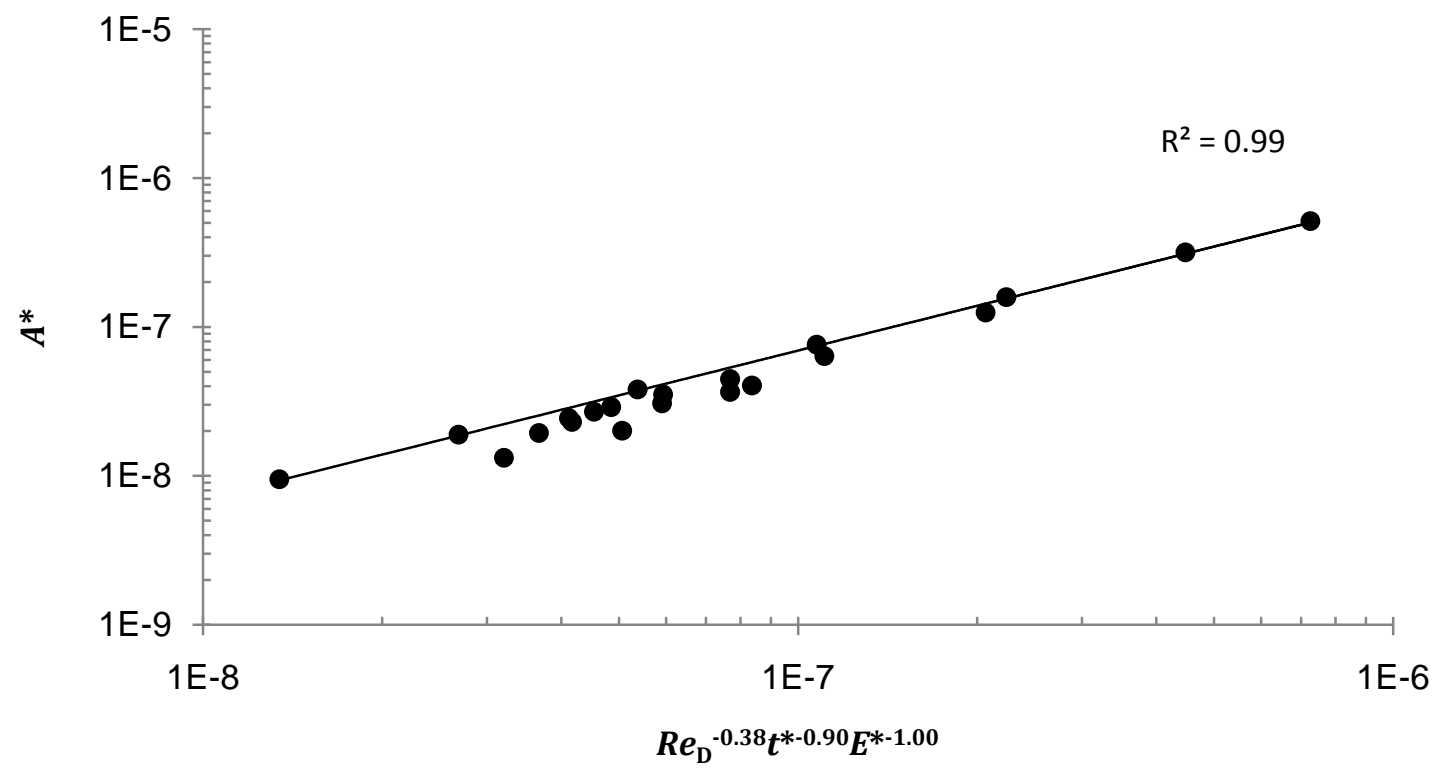

Figure 6-18 $A^{*}$ as a function of $\operatorname{Re}_{\mathrm{D}}^{-0.38} t^{*-0.90} E^{*-1.00}$ for all short pipe model results

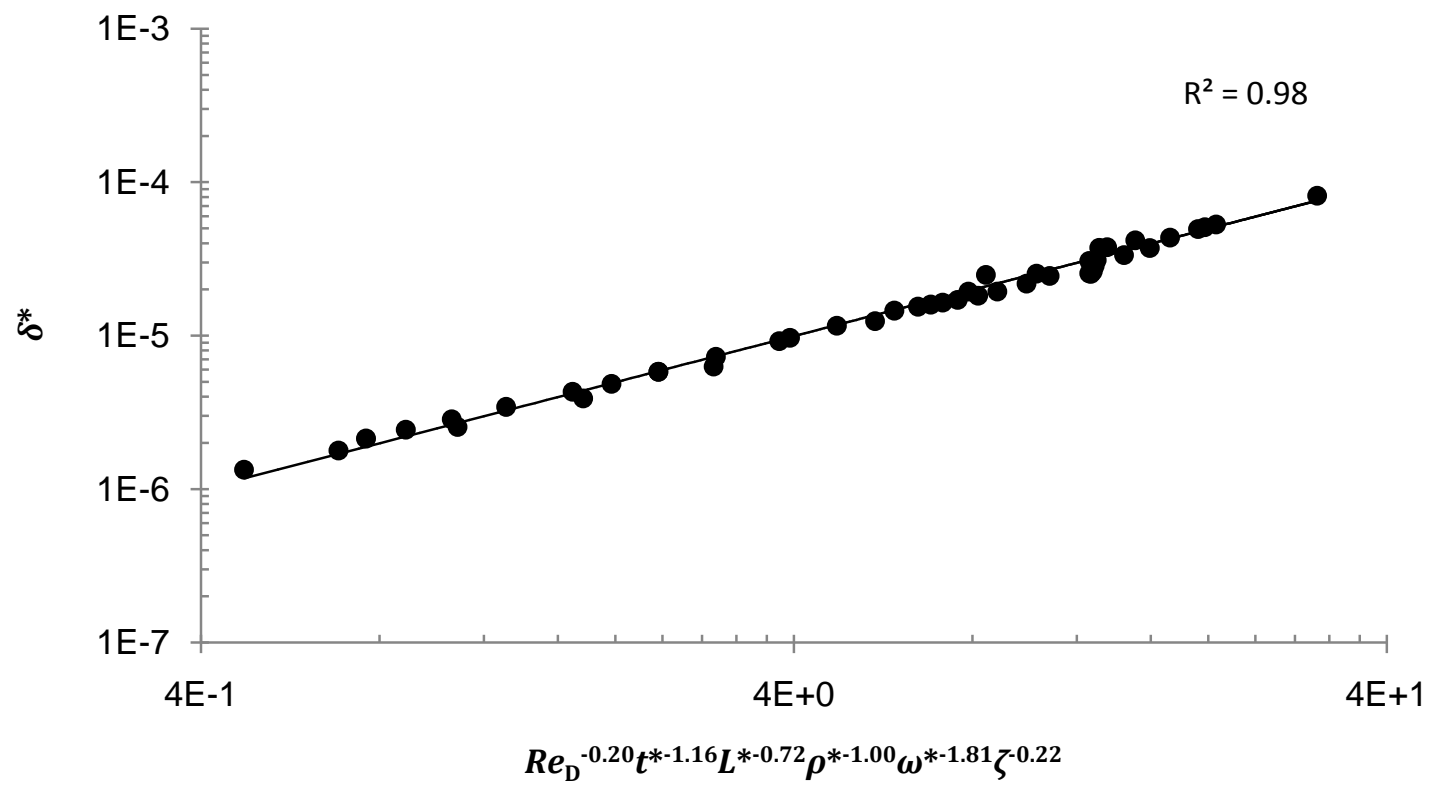

Figure 6-19 $\delta^{*}$ as a function of $R e_{\mathrm{D}}{ }^{-0.20} t^{*-1.16} L^{*-0.72} \rho^{*-1.00} \omega^{*-1.81} \zeta^{0.22}$ for all long pipe model results 


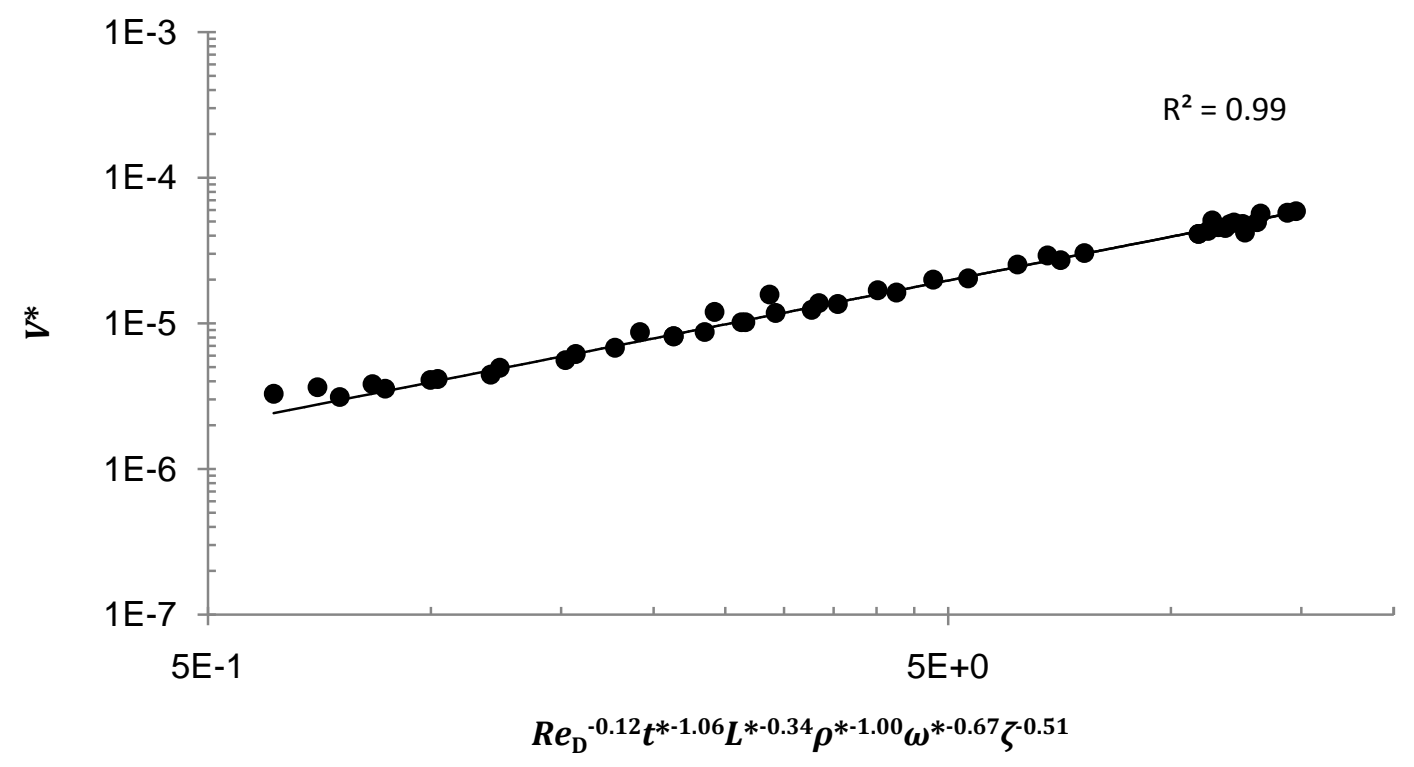

Figure 6-20 $V^{*}$ as a function of $R e_{\mathrm{D}}^{-0.12} t^{*-1.06} L^{*-0.34} \rho^{*-1.00} \omega^{*-0.67} \zeta^{0.51}$ for all long pipe model results

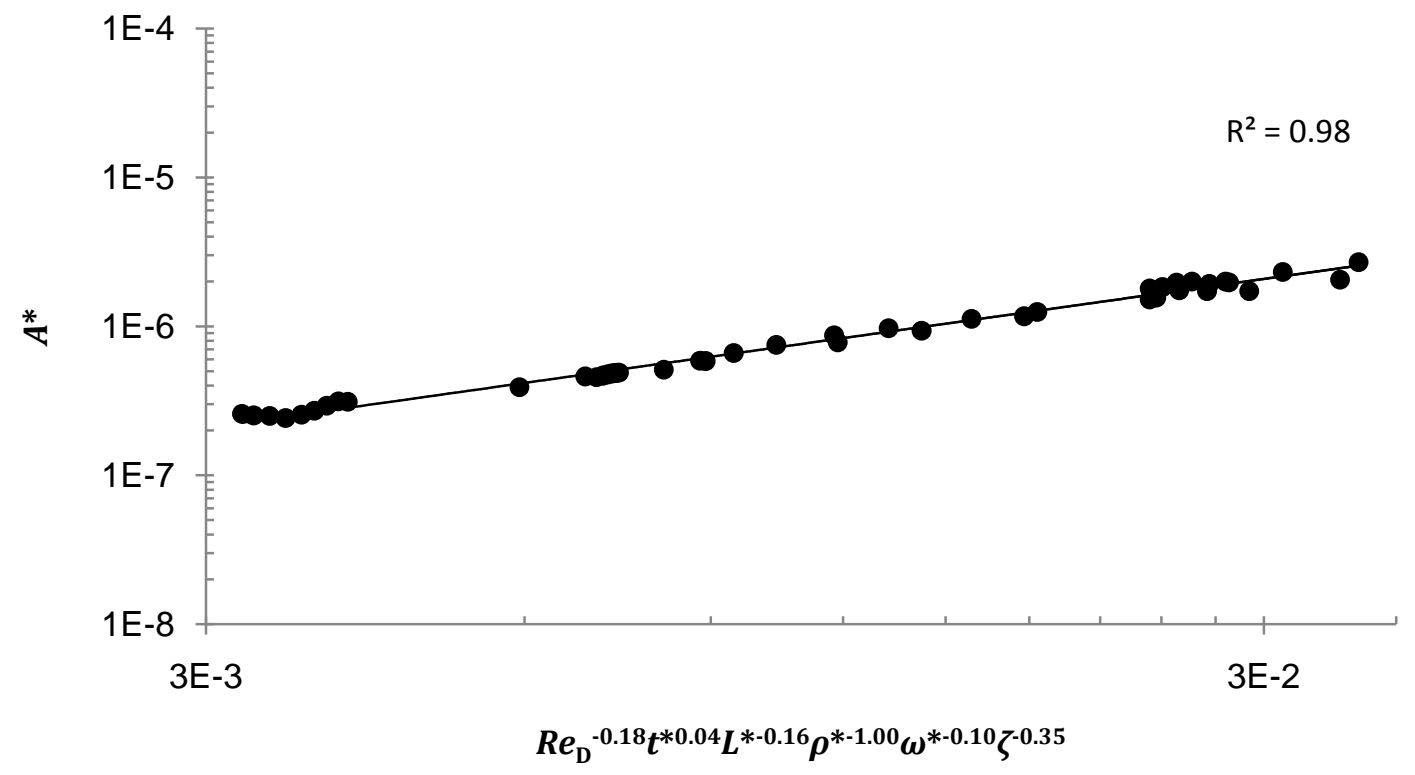

Figure 6-21 $A^{*}$ as a function of $R e_{\mathrm{D}}^{-0.18} t^{* 0.04} L^{*-0.16} \rho^{*-1.00} \omega^{*-0.10} \zeta^{0.35}$ for all long pipe model results 


\subsection{Comparison to Experiments}

There is very little experimental data covering the wide range of variables explored in this research. One of the primary goals of the numerical simulations was to cover a range of variables difficult to explore experimentally. There have, however, been some experimental efforts to characterize turbulent pipe flow induced vibration. Experimental data has primarily explored the effects of varying flow velocity. Additionally, the effects of pipe material and thickness have been explored, but only for 2 or 3 different values. Experimental results obtained by Evans predict that pipe wall acceleration is proportional to approximately $U^{2}$ [12]. Re-dimensionalizing the long pipe results presented in the previous section of this chapter results in a fluid velocity dependence of $A^{\prime} \propto U^{1.92}$. This is very close to the value predicted by experiment. It should also be noted that all experiments have used pipes with some degree of surface roughness. The effect of surface roughness is to reduce Reynolds number dependence of the wall shear stress. If the wall pressure fluctuations scale with shear stress as indicated by Figure 6-1, then the forcing function and pipe wall response would be expected to have less dependence on Reynolds number. Removing the Reynolds number dependence (which is small anyway) from the relationships predicted from this research results in a fluid velocity dependence exponent of 2.1 which is in the range of values (1.94 to 2.19) determined by Thompson [30].

Evans explored the use of 3 pipe materials over a range of flow rates from 7,000 to 23,000 g/s with water as the working fluid. The experimental pipes were all 3 inch (0.0762m) nominal diameter schedule 40 pipe made of PVC, aluminum, and stainless

steel. Water was used as the working fluid, and the pipe wall acceleration was measured 
using an accelerometer. Evans indicates that the experimental data from the PVC pipe is fit very well by the relation $A^{\prime}=2.98 \mathrm{e}-11 \times \mathrm{Q}^{2}$, where $\mathrm{Q}$ is the flow rate in grams per second. The aluminum pipe data is fit by the same curve divided by a constant 1.2, and the steel pipe data is fit by the same curve as the PVC divided by a constant 2.2. Evans proposes multiplying the data by $\sqrt{\rho_{P} / \rho_{f}}$, where $\rho_{P}$ is the pipe material density and $\rho_{f}$ is the fluid density, to collapse all three sets onto a single curve [12]. The present numerical simulations suggest that multiplying the data instead by $\rho^{*}$ will also cause the data to collapse since $A^{*} \propto 1 / \rho^{*}$. Figure 6-22 shows a plot comparing the method used by Evans with the curve fit generated using the functional relationships from this research. Both methods allow the data to be fit closely by a single curve, although the fit is slightly better with $\mathrm{R}^{2}=0.998$ for the method using $\rho^{*}$, compared to $\mathrm{R}^{2}=0.993$ for the method using $\sqrt{\rho_{P} / \rho_{f}}$.

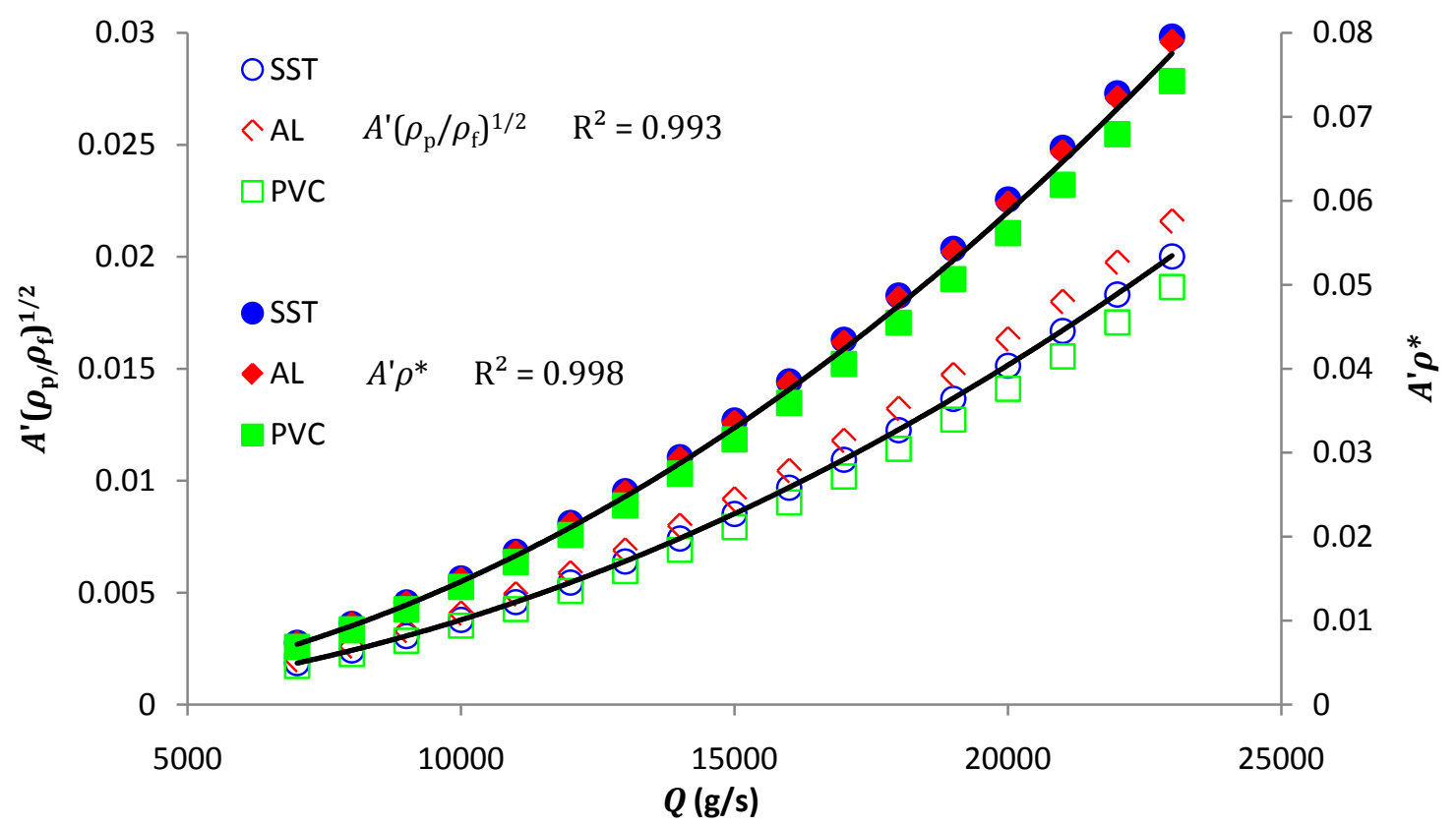

Figure 6-22 Comparison to Evans experimental data 
Thompson used only a single pipe material (PVC) for all experiments, but used both schedule 40 and schedule 80 pipe to determine the effects of varying thickness [30]. The pipe wall acceleration was determined using an accelerometer and the fluid velocity was varied by controlling the speed of the driving pump. The PVC test sections were isolated from the pump vibrations by sections of rubber pipe upstream and downstream of the test section. Six sets of data are used here to check the numerical simulation, each corresponding to a different pipe test section. Table 6-4 shows the geometry of each section used in the experiments performed by Thompson. Water was the working fluid used for all of the experiments.

Table 6-4 Thompson experiment pipe geometry

\begin{tabular}{|c|c|c|c|}
\hline Nominal Pipe Size & Pipe Schedule & Inner Diameter, $D$ & Wall Thickness, $t$ \\
\hline 4 inch & 40 & $0.1023 \mathrm{~m}$ & $6.02 \mathrm{~mm}$ \\
\hline 4 inch & 80 & $0.0972 \mathrm{~m}$ & $8.56 \mathrm{~mm}$ \\
\hline 3 inch & 40 & $0.0779 \mathrm{~m}$ & $5.49 \mathrm{~mm}$ \\
\hline 3 inch & 80 & $0.0737 \mathrm{~m}$ & $7.62 \mathrm{~mm}$ \\
\hline 2 inch & 40 & $0.0525 \mathrm{~m}$ & $3.91 \mathrm{~mm}$ \\
\hline 2 inch & 80 & $0.0493 \mathrm{~m}$ & $5.54 \mathrm{~mm}$ \\
\hline
\end{tabular}

The numerical simulations suggest that $A^{\prime}$ should be nearly inversely proportional to $t^{*}$ because $A^{*} \propto t^{* 0.04}$. Including the effects of varying $t^{*}$ and varying $\rho^{*}$ (which also changes with thickness) while neglecting the influence of Reynolds number, results in the prediction that $A^{\prime} \propto U^{2.1} /\left(\rho^{*} t^{* 0.96}\right)$. A plot of the standard deviation of the pipe wall acceleration verses this prediction expression is shown in Figure 6-23. The data for each 
nominal pipe size is fit with a line passing through the origin resulting in a coefficient of determination of $\mathrm{R}^{2}=0.95$ for the 4 inch pipe data, $\mathrm{R}^{2}=0.98$ for the 3 inch data, and $\mathrm{R}^{2}$ $=0.93$ for the 2 inch data. Each pair of data sets (schedule 40 and schedule 80) collapses to a straight line when accounting for changes in pipe wall thickness and average fluid velocity.

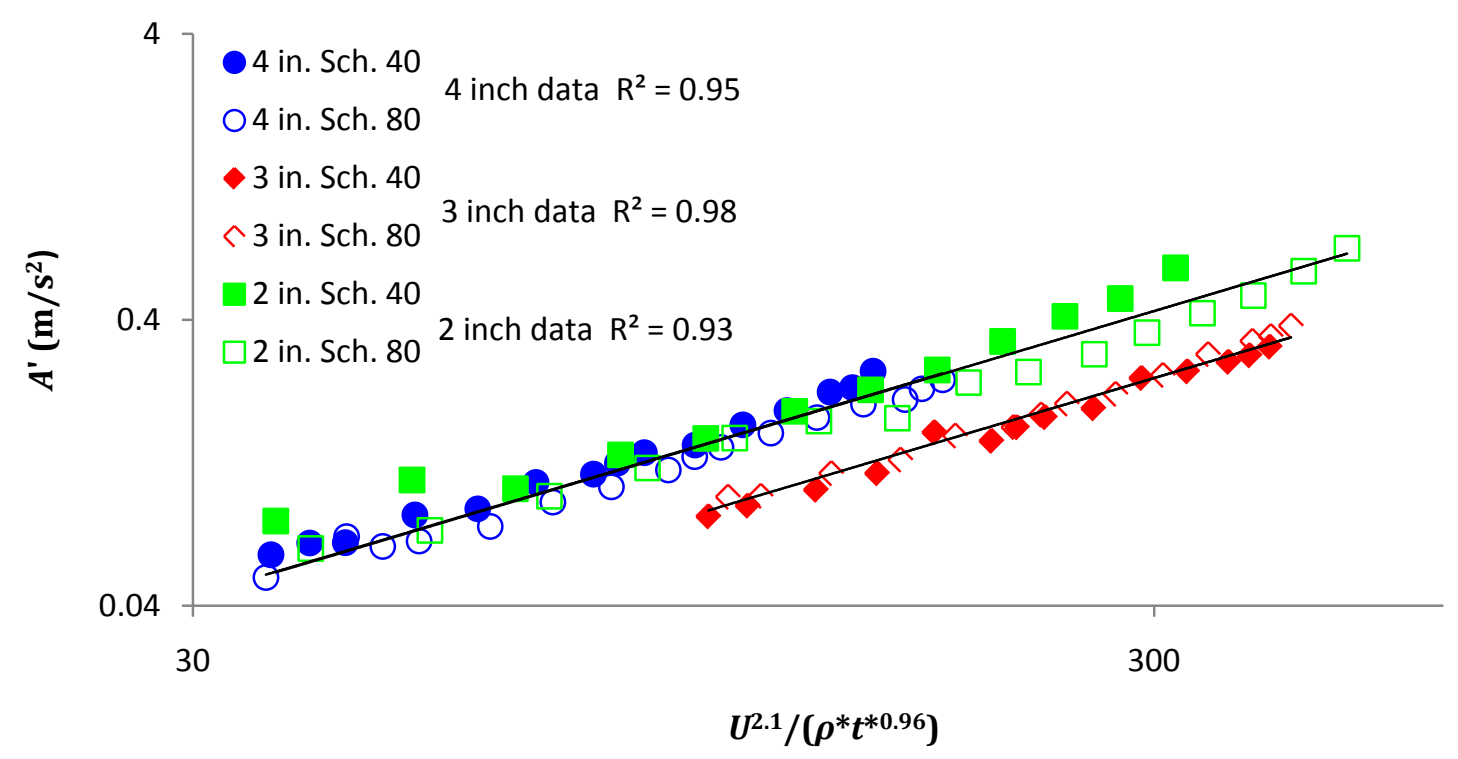

Figure 6-23 Comparison to Thompson experimental data with linear fit

The numerical model relations fit the available experimental data, although it is difficult to account for differences in pipe support boundary conditions, effective pipe length and structural damping in an experiment. The inherent strength of the numerical model is the ability to control these additional parameters independently. Accounting only for first order effects of the parameters varied for the numerical simulations, results in predictions that are able to match experimental data for the standard deviation of pipe wall acceleration. 


\section{Conclusion}

\subsection{Summary}

A numerical model of fully developed turbulent pipe flow based on LES has been developed and solved for 8 different Reynolds numbers. The LES model has been verified and validated to ensure accuracy of the solutions obtained through its implementation. The solutions from this fluid model have been used to approximate the fluctuating pressure field on the inside surface of a pipe. This fluctuating pressure field has then been applied as external loads on the surface of a structural model of a segment of pipe. The structural model using FEA has also been verified and validated. The magnitude of the pipe wall motion due to the applied pressure fields has been explored for a range of pipe geometric and material property variables. A complete set of nondimensional parameters that represents all of the variables explored has been used to generalize the results.

The results indicate that there is a fundamental difference in the response of a short pipe compared to the response of a long pipe. The short pipe responds immediately to changes in the pressure field and does not require the pipe inertia to be considered. The short pipe tends to respond to local pressure variations and is more sensitive to changes in wall thickness than a long pipe. The short pipe model indicates reduced response to 
higher frequencies in the pressure fluctuations which correspond to smaller turbulence length scales. The long pipe model is influenced by the pipe inertia and must include transient structural effects. The long pipe tends to respond primarily by bending like a beam. The long pipe also shows decreased response to the highest frequency small-scale pressure fluctuations, but also indicates heightened response at middle frequencies that correspond to the natural frequencies of the pipe.

A functional relationship between 3 pipe motion dependant variables and 6 dimensionless independent variables exerting influence has been developed by exploring first order effects of each variable. The results can be summarized by these functional relationships as shown in Table 7-1. These results were compared to available experimental data, and indicate that the numerical model used here has produced results with behavior similar to experiments.

Table 7-1 Functional relationships: Result summary

\begin{tabular}{|c|}
\hline Short Pipe \\
\hline$\delta^{*} \propto R e_{D}^{-0.26} t^{*-2.05} E^{*-1.0}$ \\
\hline$V^{*} \propto R e_{D}^{-0.30} t^{*-1.96} E^{*-1.0}$ \\
\hline$A^{*} \propto R e_{D}^{-0.38} t^{*-0.90} E^{*-1.0}$ \\
\hline Long Pipe \\
\hline$\delta^{*} \propto R e_{D}^{-0.20} t^{*-1.16} L^{*-0.72} \rho^{*-1.0} \omega^{*-1.81} \zeta^{-0.22}$ \\
\hline$V^{*} \propto R e_{D}^{-0.12} t^{*-1.06} L^{*-0.34} \rho^{*-1.0} \omega^{*-0.67} \zeta^{-0.51}$ \\
\hline$A^{*} \propto R e_{D}^{-0.18} t^{*+0.04} L^{*-0.16} \rho^{*-1.0} \omega^{*+0.10} \zeta^{-0.35}$ \\
\hline
\end{tabular}




\subsection{Limitations}

The LES model used here is still limited by available computational resources. The velocity profile of the highest Reynolds number flow explored is unable to be resolved fully near the wall. The use of wall modeling adds additional approximations that result in somewhat inaccurate velocity profiles in this region. The LES fluid domain is also short compared to many of the structural domains, requiring the pressure field to be repeated in the axial direction. Any pressure imbalance in the field results in a net force that causes the pipe to bend. This net force is effectively multiplied when the pressure field is repeated which may lead to larger bending motions than would exist for a model using a full-length fluid domain. The solutions have been limited to 1000 time steps to reduce the computation time required for the fluid model. While spatially averaged quantities become statistically steady within 1000 time steps, the local values of the wall shear stress and the standard deviation of pressure fluctuations are not statistically steady at 1000 time steps. A longer solution (more time steps) would produce results with less noise.

\subsection{Recommendations}

In this thesis the first order effects of the variables of interest were explored. It is recommended that additional research using this approach consider higher order effects by performing a more complete set of simulations that could be used to determine interaction between independent variables. Future research could also use a longer fluid domain or more time steps to achieve more accurate results as additional computation power becomes available. Combined efforts including experimental work could also be 
used to develop an improved set of boundary conditions for the structural model, or a way to specify boundary conditions to model a particular experimental set-up for direct comparison of results. The effect of pipe length has already been shown through this research to be heavily involved in complex effects, and could be the focus of future research using this type of model. 


\section{References}

1. Eisinger, F. L. "Desinging Piping Systems Against Acoustically Induced Structural Fatigue." Journal of Pressure Vessel Technology 119 (1997): 379-383.

2. Evans, R., Wilkins, C., Goodrich, L., Blotter, J. Method and apparatus for measuring the mass flow rate of a fluid. United States Patent 6,412,352. July 2, 2002.

3. Weaver, D. S., Zaida, S., Au-Yang, M. K., Chen S. S., Paidoussis, M.P., and Pettigrew, M.J. "Flow-Induced Vibrations in Power and Process Plant Components - Progress and Prospects." Journal of Pressure Vessel Technology 122 (August 2000): 339-348.

4. Durant, C., Robert, G., Filippi, P., Mattei, P. "Vibroacoustic Response of a Thin Cylindrical Shell Excited by a Turbulent Internal Flow: Comparison Between Numerical Prediction and Experimentation." Journal of Sound and Vibration 229 (2000): 1115-1155.

5. Vassilev, V. M., and Djonderjorov, P. A. "Dynamic Stability of Viscoelastic Pipes on Elastic Foundations of Variable Modulus." Journal of Sound and Vibration 297 (2006): 414-419.

6. Paidoussis, M. P. "The Canonical Problem of the Fluid-Conveying Pipe and Radiation of the Knowledge Gained to Other Dynamics Problems Across Applied Mechanics." Journal of Sound and Vibration 310 (2008): 462-492.

7. Evans, R. P., Blotter, J.D., and Stephens, A.G. "Flow Rate Measurements Using Flow-Induced Pipe Vibration." Journal of Fluids Engineering 126 (March 2004): 280-285.

8. Weyers, P. The Vibration and Acoustic Radiation of Thin-Walled Cylinders Caused by Internal Turbulent Flow. PhD Thesis, Pasadena, California: California Institute of Technology, 1959.

9. Birgersson, F., Finnveden, S., and Robert, G. "Modelling Turbulence-Induced Vibration of Pipes With a Spectral Finite Element Method." Journal of sound and Vibration 278 (2004): 749-772. 
10. Bochkarev, S. A., and Matveenko, V. P. "Numerical Study of the Influence of Boundary Conditions on the Dynamic Behavior of a Cylindrical Shell Conveying a Fluid." Mechanics of Solids 43 (2008): 477-486.

11. Pittard, M. T. Large Eddy Simulation Based Turbulent Flow-Induced Vibration of Fully Developed Pipe Flow. MS Thesis, Provo, Utah: Brigham Young University, 2003.

12. Evans, R. P. Mass Flow Measurement Through Flow-Iduced Pipe Vibration. PhD Dissertation, Pocatello, Idaho: Idaho State University, 2004.

13. Kim, B. S. "Analysis of Fluid Induced Vibration of Cryogenic Pipes in Consideration of the Cooling Effect." Journal of Mechanical Science and Technology 22 (2008): 2375-2385.

14. Xu, J., and Yang, Q. "Flow-Induced Internal Resonances and Mode Exchange in Horizontal Cantilevered Pipe Conveying Fluid (I)." Applied Mathematics and Mechanics (English Edition) 27 (2006): 935-941.

15. Saito, N., Miyano, H., and Furukawa, S. "Study on Vibration Response of Pipes Induced by Internal FLow." Pressure Vessels and Piping Conference. Nashville, Tennessee: ASME Pressure Vessels Piping Div Publ PVP, 1990. 233-238.

16. Durant, C. and Robert, G. "Vibro-Acoustic Response of a Pipe Excited by a Turbulent Internal Flow." Flow Turbulence and Combustion 61 (1998): 55-69.

17. Seo, Y., Jeong, W., Jeoung, S., Oh, J., and Yoo, W. "Finite Element Analysis of Forced Vibration for a Pipe Conveying Harmonically Pulsating Fluid." JSME International Journal 48 (2005): 688-694.

18. Finnveden, S., Birgersson, F., Ross, U., and Kremer, T. "A Model of Wall Pressure Correlation for Prediction of Turbulence-Induced Vibration." Journal of Fluids and Structures 20 (2005): 1127-1143.

19. Ansys CFX V 11.0 Users Manual.

20. Smagorinsky, J. S. "General Circulation Experiments with the Primitive Equations." Monthly Weather 91 (1963): 99-164

21. Eggels, J. G. M. Direct and Large Eddy Simulation of Turbulent Flow in a Cylindrical Pipe Geometry. PhD Thesis, Delft, The Netherlands: Delft University Press, 1994. 
22. Rudman, M. and Blackburn, H. M. "Large Eddy Simulation of Turbulent Pipe Flow." Second International Conference of CFD in the Minerals and Process Industries CSIRO. Melbourne, Australia, 1999. 503-508.

23. Patanker, S. Numerical Heat Transfer and Fluid Flow. New York, NY: Hemisphere Pub. Corp., 1980

24. Piomelli, U. "Large-Eddy Simulation: Where We Stand." Advances in DNS/LES AFSOR. Ruston, Lousiana, 1997. 93-104.

25. Karmen, Th. V. Mechanical Similitude and Turbulence. Technical Memorandum No. 611, National Advisory Committee for Aeronautics, 1931.

26. Guo, J. and Julien, P.Y. "Modified Log-Wake Law for Turbulent Flow in Smooth Pipes." Journal of Hydraulic Research 41 (2003): 493-501.

27. Frarey, J.L. "Pitfalls in the Analysis of Machinery Vibration Measurements." Sound and Vibration, 2002: 18-24.

28. Pope, S. Turbulent Flows. New York, NY: Cambridge University Press, 2000.

29. Craig, R.R., and Kurdila, A.J. Fundamentals of Structural Dynamics. Hoboken, NJ: John Wiley \& Sons Inc., 2006.

30. Thompson, A. Experimental Characterization of Flow Induced Vibration in Fully Developed Turbulent Pipe Flow. MS Thesis, Provo, Utah: Brigham Young University, 2009. 


\section{Appendix A}

\section{Mesh Creation Instructions}

The mesh used for the fluid model was created using ANSYS ${ }^{\circledR}$ ICEM CFD $^{\mathrm{TM}}$. It is a utility intended strictly for generating meshes to be used for computational fluid dynamics (CFD) simulations. The meshing program can import geometry from a variety of computer aided drafting (CAD) packages, or can be used to create geometry from scratch. Because of the simple cylindrical geometry used here, the geometry is created directly in ICEM CFD ${ }^{\mathrm{TM}}$. O-grid type blocking is then created to allow a higher quality mesh near the pipe wall. Finally, the mesh parameters are set, and the mesh is generated and exported. The steps for generating the fluid mesh using ICEM CFD ${ }^{\mathrm{TM}}$ are described below.

\section{A.1 Geometry}

The fluid domain consists of a cylinder that is $0.3 \mathrm{~m}$ long and $0.1015 \mathrm{~m}$ in diameter. A Cartesian coordinate system is used, with the x-dimension corresponding to the axis of the cylinder. First a cylindrical surface is created using standard shapes or primitives (Geometry $\rightarrow$ Create/Modify Surface $\rightarrow$ Cylinder). The first vertex is created at the

point $\left[\begin{array}{lll}0 & 0 & 0\end{array}\right]$, and the second vertex is set to $\left[\begin{array}{lll}0.3 & 0 & 0\end{array}\right]$. The radius is chosen to be $\mathrm{D} / 2$ or $0.05075 \mathrm{~m}$. The circular edges at both the inlet and outlet are subdivided into four 
segments using Geometry $\rightarrow$ Create/Modify Curve $\rightarrow$ Segment Curve to aid in the blocking process. The cylindrical domain with subdivided edges is shown in Figure A-1. With the basic geometry in place the surfaces can be named and the fluid interior defined. Display Tree $\rightarrow$ Parts $\rightarrow$ Create Part is used, and the surface on the yz plane is selected and named "Inlet." The process is repeated by selecting the surface opposite the inlet and renaming it "Outlet.” Finally, the cylindrical surface is selected and renamed to "Wall.” Creating these parts will allow different boundary conditions to be defined for each one when it comes time to start a simulation. The fluid interior is created by using Geometry $\rightarrow$ Create Body. The part name is changed to "fluid interior” or some other suitable name. The Centroid of 2 points option is used, and the 2 screen locations arrow is selected. Any two points in the interior of the cylinder can be used, or a point on one side of the inlet and the opposite side of the outlet can be selected. This will define the solid region contained by all surfaces as the fluid region. With the new parts created the Geometry $\rightarrow$ Repair Geometry $\rightarrow$ Build Diagnostic Topology tool is used with all default settings to remove any unused surfaces and points. This step helps avoid any confusion when generating the blocking in the next steps. 


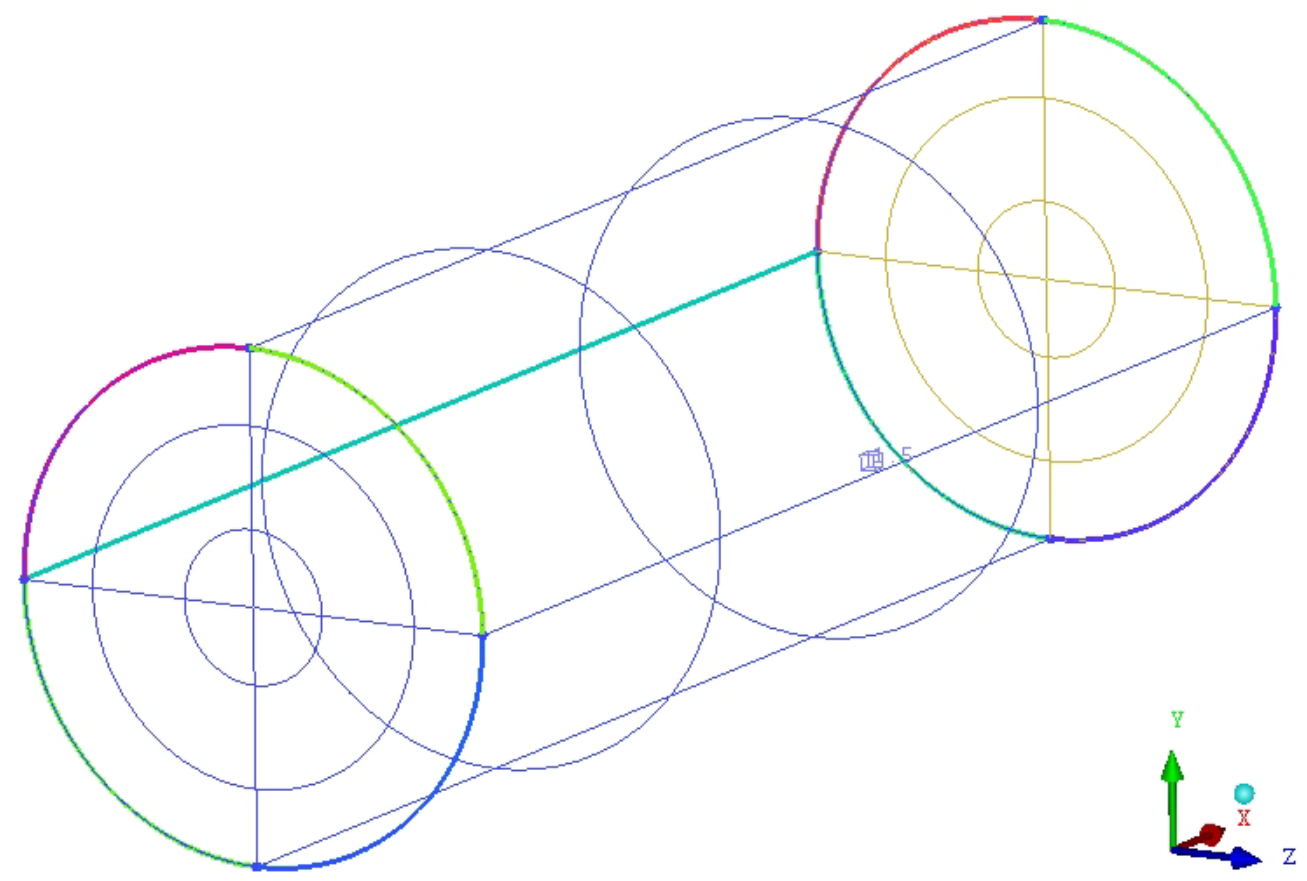

Figure A-1 Basic geometry showing subdivided curves

\section{A.2 Blocking}

Once the geometry is done, a block is created and linked to the geometry. This is done by creating a new block as a bounding box around the entire domain (Blocking $\rightarrow$ Create Block $\rightarrow$ 3D Bounding Box). Associations are then defined such that each vertex of the block is associated with a point between adjacent segments of the curves at the inlet and outlet (Blocking $\rightarrow$ Associate $\rightarrow$ Associate Vertex). This will result in the block becoming contained within the cylinder. The edges of the block are then each associated with their respective nearest curved segment (Blocking $\rightarrow$ Associate $\rightarrow$ Associate Edge to Curve). An image of the geometry with blocking is shown in Figure 
A-2 with green arrows indicating edge associations. The block is also resized by a factor of 1.7 in the $y$ and $\mathrm{z}$ directions using the Blocking $\rightarrow$ Transform Blocks $\rightarrow$ Scale Blocks function. At this point the block can be split using the Blocking $\rightarrow$ Split Blocks $\rightarrow$ Ogrid Block function to allow superior grid properties at the pipe wall. The block and the inlet and outlet surfaces must be selected for this operation to work correctly. After splitting there will be five separate blocks, with the four outside blocks allowing the grid to become perpendicular to the pipe wall while remaining uniform in the central region of the fluid defined by the inner rectangular block. The resized block with O-grid split is shown in Figure A-3.

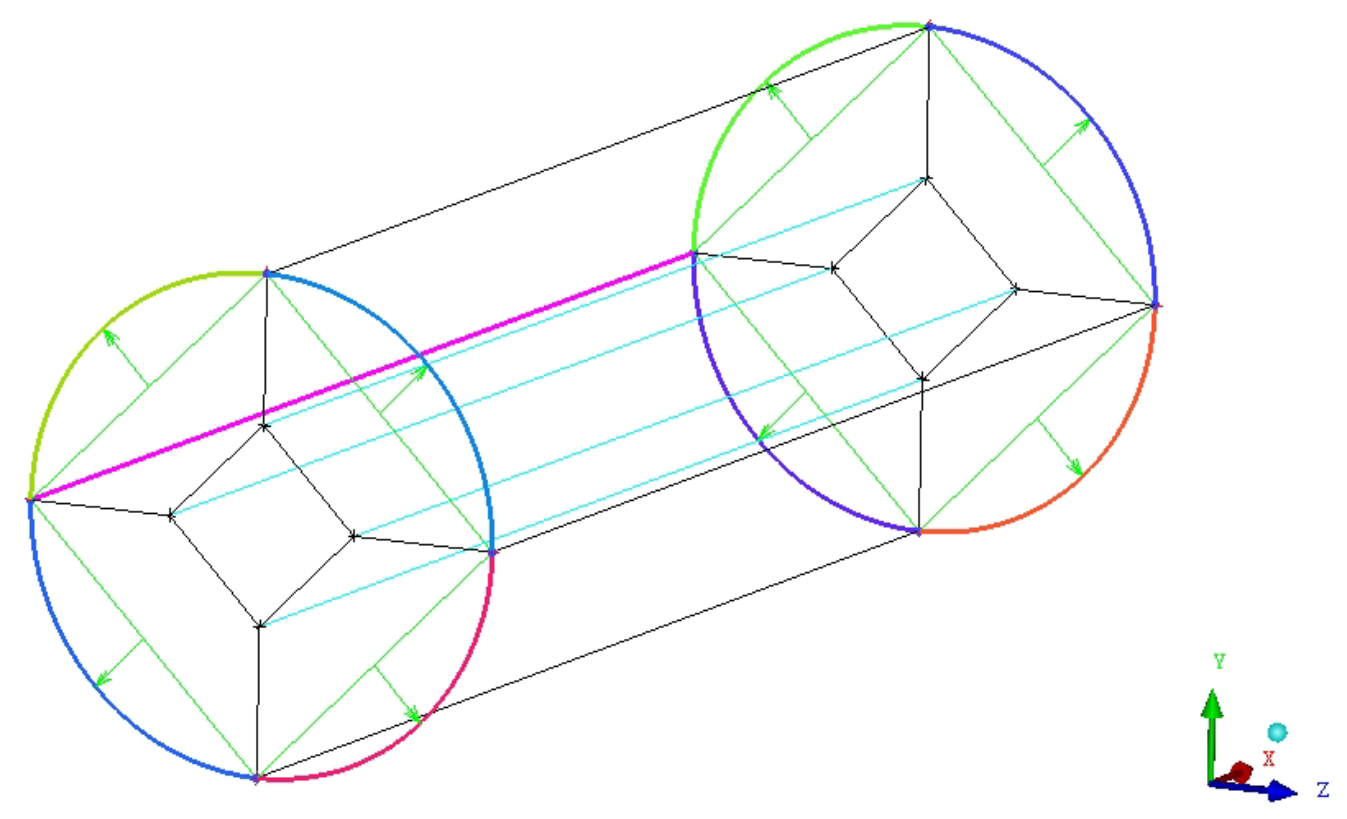

Figure A-2 Block with edges associated to geometry 


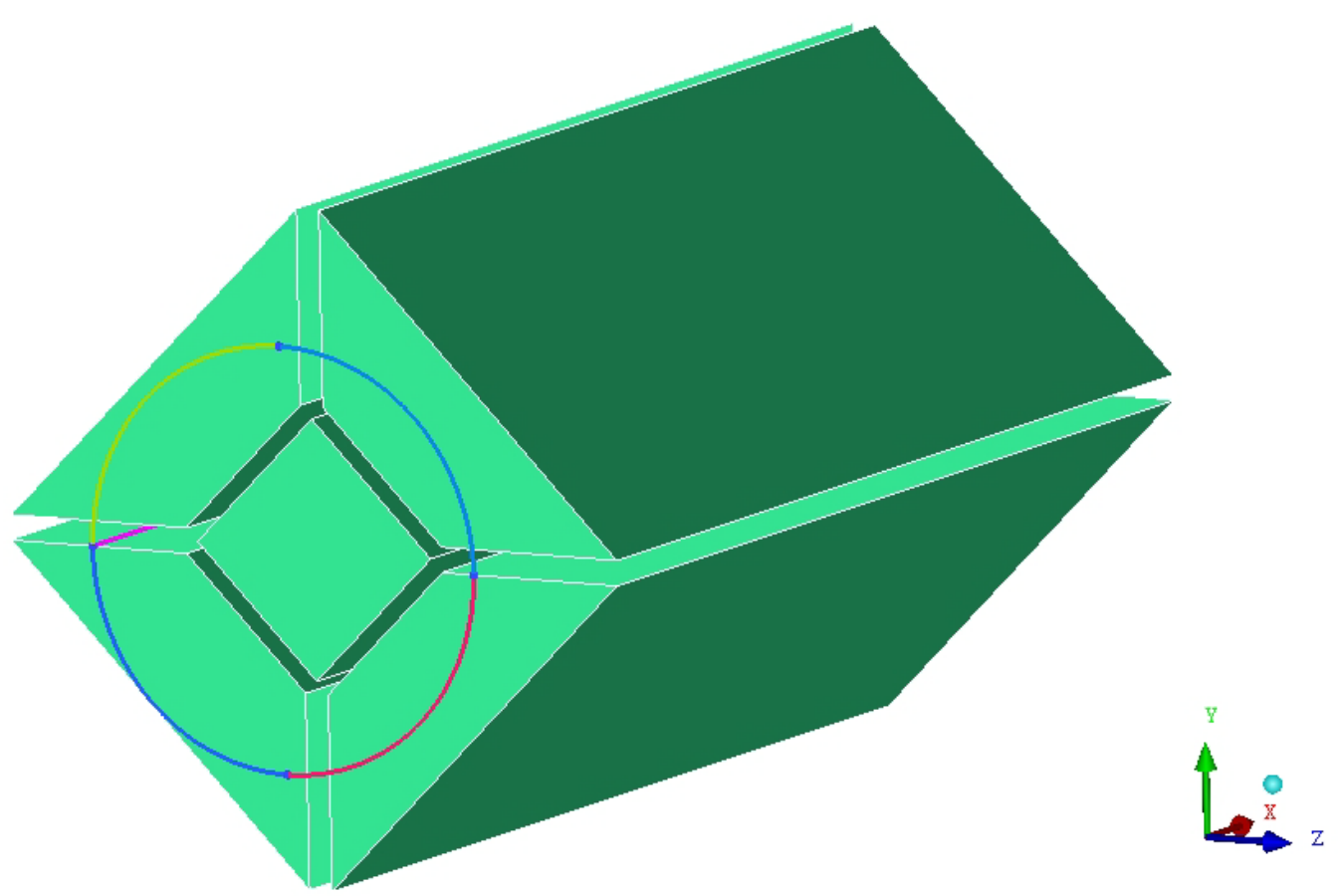

Figure A-3 Resized block split into sections using O-grid

\section{A.3 Mesh Parameters}

The Mesh $\rightarrow$ Surface Mesh Setup tool is used to apply arbitrary global mesh size parameters, but specific parameters are applied to selected edges under Blocking $\rightarrow$ Premesh Params $\rightarrow$ Edge Params. The four edges parallel to the $\mathrm{x}$-axis are selected and set to contain 180 nodes with Mesh Law set to uniform. The eight outside edges (four around the inlet and four around the outlet) are then selected and set to contain 60 nodes with Mesh Law still set to uniform. Finally, all of the radially oriented edges are selected and set to contain 30 nodes and Mesh Law set to From-Graphs. A distribution that results in a finer mesh near the pipe wall is used. The Pre-mesh is computed using Display Tree $\rightarrow$ Premesh $\rightarrow$ Recompute. An end view of the mesh is shown in Figure A-4. The mesh quality can be checked using the Pre-mesh Quality Histograms. 


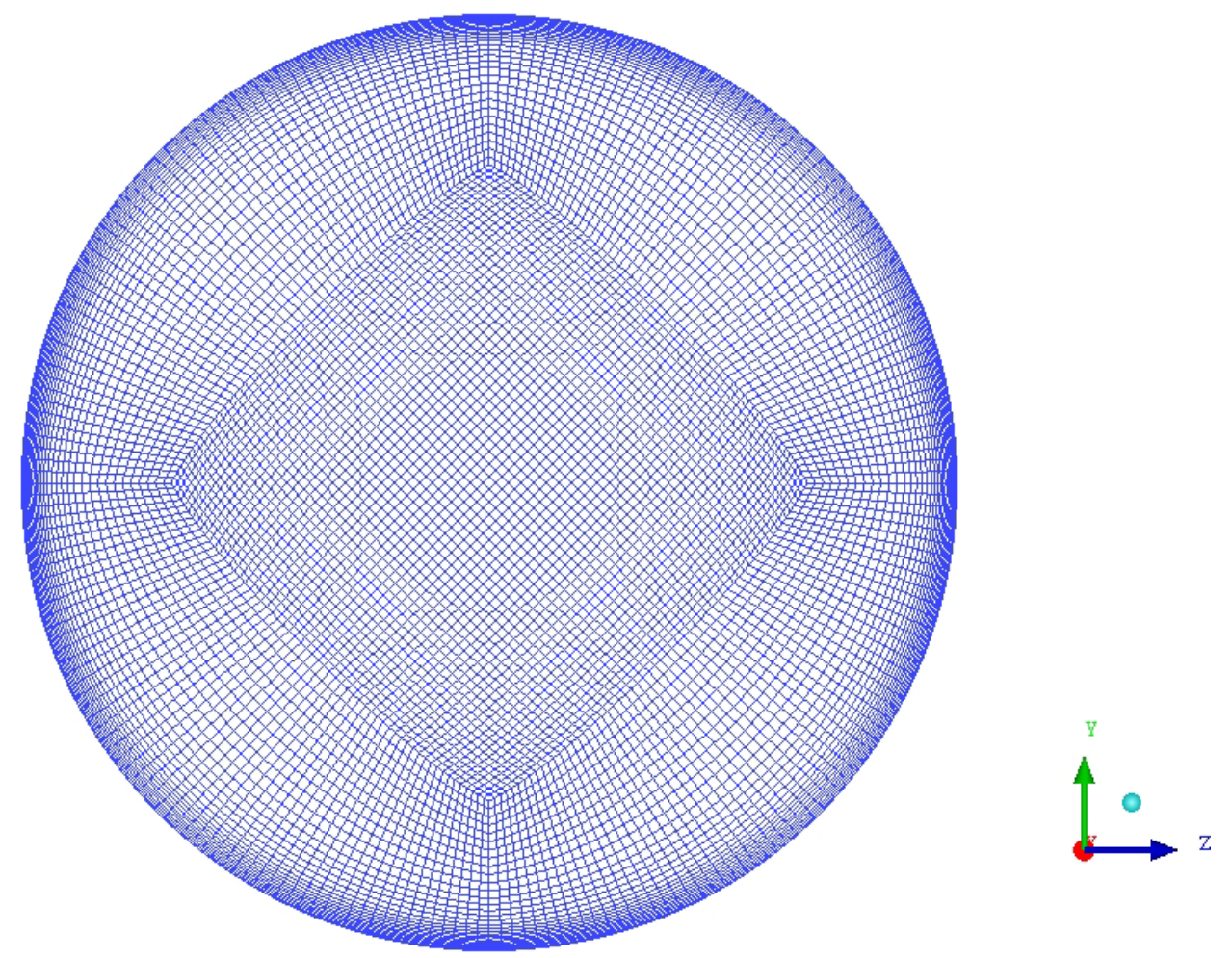

Figure A-4 Completed mesh as seen from the inlet end of the pipe

\section{A.4 Exporting Mesh for use in $\mathrm{CFX}^{\circledR}$}

After verifying mesh quality by looking at properties such as skewness, angles, and 2x2x2 determinant, the final mesh can be generated using Display Tree $\rightarrow$ Premesh $\rightarrow$ Convert to Unstruct Mesh. The mesh generated using the steps described up to this point results in 1,879,920 nodes and 1,848,175 hexahedral elements. The export format can be set by using Output $\rightarrow$ Select Solver $\rightarrow$ Ouput Solver. There are over 100 output solvers that may be selected here. For the research presented in this thesis ANSYS CFX ${ }^{\circledR}$ is used as the fluid solver, so the ANSYS CFX option is selected. Next, the Output $\rightarrow$ Write Input option is selected which causes a window to pop up with additional options such as mesh scaling. The export file location is selected and the file is written. 


\section{Appendix B}

\section{CFX Pre Instructions}

ANSYS CFX ${ }^{\circledR}$ uses three separate programs during a complete CFD solution. It uses a pre-solver utility to set up the simulation, a solver utility to partition, run, and monitor the solution, and a post-processor to analyze the completed solution. The program called CFX-Pre is used first to define the problem. After beginning a new simulation, the previously constructed mesh can be imported by using File $\rightarrow$ Import Mesh. The domain size and number of elements can be checked be selecting Outline $\rightarrow$ Mesh $\rightarrow$ Mesh Statistics to make sure the correct mesh has been imported.

\section{B.1 Simulation Setup}

The first step is to set the simulation type by selecting Insert $\rightarrow$ Simulation Type. A new tab will appear allowing the basic settings for the simulation type to be set. For now the External Solver Coupling is set to None. If the two-way coupling method is to be used than this field will need to be modified and will be explained further in the coupling chapter. Simulation Type is set to Transient because the temporally varying pressure field is important. The LES method can only be used in transient type simulations. Time Duration is set to Number of Timesteps per Run, and Num. of Timesteps is set to $\mathbf{5 0 0 0}$. This setting is used to allow the flow to progress to a fully 
developed turbulent condition. Approximately 5000 timesteps is sufficient to successfully achieve a fully developed condition as determined by monitoring wall shear stress during the solution. The Time Steps field is set to Timesteps and then Timesteps is set at $\mathbf{0 . 0 0 1} \mathrm{s}$. This gives an average Courant Number of about 0.6 for $1 \mathrm{~m} / \mathrm{s}$ flow. A Courant Number between 0.5 and 1.0 is suggested for LES simulations [19]. Using a higher value causes excess damping of the turbulence, while values lower than this take unnecessary extra time steps to achieve a converged solution. The Initial Time field is set to Automatic with Value and Time is set to $\mathbf{0} \mathbf{s}$. This final option allows the initial time to be adjusted to automatically match the last time step from a previous run if the simulation is being restarted.

\section{B.2 Boundary Conditions}

The next step to setting up the simulation is to define the boundaries and specify conditions at those surfaces. Boundaries are created by using Insert $\rightarrow$ Boundary Condition, which will then bring up a prompt allowing the boundary to be named. The first boundary is named "Wall” and a details tab appears next to Outline titled Boundary: Wall. Choosing the boundary location is straight forward because of the part names selected when creating the mesh. Under Boundary: Wall $\rightarrow$ Basic Settings $\rightarrow$ Boundary Type the Wall option is selected. Location is also set to Wall. Under Boundary: Wall $\rightarrow$ Boundary Details both the No Slip and Smooth Wall options are selected. The $\mathbf{O k}$ button is then selected to save the settings for the wall boundary.

The inlet and outlet are set as a periodic boundary, which allows the flow to continue back in the inlet as it is exiting the outlet. This condition simulates a long pipe where the 
flow is allowed to reach a fully-developed state. The periodic condition is specified by first creating a domain interface by using Insert $\rightarrow$ Domain Interface. After selecting a name for the interface ("Domain Interface 1" is the default name and is used here) a new tab appears next to Outline which contains the basic settings for the interface. The first field, Interface Type, is set to Fluid Fluid. The domain interface contains two fluid surfaces called “Domain Interface Side 1” and Domain Interface Side 2.” Region List for Side 1 is set to Inlet, and region list for Side 2 is set to Outlet. This identifies the inlet and outlet as a common boundary where the flow properties will be matched. The Interface Models option is set to Translational Periodicity to indicate straight flow through the pipe. The Mass And Momentum box is checked to allow the mass flow rate through the interface to be specified. For water with an average flow velocity of $1 \mathrm{~m} / \mathrm{s}$ the mass flow rate is set to $8.068 \mathrm{~kg} / \mathrm{s}$. The $\mathbf{O k}$ button is selected to save the domain interface settings. The appearance of curved arrows at the pipe ends as seen in Figure B-1 indicates the successful creation of the periodic interface. 


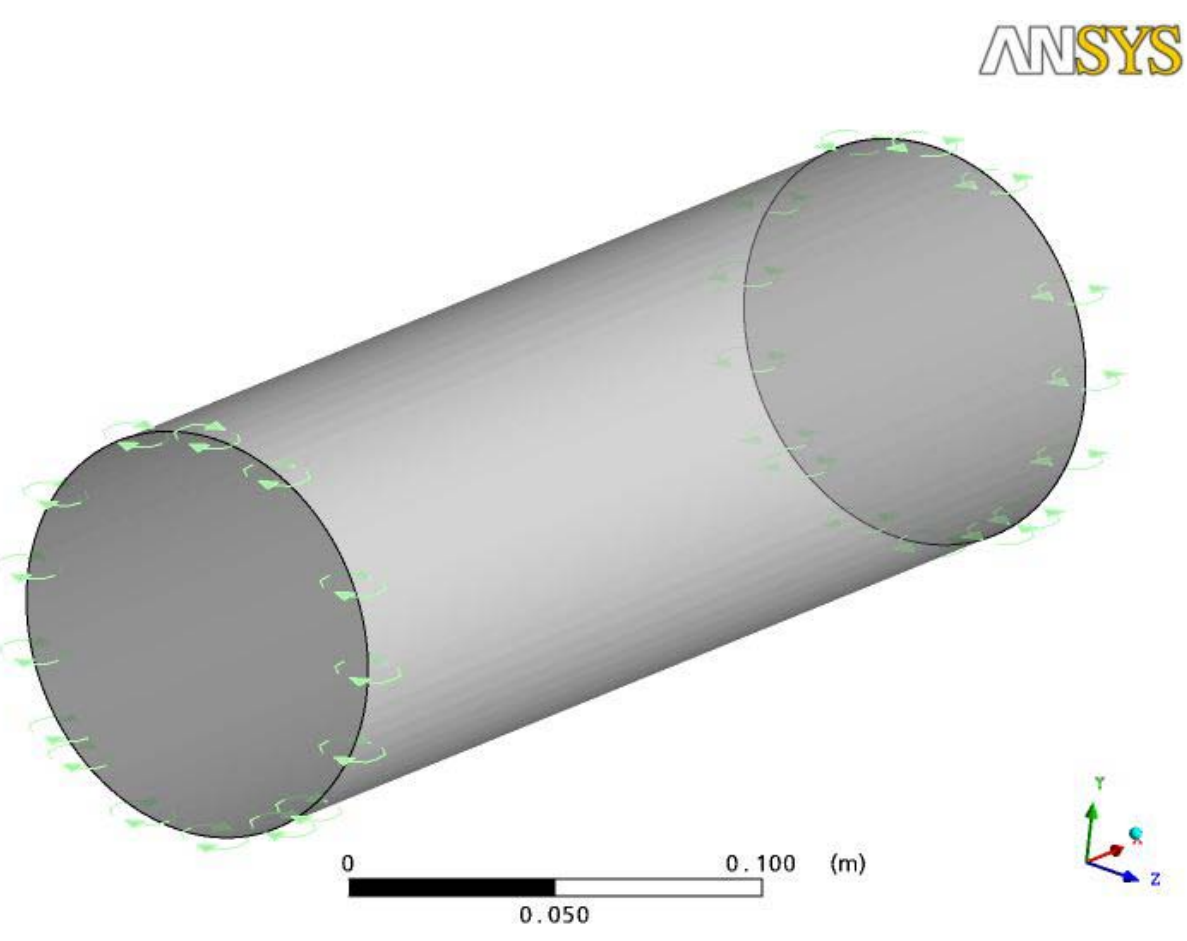

Figure B-1 CFX-Pre fluid domain showing periodic boundary

\section{B.3 Fluid Domain}

In order to define the conditions and settings for the fluid domain, a material must be specified. $\mathrm{CFX}^{\circledR}$ does include some predefined materials, but a user specified material allows more flexibility. The basic set of simulations for obtaining varying Reynolds Number all use water as the working fluid and vary the flow velocity. Verification of the non-dimensional solution, however, required modifying certain fluid properties independently of all others. A user material is defined by selecting Insert $\rightarrow$ Material and then choosing a name for the material (the default for the first new user material is “Material 1"). Under the Basic Settings tab for the material a brief description can be inserted, the material phase can be specified, and complex options can be set for reacting flows and mixtures. For these simulations the Option field is left as Pure Substance. Under the Material Properties tab the Option field is left as General Material to allow 
the user to specify all of the properties directly. The material properties tab has a section titled Thermodynamic Properties. In this section, leaving the Equation of State $\rightarrow$ Option field set to Value allows constant properties to be specified. For this research compressibility effects are not considered, so using a constant density fluid is appropriate. The Density field is set at $997 \mathbf{~} \mathbf{g g} / \mathbf{m}^{3}$ for most of the simulations, but can be varied to verify the non-dimensional solution. The Molar Mass field is not used because the flow is not reacting. Specific Heat Capacity is also not used because heat transfer is not considered important in these simulations and again, compressibility effects are ignored. The Reference State and Table Generation fields under Material Properties $\rightarrow$ Thermodynamic Properties are not used. Clicking the plus sign next to Material Properties $\rightarrow$ Transport Properties will expand the field to include additional options. A check mark must be placed next to Dynamic Viscosity to allow editing of the field. The Option portion is set to Value to use a constant viscosity. The Dynamic Viscosity portion of the field is then set for most simulations at $\mathbf{0 . 0 0 0 8 8 9 9} \mathbf{N} \cdot \mathbf{s} / \mathbf{m}^{2}$ but, like other properties, can be modified. The Thermal Conductivity property is not set and the remaining two fields, Radiation Properties and Buoyancy Properties, do not need to be expanded. The $\mathbf{O k}$ button is finally selected to save the changes and close the Material Properties tab.

After specifying the settings for the new material, the fluid domain can be set up by selecting Insert $\rightarrow$ Domain. This will bring up a prompt to name the domain (the name "fluid" is used for simplicity and clarity) and a new tab titled Domain: fluid. This tab will have three sub-tabs, General Options, Fluid Models, and Initialization. First, under Domain: fluid $\rightarrow$ General Options $\rightarrow$ Basic Settings, the Location field needs to 
be set to the named interior portion of the mesh ("fluid interior" was used when creating the mesh). Domain Type is set to Fluid Domain, and the user created material, Material 1, is selected under Fluids List. The default coordinate frame, Coord 0, is left selected under Coord Frame (this is the same Cartesian frame used for the mesh). The last Basic Settings field is for adding solid particles or bubbles to the fluid. No particles are used in these simulations so Particle Tracking is left turned off. Domain: fluid $\rightarrow$ General Options $\rightarrow$ Domain Models requires a fixed pressure to use as a reference. All pressures determined in the solution will be gauge pressures relative to this reference. Reference Pressure is set to $\mathbf{1 0 1} \mathbf{~ k P a}$. The other options under Domain Models are not used unless a non-inertial frame is being considered.

The next settings for the fluid domain are specified under the sub-tab Domain: fluid $\rightarrow$ Fluid Models. Because heat transfer is not being considered, the Heat Transfer $\rightarrow$ Option can be set to None and will reduce computation time. Turbulence $\rightarrow$ Option is set to LES Smagorinsky, which will greatly increase computation time, but is required in order to resolve the pressure fluctuations characteristic of turbulent flow. Turbulence $\rightarrow$ Wall Function is set to Automatic. This will let the solver decide whether to use scalable wall functions depending on the fineness of the mesh near the wall and the dimensionless wall shear stress. The plus sign by Turbulence $\rightarrow$ Advanced Control needs to be clicked to expand additional options. The Smagorinsky closure model for LES requires a single parameter called the Smagorinsky constant which is used to determine the sub grid scale (SGS) viscosity. The value of Turbulence $\rightarrow$ Advanced Control $\rightarrow$ Smagorinsky Model Constant is therefore set to $\mathbf{0 . 1}$ after checking the box 
next to this field. The Reaction or Combustion and Thermal Radiation Model are left set at None.

The final sub-tab under the fluid domain settings is Domain: fluid $\rightarrow$

Initialization. Because LES modeled flows are time accurate they require proper initial conditions. The flow field for these simulations is allowed to develop to a statistically steady state where statistical values are independent of the initial conditions, but the flow must be disturbed to allow turbulent structures to develop. This is done by initializing the flow field with random velocity fluctuations superposed on a uniform profile. The Domain Initialization box must be checked to make new options available. Velocity Type is left set to Cartesian, and Cartesian Velocity Components $\rightarrow$ Option is set to Automatic With Value. The $\mathbf{U}$ is set to $\mathbf{1}$, while $\mathbf{V}$ and $\mathbf{W}$ are set to $\mathbf{0}$. The Cartesian Velocity Components $\rightarrow$ Velocity Fluctuation box needs to be checked to add perturbations and Velocity Fluctuation is set to 0.1. Finally Static Pressure $\rightarrow$ Relative Pressure is set to $\mathbf{0}$ and the $\mathbf{O k}$ button is clicked to save changes. 


\section{Appendix C}

\section{CFX-Post Pressure Export Script}

After a fluid simulation is complete, with the pressure saved at each time step, the modified pressure needs to be calculated and exported for use in the structural simulations. Below is a CFX Command Language (CCL) code that can be used for this exporting step. It is used by saving the code in a text file with the extension '.cse'. The lines dealing with the export folder location must be updated to reflect the location where the comma delimited data files will be exported to. CFX-Post must be open and the results file from the fluid simulation must be loaded. Finally, the CCL code can be executed by choosing Session $\rightarrow$ Play Session, and selecting the previously saved .cse file.

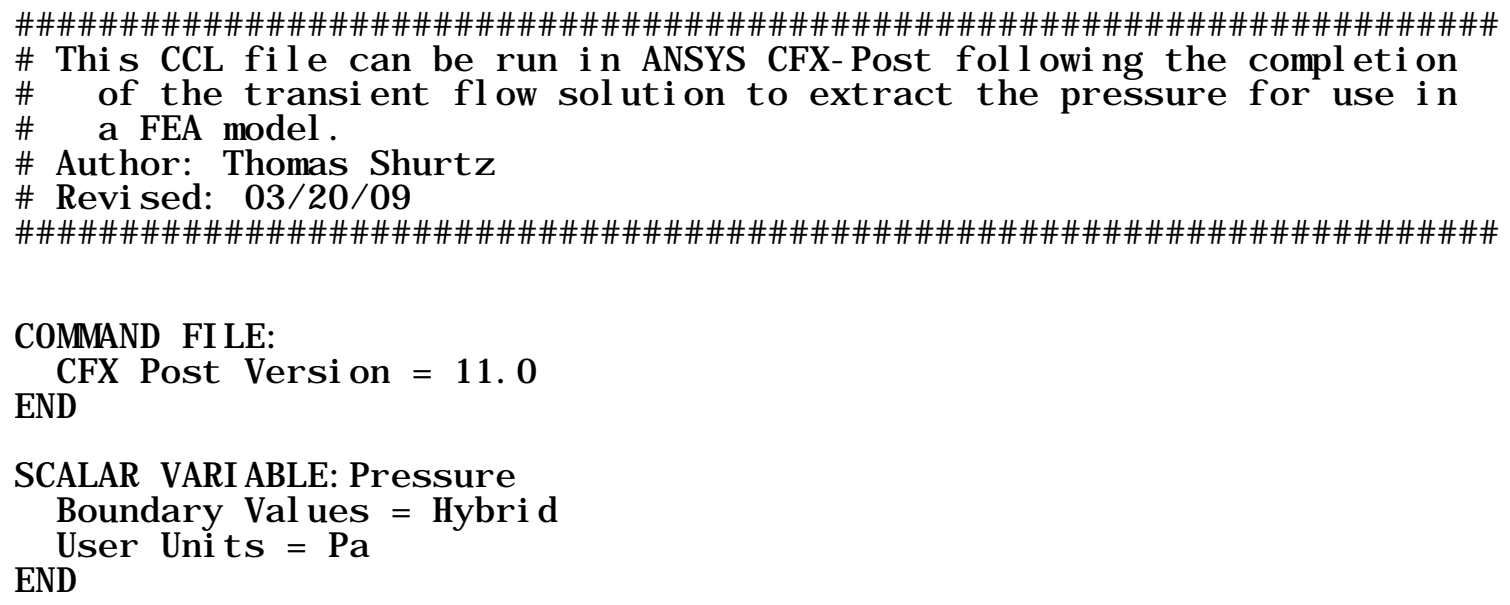




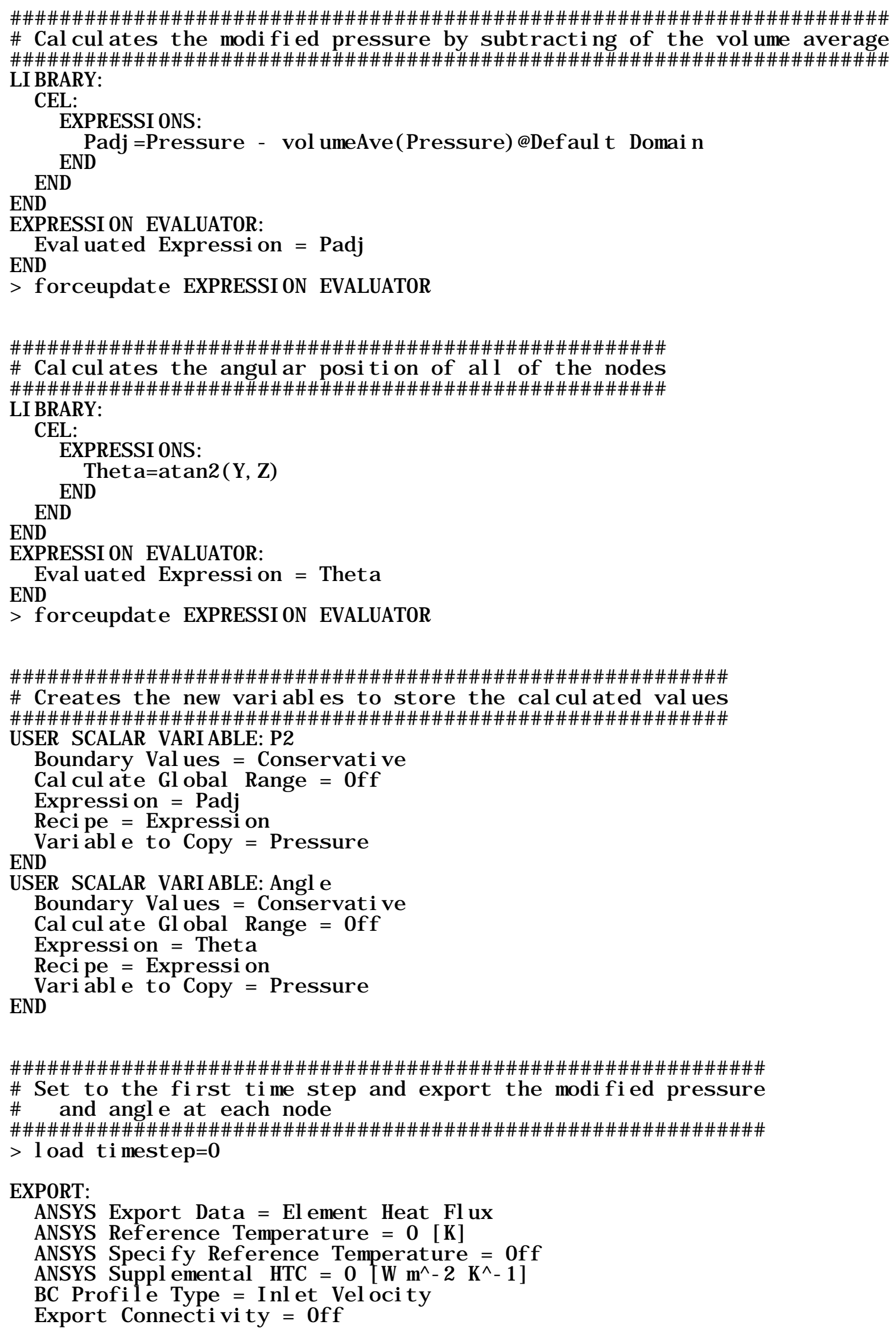


Export Coord Frame $=\mathrm{Gl}$ obal

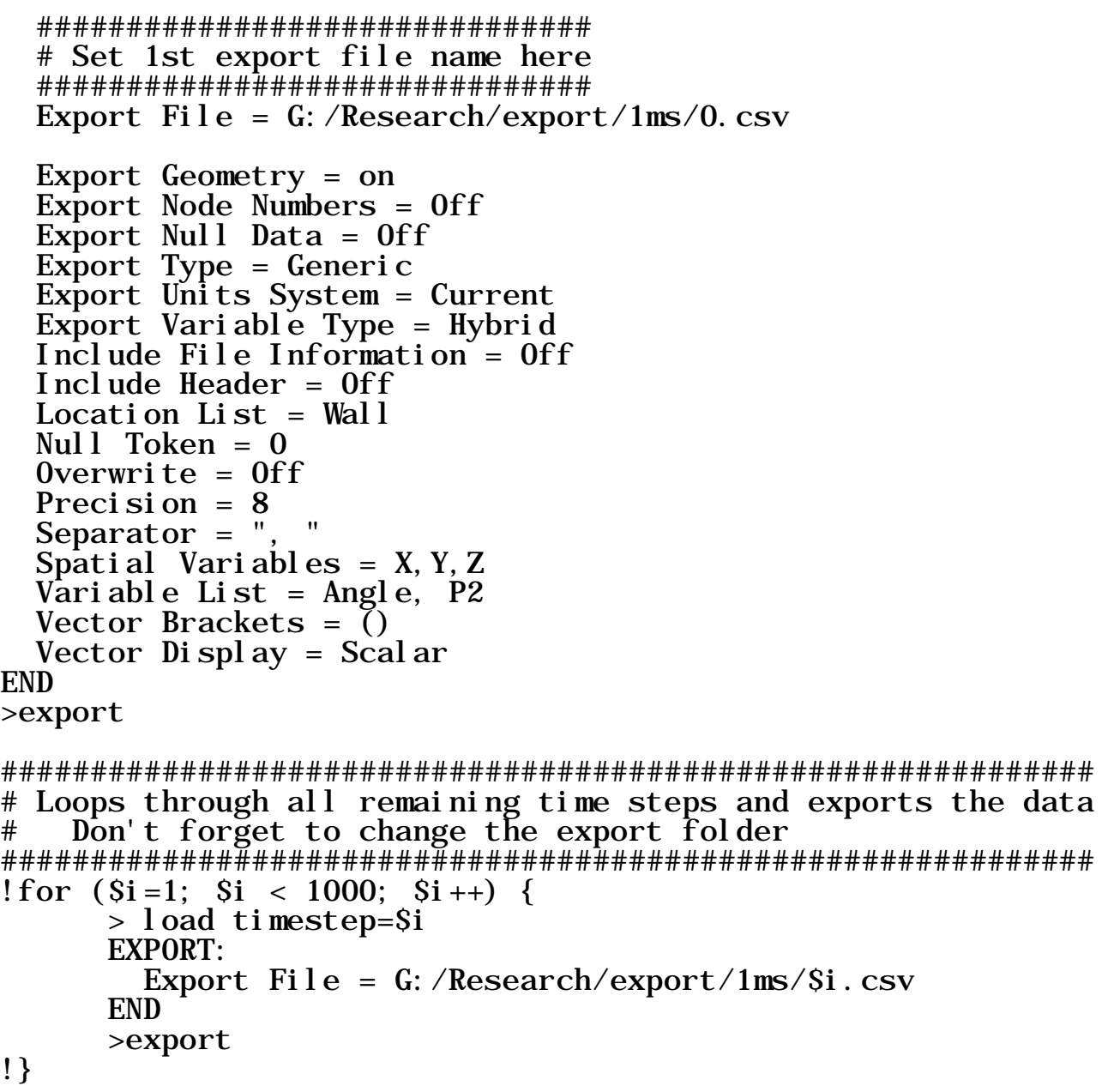




\section{Appendix D}

\section{Matlab Code for Pressure Mapping}

The modified pressure data from the comma delimited files must be reduced to match the structural mesh node locations. This Matlab code was written to import the pressure data time step by time step and write a corresponding load file for each one. The load step files can be imported into ANSYS during the structural simulations.

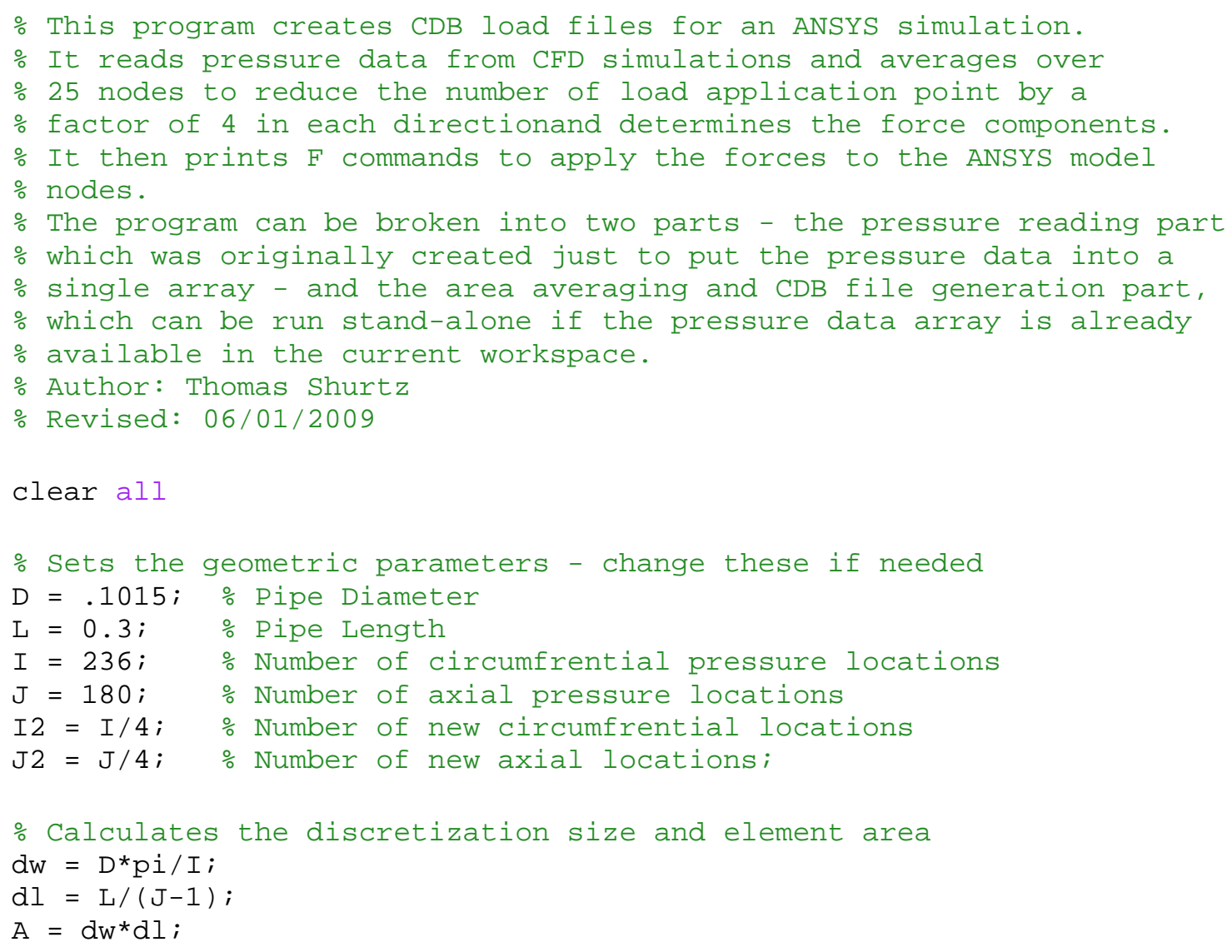


\% This loop goes through each time step and extracts the pressure

first $=0 ; \quad \%$ First file number in case offset is needed in file naming for $k=$ first:first +999

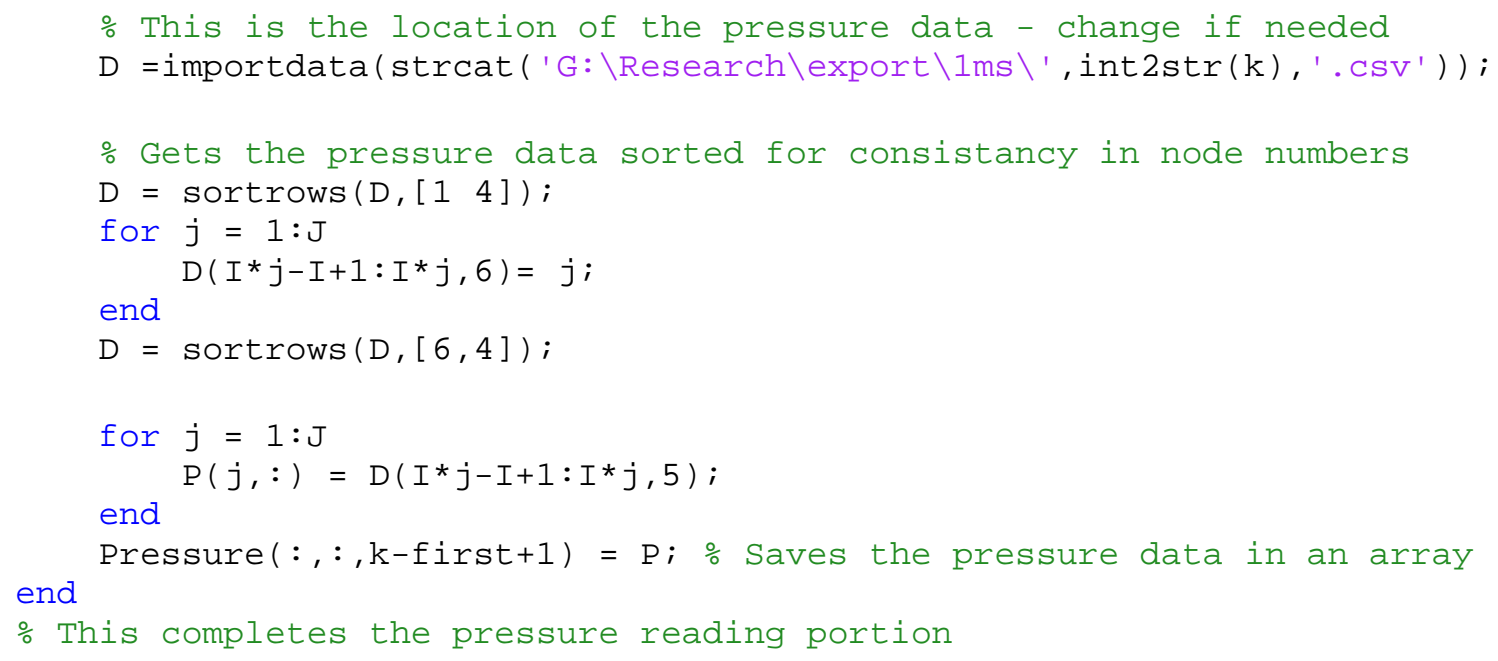




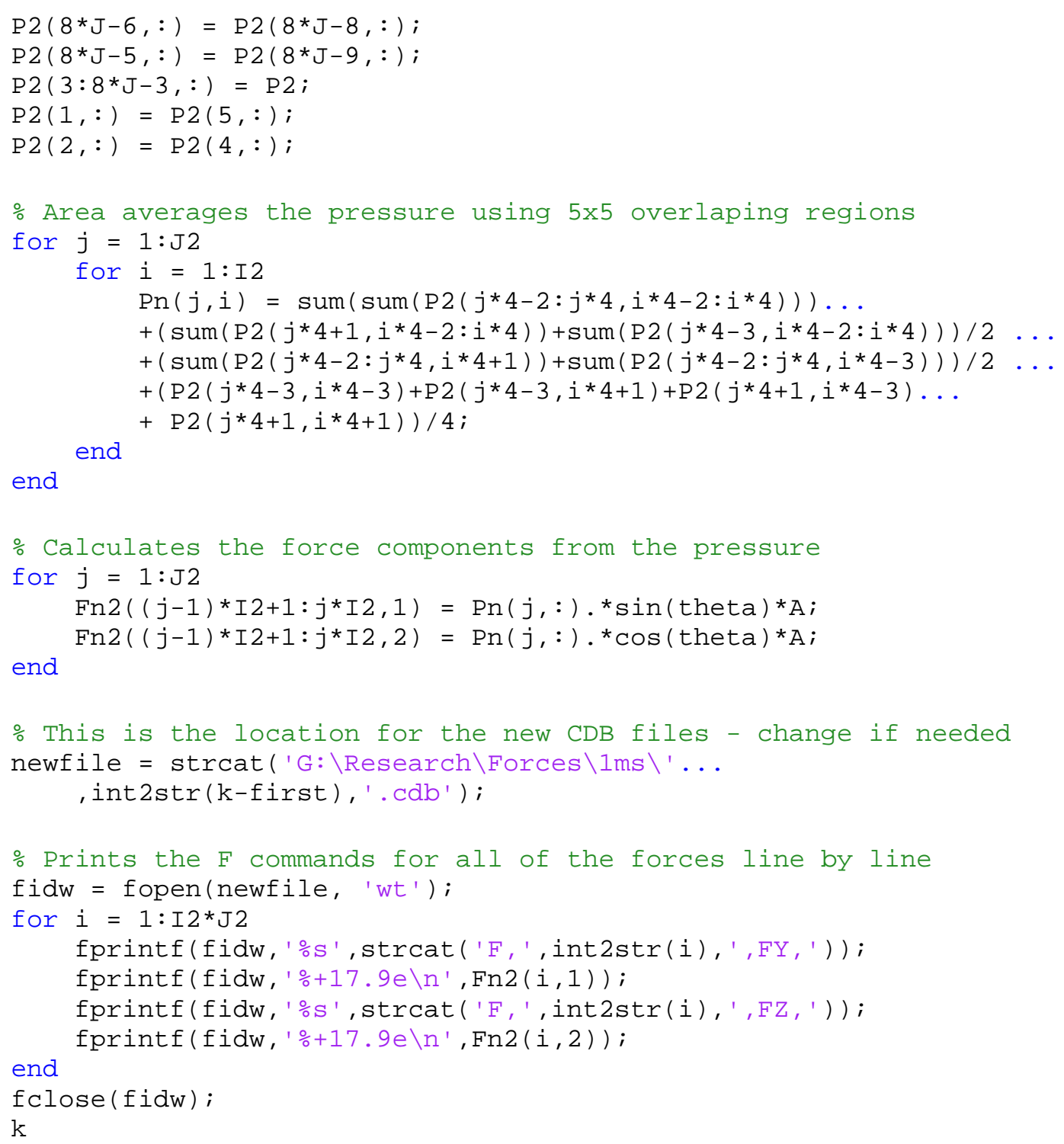

end 


\section{Appendix E}

\section{Matlab Code for Creating Nodes and Elements for Structural Model}

Because the CDB load files apply the forces at numbered nodes, it is important for the node numbering of the structural model to be consistent. This Matlab code is used to write a file that, when imported by ANSYS, will create the pipe geometry and elements with node numbers ordered correctly to accept loads from the CDB files created using the code in Appendix D. The file generated by this code is intended to be imported using the ANSYS batch file in Appendix F.

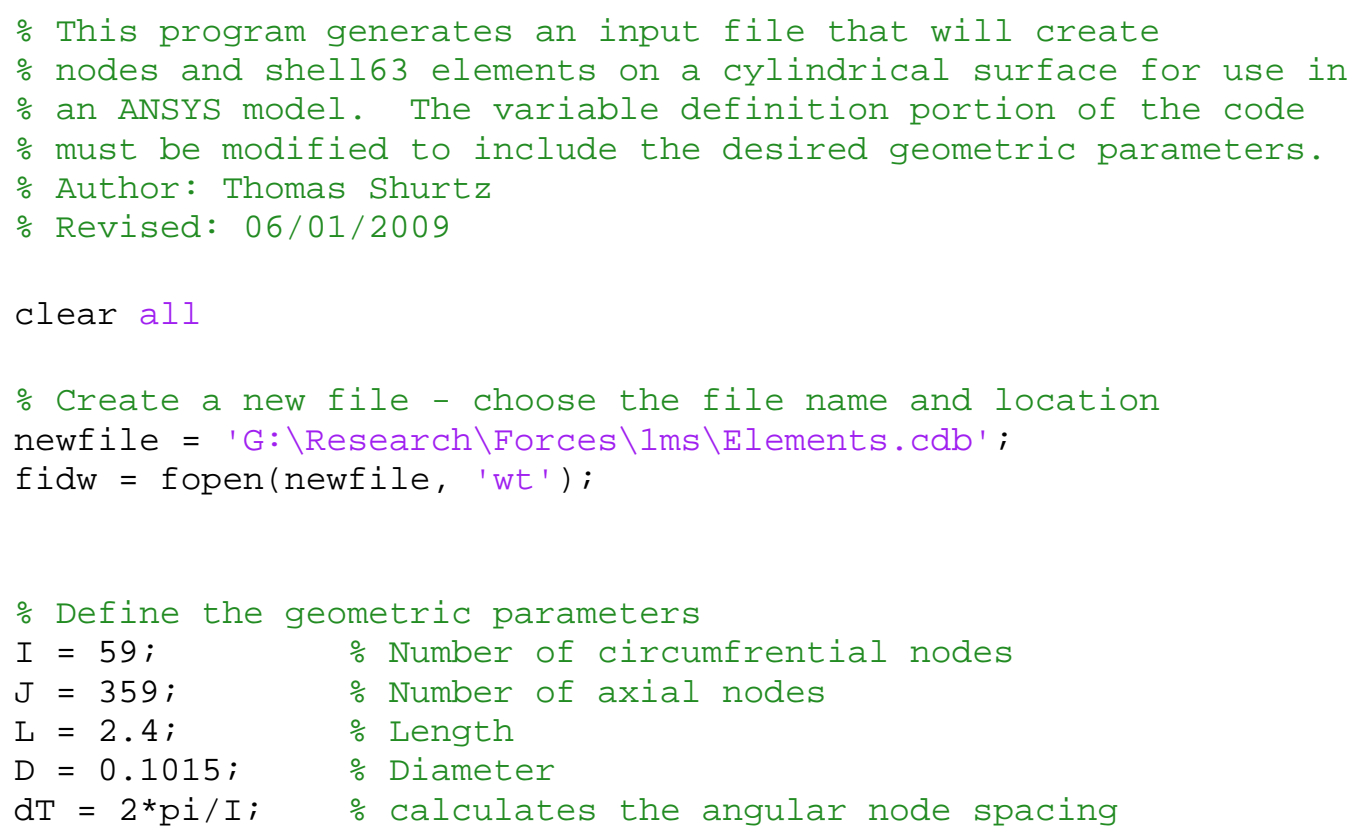




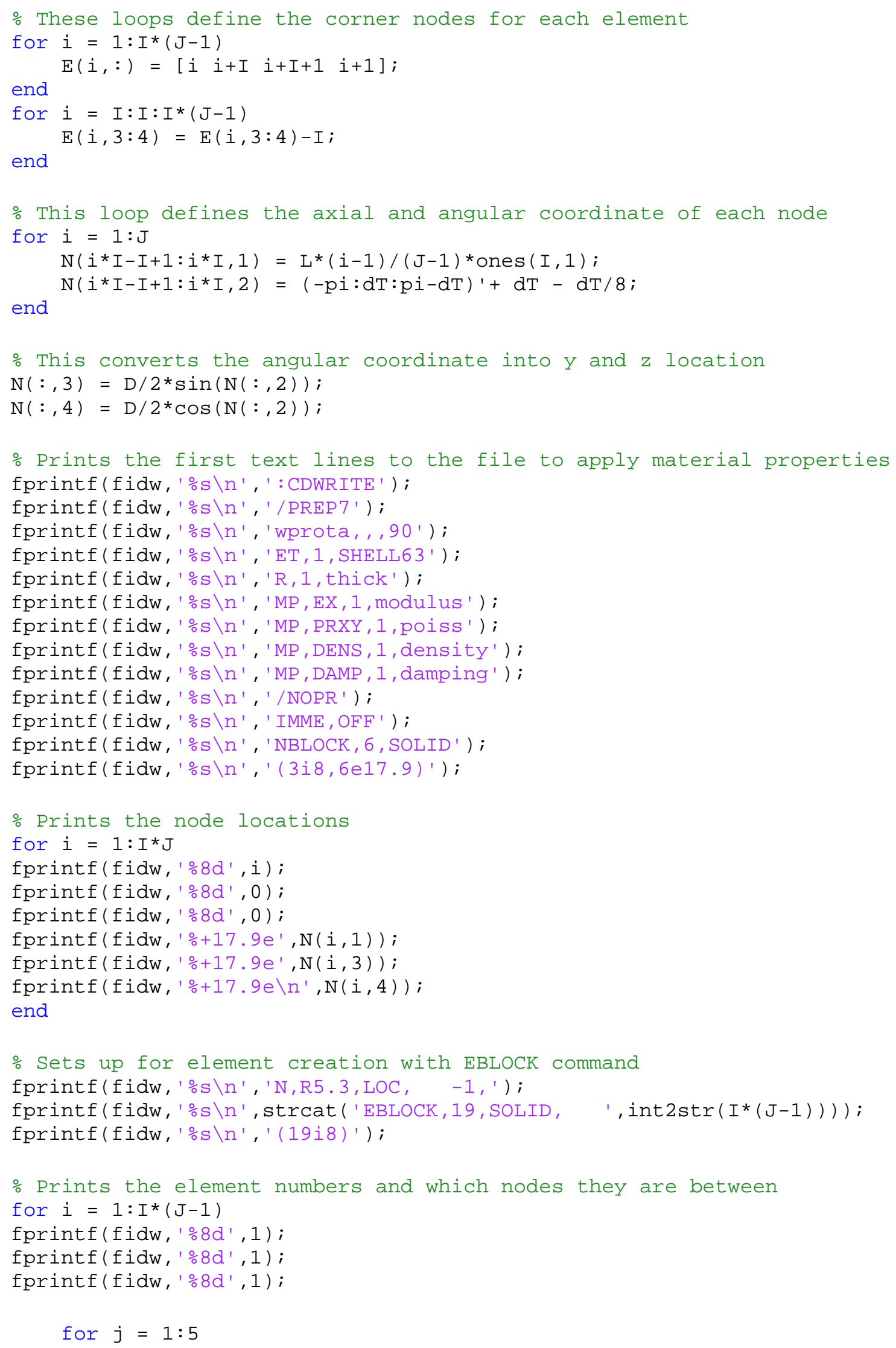




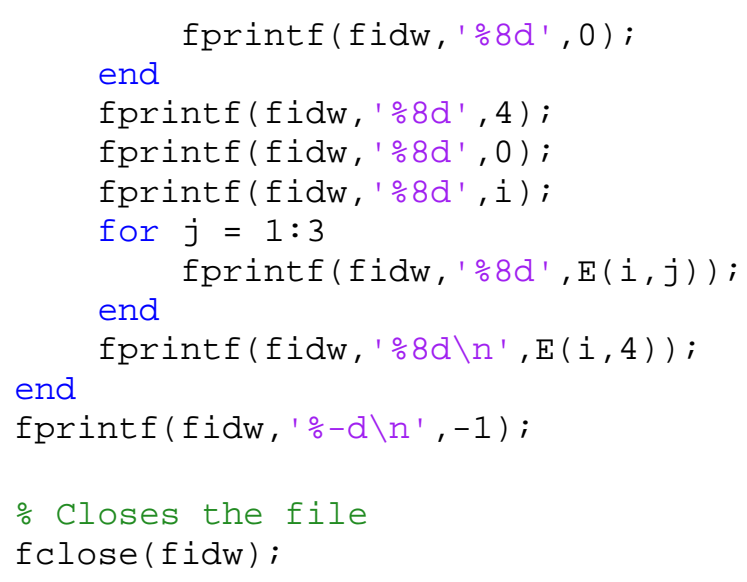




\section{Appendix F}

\section{ANSYS Batch File Used to Define and Run Structural Simulations}

The structural simulations require the solver settings to be specified and the CDB

file that creates the elements to be imported. They also require the changing load files to be imported each time step to update the fluid pressure induced loading. This batch file can be used as a template to define and run structural simulations.

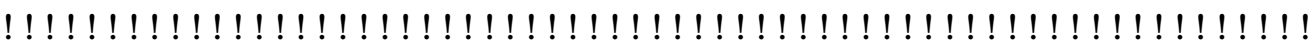

! Thi s batch file is used to set up and run ANSYS transient

! si mul ations for the l ong pi pe model. Onl y mi nor modification

! is requi red to make it sui table for the short pi pe nodel

! Aut hor: Thomas Shurtz

! Revi sed: 06/ 01/2009

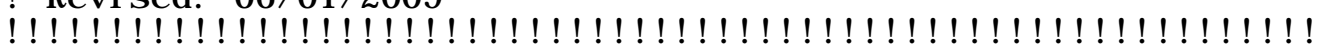

! Def i ne Parameters

*SET, or , . 05075

*SET, h, 2. 4

*SET, thi ck, 0.002

*SET, densi ty, 3000

*SET, modul us, 70000000000

*SET, poi ss, 0.3

*SET, dampi ng, . 001

*SET, L, 2. 4

*SET, TS, . 0001

! Pi pe I nsi de Di amet er

! Pi pe Lengt $h$

Pi pe Wal I Thi ckness

Pi pe Density

! Pi pe El asti c Modul us

! Pol sson's Rati o

! Materi al Damping Coef fi ci ent

! Cl ampi ng Di st ance

! Ti me step size

! I mport the el ement file to create the nodes and el ements

/ i nput, G: \Resear ch\For ces \El ement s, cdb
} 
! Set the boundary conditi ons (Adi ti onal fixed nodes mist be ! added for reduced $\mathrm{cl}$ ampi ng l engt h si mul at i ons)

d, 15, ux, 0

d, 15, uy, 0

d, 15, uz, 0

d, 44, uz, 0

d, 21078, uy, 0

d, 21078, uz, 0

! I mport the first I oad file

/ NOPR

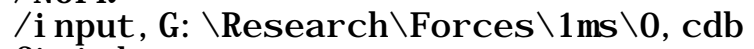

f i ni sh

! Enter the solver and specify the settings

! The first step is solved as steady to get i nitial position

/ sol u

t i me, TS

del $\mathrm{ti} \mathrm{mTS} / 2$

ant ype, t $r$ ans

ti m nt, of $f$, al I

rescont $r o l$, def i ne, none

$\mathrm{kbc}, 1$

nsubst, 2

OUTRES, er ase

OUTRES, al I, none

OUTRES, svar, none

OUTRES, I oci , none

OUTRES, NSOL, I ast

OUTRES, V, I ast

OUTRES, A, I ast

solve

! Turn timintegration on for al l other ti me steps

t i mi nt, on, al I

nsubst, 1

! Loop through each ti me step, updating forces at each one

$* D, j \mathrm{j}, 2,1000$

f del e, al I

/ NOPR

/ i nput, G: \Resear ch For ces $\backslash 1 \mathrm{~m} \backslash \mathrm{m} / \mathrm{j}-1 \% \mathrm{cdb}$

t i me, . 001*( j j )

solve

save

* ENDDO

fi ni sh 